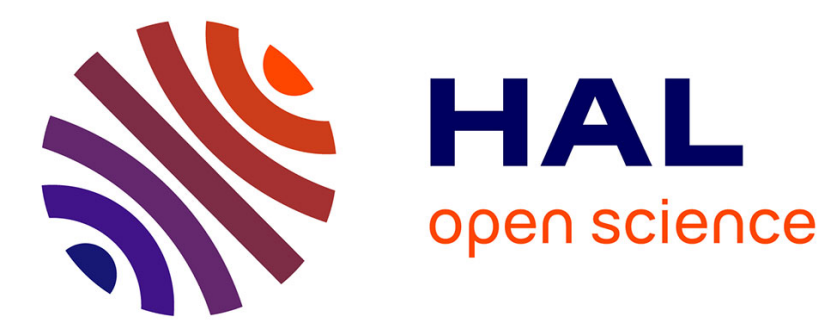

\title{
Impact-Seismic Investigations of the InSight Mission
}

Ingrid Daubar, Philippe Lognonné, Nicholas A. Teanby, Katarina Miljkovic, Jennifer Stevanović, Jeremie Vaubaillon, Balthasar Kenda, Taichi Kawamura, John Clinton, Antoine Lucas, et al.

\section{- To cite this version:}

Ingrid Daubar, Philippe Lognonné, Nicholas A. Teanby, Katarina Miljkovic, Jennifer Stevanović, et al.. Impact-Seismic Investigations of the InSight Mission. Space Science Reviews, 2018, 214 (132), pp.1-68. 10.1007/s11214-018-0562-x . hal-01990992

\section{HAL Id: hal-01990992 https://hal.science/hal-01990992}

Submitted on 23 Jan 2019

HAL is a multi-disciplinary open access archive for the deposit and dissemination of scientific research documents, whether they are published or not. The documents may come from teaching and research institutions in France or abroad, or from public or private research centers.
L'archive ouverte pluridisciplinaire HAL, est destinée au dépôt et à la diffusion de documents scientifiques de niveau recherche, publiés ou non, émanant des établissements d'enseignement et de recherche français ou étrangers, des laboratoires publics ou privés. 


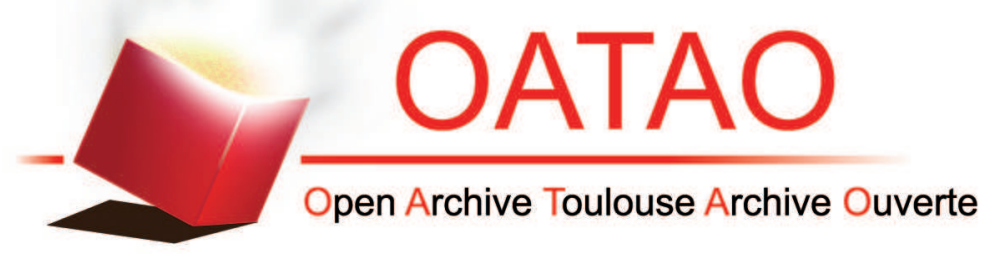

\section{Open Archive Toulouse Archive Ouverte (OATAO)}

OATAO is an open access repository that collects the work of some Toulouse researchers and makes it freely available over the web where possible.

This is an author's version published in: https://oatao.univ-toulouse.fr/21693

Official URL:https://doi.org/10.1007/s11214-018-0562-x

\section{To cite this version :}

Daubar, Ingrid and Lognonné, Philippe and Teanby, Nicholas A.,... [et al.] Impact-Seismic Investigations of the InSight Mission. (2018) Space Science Reviews, 214 (132). 1-68. ISSN 0038-630

Any correspondence concerning this service should be sent to the repository administrator: tech-oatao@listes-diff.inp-toulouse.fr 


\title{
Impact-Seismic Investigations of the InSight Mission
}

\author{
Ingrid Daubar ${ }^{1}$ - Philippe Lognonné ${ }^{2}$ Nicholas A. Teanby ${ }^{3}$ Katarina Miljkovic ${ }^{4}$. \\ Jennifer Stevanovićc $\hat{c}^{3,5}$ - Jeremie Vaubaillon ${ }^{6}$ - Balthasar Kenda ${ }^{2}$ - Taichi Kawamura ${ }^{7,2}$. \\ John Clinton ${ }^{8}$ - Antoine Lucas ${ }^{2}$ - Melanie Drilleau ${ }^{2}$. Charles Yana9 \\ Gareth S. Collins $^{10}$ • Don Banfield ${ }^{11}$ - Matthew Golombek ${ }^{1}$ - Sharon Kedar ${ }^{1}$. \\ Nicholas Schmerr ${ }^{12}$ - Raphael Garcia ${ }^{13}$ - Sebastien Rodriguez ${ }^{2}$ Tamara Gudkova ${ }^{14}$. \\ Stephane May ${ }^{9} \cdot$ Maria Banks $^{15}$ • Justin Maki ${ }^{1}$ Eleanor Sansom ${ }^{4}$. \\ Foivos Karakostas $^{2}$ - Mark Panning ${ }^{1}$ - Nobuaki Fuji ${ }^{2}$ James Wookey ${ }^{3}$. \\ Martin van Driel ${ }^{8}$ - Mark Lemmon ${ }^{16}$. Veronique Ansan ${ }^{17}$ - Maren Böse ${ }^{8}$. \\ Simon Stähler ${ }^{8}$ Hiroo Kanamori ${ }^{18}$ • James Richardson ${ }^{19}$ • Suzanne Smrekar ${ }^{1}$. \\ W. Bruce Banerdt ${ }^{1}$
}

Abstract Impact investigations will be an important aspect of the InSight mission. One of the scientific goals of the mission is a measurement of the current impact rate at Mars.

I. Daubar

ingrid.daubar@jpl.nasa.gov

1 Jet Propulsion Laboratory, California Institute of Technology, Pasadena, CA 91109, USA

2 Institut de Physique du Globe de Paris-Sorbone Paris Cité, Université Paris Sorbonne, Diderot, 75013 Paris, France

3 School of Earth Sciences, University of Bristol, Wills Memorial Building, Queens Road, Bristol, BS8 1RJ, UK

4 School of Earth and Planetary Science, Curtin University, GPO Box U1987, Perth, WA 6845, Australia

5 Atomic Weapons Establishment, Aldermaston, Berkshire, UK

6 IMCCE, Paris, France

7 National Astronomical Observatory of Japan, Tokyo, Japan

8 ETH Zurich, Zurich, Switzerland

9 Centre National d'Etudes Spatiales, Paris, France

10 Dept. Earth Science \& Engineering, Imperial College, London, UK

11420 Space Sciences, Cornell Center for Astrophysics and Planetary Science, Cornell University, Ithaca, MY 14853, USA

12 Department of Geology, University of Maryland, College Park, MD 20742, USA

13 ISAE-SUPAERO, Toulouse University, 10 Ave E. Belin, 31400 Toulouse, France 
Impacts will additionally inform the major goal of investigating the interior structure of Mars.

In this paper, we review the current state of knowledge about seismic signals from impacts on the Earth, Moon, and laboratory experiments. We describe the generalized physical models that can be used to explain these signals. A discussion of the appropriate source time function for impacts is presented, along with spectral characteristics including the cutoff frequency and its dependence on impact momentum. Estimates of the seismic efficiency (ratio between seismic and impact energies) vary widely. Our preferred value for the seismic efficiency at Mars is $5 \times 10^{-4}$, which we recommend using until we can measure it during the InSight mission, when seismic moments are not used directly. Effects of the material properties at the impact point and at the seismometer location are considered. We also discuss the processes by which airbursts and acoustic waves emanate from bolides, and the feasibility of detecting such signals.

We then consider the case of impacts on Mars. A review is given of the current knowledge of present-day cratering on Mars: the current impact rate, characteristics of those impactors such as velocity and directions, and the morphologies of the craters those impactors create. Several methods of scaling crater size to impact energy are presented. The Martian atmosphere, although thin, will cause fragmentation of impactors, with implications for the resulting seismic signals.

We also benchmark several different seismic modeling codes to be used in analysis of impact detections, and those codes are used to explore the seismic amplitude of impactinduced signals as a function of distance from the impact site. We predict a measurement of the current impact flux will be possible within the timeframe of the prime mission (one Mars year) with the detection of $\sim$ a few to several tens of impacts. However, the error bars on these predictions are large.

Specific to the InSight mission, we list discriminators of seismic signals from impacts that will be used to distinguish them from marsquakes. We describe the role of the InSight Impacts Science Theme Group during mission operations, including a plan for possible night-time meteor imaging. The impacts detected by these methods during the InSight mission will be used to improve interior structure models, measure the seismic efficiency, and calculate the size frequency distribution of current impacts.

Keywords InSight · Mars · Impact cratering · Seismology

\section{Introduction}

The Discovery mission InSight (Interior Exploration using Seismic Investigations, Geodesy and Heat Transport) (Banerdt et al. 2017) will study the interior of Mars using seismic signals. These will emanate from not only interior tectonic sources, but from impacts as well.

\footnotetext{
14 Schmidt Institute of Physics of the Earth RAS, Moscow, Russia

15 NASA Goddard Space Flight Center, Greenbelt, MD 20771, USA

16 Texas A\&M Univ., College Station, TX, USA

17 LPG Nantes, UMR6112, CNRS-Université de Nantes, 2 rue de la Houssinère, BP 92208, 44322 Nantes cédex 3, France

18 California Institute of Technology, Pasadena, CA, USA

19

Planetary Science Institute, Tucson, AZ, USA
} 
This paper describes the impact-related investigations being planned for the InSight mission, and how seismic detection of impact events will further the scientific goals of the mission.

The scientific goals of the InSight mission include both the direct measurement of impacts and other science that will benefit from the information impacts provide. Measuring the rate of crater formation at the surface will achieve the goal of determining the impact flux at Mars. Impacts will also inform the major goal of investigating the interior structure of Mars, as each impact will provide a set of seismic signals that have passed through the interior. Locating the corresponding craters precisely on the surface of the planet will provide a definitive source location, something that tectonic seismic sources will most likely not be able to accomplish because they are much less likely to have identifiable surface expressions. This additional information will inform seismic ray paths, seismic velocities, and the physical properties of the material through which the rays traveled.

The InSight seismometer, SEIS (Seismic Experiment for Interior Structure; Lognonné et al. 2018, this issue) is expected to record seismic signals from a number of impactors that regularly hit the Martian surface, and from these measurements estimate the rate of meteorite impacts on the surface of Mars. In addition, impacts could add a substantial number of seismic sources to an otherwise seismically quiet planet, whose natural quake rate is estimated to be $\sim 1000$ times lower than on Earth (Golombek et al. 1992; Golombek 2002; Knapmeyer et al. 2006; Plesa et al. 2018). See Lognonné and Mosser (1993), Lognonné and Johnson (2007, 2015), and Lorenz and Panning (2017) for comparisons of tectonically-driven seismicity and seismic detection perspectives.

The Impacts Science Theme Group (STG) was formed to oversee all of the impact cratering-related science of the InSight mission. Membership in the Impacts STG is open to any interested InSight science team member. The purpose of the group is to coordinate scientific analyses before and during the landed mission, and support operations to ensure the acquisition of impact-related data. Impact-related scientific analyses include the seismic source and waveform modeling of impact generated seismic signals; detection, localization, and characterization of impact sources; detection of meteors; modeling of meteor infrasound and acoustic source and shock signals; and comparative impact signal analyses between Mars, Earth and Moon.

In this paper, we summarize the current state of knowledge of impact-related seismology based on terrestrial and lunar studies, and the expectations for Martian impact seismology. The latter is based on our present understanding of the current impact rate and predictions of the Martian seismic response from the interior and atmosphere. We present a number of impact-seismic numerical models, benchmarked against each other in preparation for analysis of InSight data. Finally, Impacts STG operational and data analysis plans for the mission are also described.

\section{Background}

Impacts have been recorded seismically only on our own planet and the Moon. Without prior knowledge of what Martian impact-induced seismicity will look like, we must extrapolate from our knowledge of those two bodies to predict what InSight will observe on Mars.

\subsection{Impacts in Terrestrial Seismology}

Seismic signals from bolides were recognized as early as the beginning of the last century, with the detection of the seismic coupled airwave of the Tunguska event (Whipple 1930; 

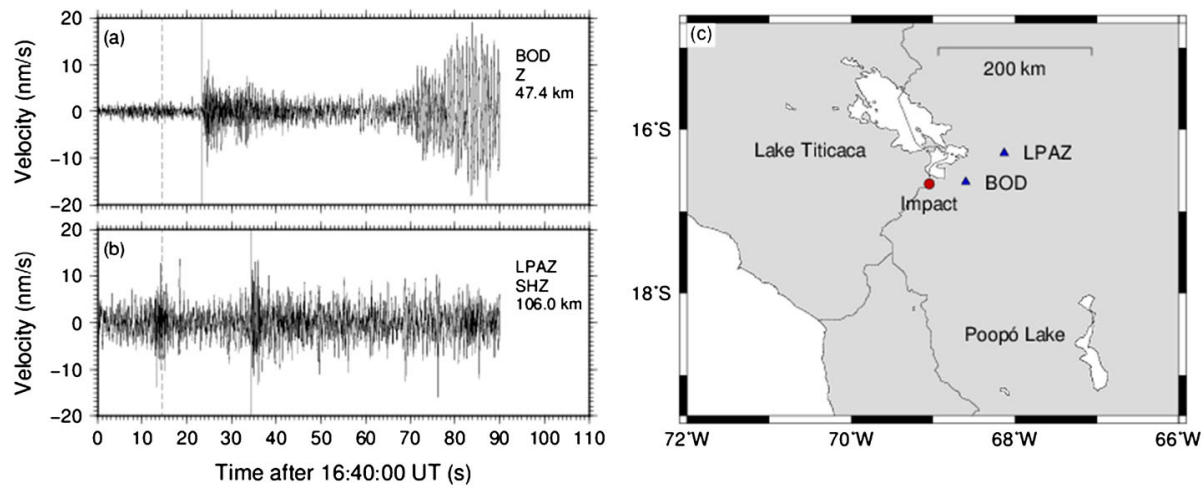

Fig. 1 Seismograms from the Carancas impact event in Bolivia. (a) Vertical seismogram from the closest station of the Bolivian Seismic Network. Dashed vertical line shows the origin time and solid vertical line shows the first arrival direct P-wave. The high-amplitude long-period signal at $70+$ seconds is the airwave. (b) Seismogram recorded at the LPAZ GSN station at $106 \mathrm{~km}$ offset. At this distance, the signal is already close to the ambient noise level. (c) Location map showing impact and station locations

Ben-Menahem 1975; Chyba et al. 1993). However, in general it is rare to detect seismic signals from meteoroid impacts on the Earth's surface, because its substantial atmosphere either ablates, fragments, or significantly slows the meteoroids before impact (Edwards et al. 2008). Most of the seismic signals detected from impacts are therefore associated with acoustic waves that have been converted to seismic waves at the Earth's surface. Earth is also farther from the asteroid belt than Mars, so has about half as many meteoroids of a given size impacting the top of the atmosphere (Davis 1993; Hartmann 2005; Williams et al. 2014), although the higher impact velocities at Earth balance this effect to some degree. This is in addition to the fact that the Earth is seismically very noisy, primarily due to oceanic, tectonic, atmospheric, and cultural noise sources (Peterson 1993). All these factors conspire to make detections of seismic waves from impact events extremely challenging on Earth.

A recent example of an impact that gave a detectable seismic signal was the Carancas event in Bolivia (Brown et al. 2008; Le Pichon et al. 2008; Tancredi et al. 2009), where an impact crater with a diameter of $13.5 \mathrm{~m}$ formed on 15 September 2007. This event had the advantage of being reported by eye witnesses, so the origin time is well constrained. There is some debate over the size and speed of the impactor, which may have had its velocity reduced by atmospheric drag from an original velocity of $10 \mathrm{~km} / \mathrm{s}$ to subsonic speeds of a few hundred meters per second by the time of impact. Adding to these complications, the impact was into water-saturated soil. Therefore, this impact may not be a particularly representative example of the kind of seismic signal we expect on Mars.

Figure 1 shows example seismograms from the Carancas impact recorded at distances of 47 and $106 \mathrm{~km}$. Because the signal is small, the event can only be seen at close distances. Hence, there is limited separation between phases, making identification and development of impact diagnostics difficult. Nevertheless, there is evidence of a reduced S-wave amplitude and a late-arriving airwave.

Airburst events are another potential source of seismic energy for InSight. An airburst occurs when a bolide enters a planetary atmosphere and abrubtly disrupts and decelerates, depositing much of its kinetic energy into a propagating acoustic wave in a manner similar to an explosion. This event is triggered when the dynamic pressure acting on the bolide as it traverses the planetary atmosphere exceeds the strength of the object. The precise altitude 

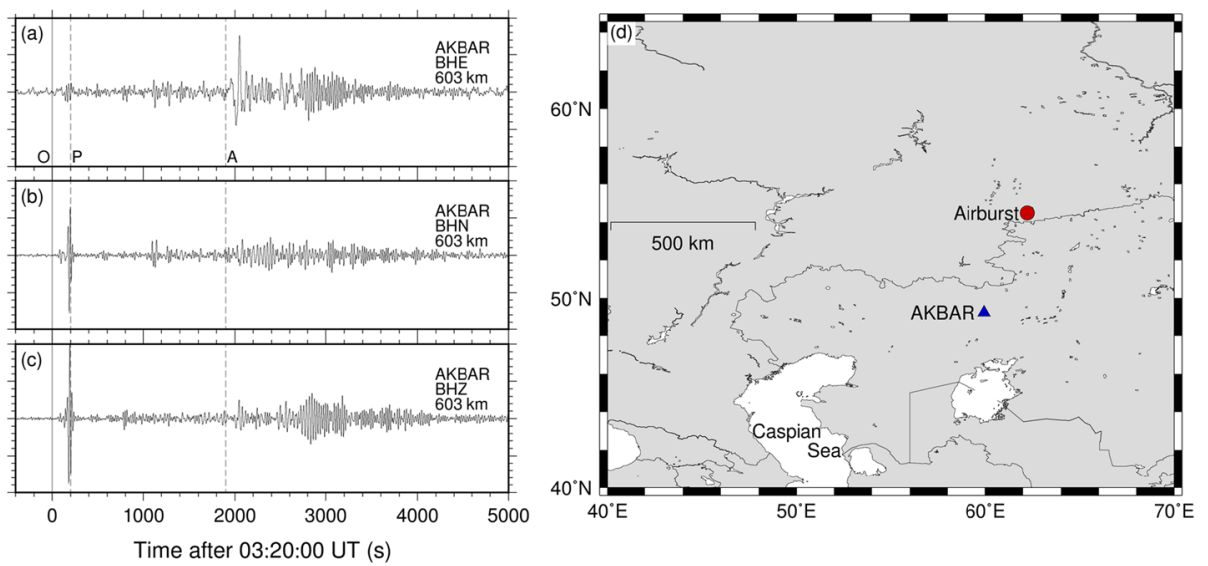

Fig. 2 Seismograms from the Chelyabinsk airburst event. (a, b, c) Three component seismograms recorded at AKBAR seismic station. Data have been filtered with a 20-200 s bandpass filter. Labelled vertical lines are: $\mathrm{O}$, origin time; $\mathrm{P}$, seismic precursor; and A, airwave arrival. The airwave is a low frequency wave, travelling at the speed of sound in air, with an emergent character. (d) Location map showing airburst event and station location

of disruption is governed partially by the material properties of the bolide and partially by the atmospheric density.

Airbursts are relatively common on Earth. The most notable recent event was the Chelyabinsk superbolide in 2013 over Russia (Brown et al. 2013), which was so large that acoustic energy coupled into the ground and was able to propagate as seismic energy (Fig. 2). Another notable example of an airburst that generated both seismic and acoustic detections was the Oregon State Bolide in 2008, which occurred directly over the US seismic array. It is expected that airbursts will be a significant source of both seismic and acoustic signals for InSight, given the larger impactor population and quieter environment (Brown et al. 2013; Stevanović et al. 2017). Section 4.5 discusses airburst events in detail, including detection plans with InSight.

\subsection{Impacts in Lunar Seismology}

The first extraterrestrial seismic observations were made on the Moon by the Apollo missions. The Apollo program performed almost eight years of seismic studies from 19691977, including five years of network observation with four seismic stations. During this time, more than 13000 events were identified. Among the detected seismic events, meteorite impacts were the second largest group; approximately 1800 impacts were identified (Nakamura 2003). On airless bodies such as the Moon, impactors fall directly on the ground and generate seismic signals. This is different from the Earth or Mars, where impactors first interact with the atmosphere. For a m-scale impactor, deceleration in the atmosphere can lead either to an airburst combined with possible subsonic surface impacts (for most terrestrial impacts), or to both an airburst and supersonic ground impacts (for Martian impacts). Impactors of this scale can also be entirely ablated in an atmospheric layer so that no fragments reach the ground. Thus, on planets and satellites with atmospheres, small meteoroids are potentially more detectable using acoustic airwaves than seismic waves, and only large impactors reach the surface. On the Moon, the lack of an atmosphere implies all impacts are detected through their ground displacement alone. 


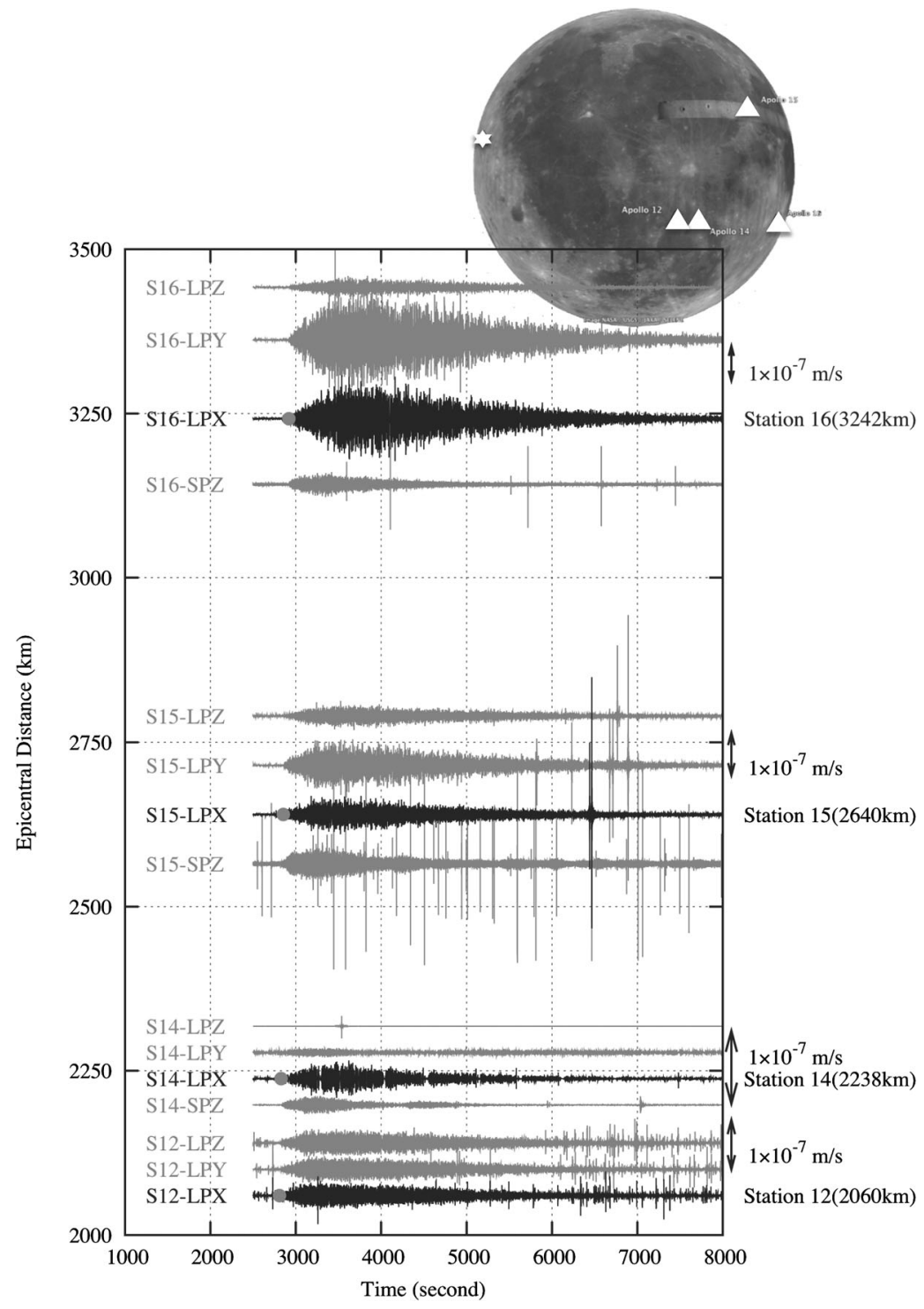

Fig. 3 Ground velocity records from the Apollo seismic network of a large natural impact occurring on November, 14, 1976. Black seismograms indicate the axes with the best signal to noise ratio, which were used for arrival time readings and seismic velocity models The mass of the impact has been estimated to about 25-35 tons assuming an impact velocity of $20 \mathrm{~km} / \mathrm{s}$ (Gudkova et al. 2011). The lunar globe (LROC images; http://photojournal.jpl.nasa.gov/catalog/PIA14011) shows locations of the Apollo seismic stations (white triangles) and of the impact (white star). Reprinted from Lognonné and Kawamura 2015. Note spikes are artifacts of Apollo data acquisition

Figure 3 shows an example of seismic events observed on the Moon recorded by the Apollo seismic network up to a distance of $3242 \mathrm{~km}$. Because impacts are superficial events, their signals propagate through the fractured megaregolith layer (brecciated material $1-3 \mathrm{~km}$ thick) and crust twice: once below the source, and then again below the detecting station. 

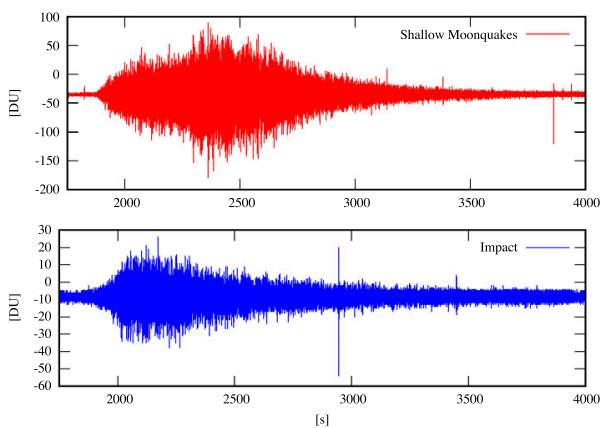

[s]

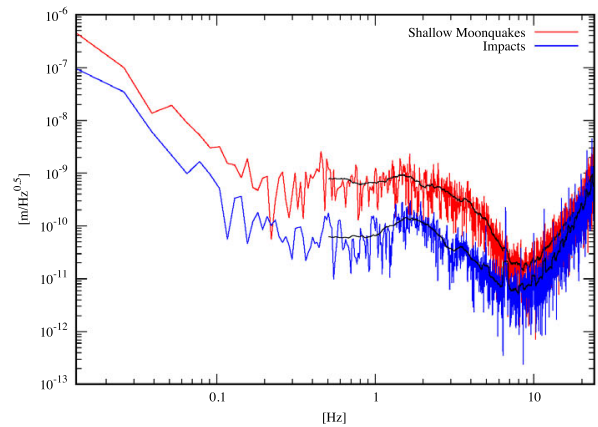

$[\mathrm{Hz}]$

Fig. 4 Comparison of waveforms and spectra from a lunar quake (red) and a lunar impact (blue). Smoothed spectra are also plotted for comparison (black and gray respectively). Both sets of data are from Apollo Station 16. The time series on the left are from the short period seismometer, and the spectra on the right are the combined spectra of long and short period seismic data. The shallow moonquake is from 1975/1/13/00:28 and the impact is from 1976/1/13/7:14

Lunar seismograms are thus characterized by intense scattering and resulting long, ringing coda (backscattering waves due to heterogeneities). The scattering mainly occurs in the megaregolith layer, which has been "gardened" by many impacts and as a result is highly porous and fractured. Thus impact signals experience more scattering compared to endogenic events such as deep and shallow moonquakes (Gudkova et al. 2011). The coda of lunar impacts are longer than that of deep and shallow moonquakes and may last for as long as an hour. Figure 4 shows an illustration of the difference between the spectra of an impact and a shallow moonquake, occurring at comparable distance. Clear differences in the waveform and the coda can be seen, and thus we can discriminate quakes from impacts (this will be discussed further in Sect. 6.1).

The relationship between seismic signals and impact energy was studied using artificial impacts. During the Apollo missions, the seismometers detected seismic signals generated by the lunar module ascent stage and Saturn IV B booster impacts (Latham et al. 1970a, 1970b; Toksöz et al. 1972). These impacts have known event times, locations, and impact energies, so they could be used to calibrate the relation between the impact energy and seismic energy. Recently, the Lunar Reconnaissance Orbiter Camera (LROC) imaged the actual craters of these artificial impacts in high resolution, which gives another constraint on crater size for a known impact energy (mass and velocity) (Wagner et al. 2017). It should be noted, however, that compared to natural impacts of asteroids or comets, these artificial impactors had very low average densities, low impact velocities, and in many cases highly oblique impact angles. For all of these reasons, the seismic signals produced by the booster impacts may not be representative of natural impacts, but they are some of the best (only) analogs available with known impact parameters.

On the Moon, natural impacts are all deduced based on seismic investigations. No crater thought to be responsible for specific seismically identified events has been detected to date, a task made nearly impossible by the extremely small fraction of the Moon covered by adequate Apollo orbital imaging and the large location estimate errors for these events (as much as tens of kilometers). Identification of exact source locations through images or other independent observations will thus be very helpful for the seismic investigations of InSight (see Sect. 7), and the first time this will be accomplished on another planet.

Presumed impact events with high signal to noise ratios have been located through travel time analyses using the Apollo seismic network. Other impacts with smaller signal to noise 
ratios were identified through analyses of coda features and epicentral distances. Out of the 1,800 events listed in the Nakamura catalogue, very few have been located. One of the largest collections is in Gudkova et al. (2015), with 40 locations. Even fewer natural impacts have been used for lunar structural inversions (14 in Khan and Mosegaard 2002; 19 in Lognonné et al. 2003; Chenet et al. 2006).

Despite these limitations, the analysis of the frequency-magnitude collection of seismically detected lunar impacts has been used to estimate the flux of meteorites in the EarthMoon system (Oberst and Nakamura 1989; Lognonné et al. 2009; Oberst et al. 2012). Those estimates were comparable to those obtained from other means.

Impacts have also provided key data for the determination of the lunar crustal thickness. Surprisingly, an impact provided the deepest direct seismic ray recorded by lunar seismometry (Nakamura et al. 1973). For determining the structure of the lunar crust, the best data are from artificial impacts, for which times and locations are known with high precision. This provided $\mathrm{P}$ and $\mathrm{S}$ travel times directly useful for structural inversions (Nakamura et al. 1976; Khan and Mosegaard 2002; Lognonné et al. 2003; Gagnepain-Beyneix et al. 2006; Lognonné and Johnson 2007, 2015; note corrections for timing problems made by Nakamura 2011). Natural impacts were also used for these inversions when more than three precise arrival times were measured on the Apollo network. They could also be used to derive estimates of the crustal thickness at the impact sites. Chenet et al. (2006) took advantage of this and carried out joint inversions with seismic and gravity data to construct a 3D crustal thickness map of the Moon.

\section{Seismic Signals from Impacts in General}

Seismic signals from impacts differ in several important ways from seismic signals from internal, tectonic sources. First, the source function for an impact is modeled better by a single source representing an explosive expansion from a point, rather than the doublecoupled force typical of a quake. This results in spectra with a different frequency content from an impact. Subsurface material properties have a larger effect in the case of impacts, because a source depth of essentially zero means the signal travels through the shallow subsurface twice, enhancing the effects of e.g. a porous or fractured upper layer. Finally, in the specific case of Mars with its thin atmosphere, atmospheric effects also must be taken into consideration.

Two different approaches have been developed by the community. The first one uses an equivalent source function of an impact, which can then be used for modeling of synthetic waveforms, in a way comparable to using seismic double couple equivalent forces for quake modeling. This force is generally characterized by its long period dependency and by the frequency cutoff, where that long period dependency breaks. The second approach is based on the seismic energy efficiency of an impact. This is related to the amplitude of the seismic waves and/or equivalent seismic moment of the source generating the waves. Here we present and compare these two approaches.

\subsection{Impact Seismic Equivalent Source Time Function}

An impact is a complex process during which some of the impactor's momentum and energy are transmitted to the target. For small impacts (impactors $<100 \mathrm{~m}$ diameter) on planets with a dense atmosphere, like Earth or Venus, almost all of the impactor's kinetic energy is deposited in the atmosphere. For planets lacking an atmosphere, the impactor hits the 

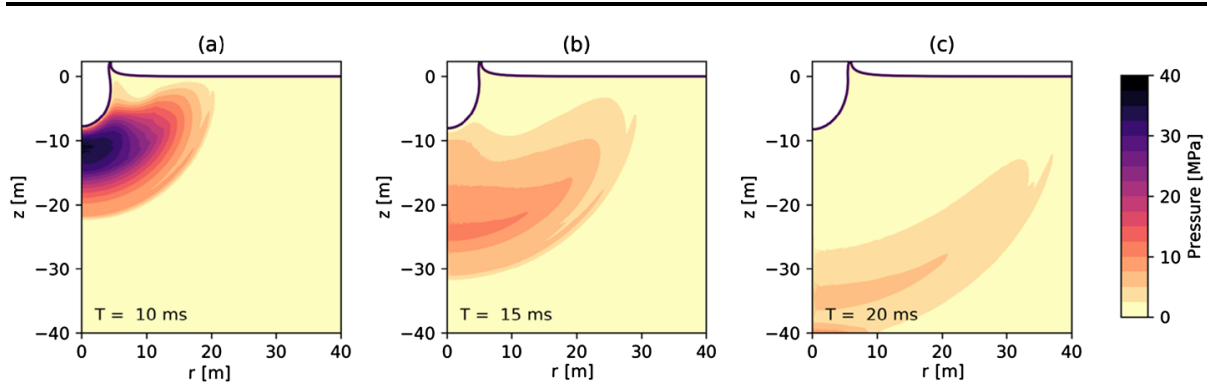

Fig. 5 An example of an iSALE-2D hydrodynamic simulation showing a 1-m radius basalt impactor striking Mars regolith at $7 \mathrm{~km} / \mathrm{s}$; snapshot $10 \mathrm{~ms}$ (a); $15 \mathrm{~ms}$ (b) and $20 \mathrm{~ms}$ (c) after the impact. Note the expansion of the hemispherical shock wave; this is the primary source of seismic signal. The interaction with the free surface is also visible via reduction in the shock pressure close to the surface

ground directly, where all the energy is released (for a general review of impacts in planetary seismology see Lognonné and Johnson 2007, 2015). Mars is intermediate, where the kinetic energy of meter-scale impactors will be released both in the atmosphere during the entry and passage, and on the ground at the final impact.

The seismic source, or source time function, $f(t, \mathbf{r})$, represents the associated force field acting on the planetary surface and subsurface during the impact process. Its mean amplitude will depend on the energy of the impact, and its time dependency will depend on the shock wave propagation time, during which the seismic energy is radiated. The impact source has been approximated using a variety of different methods, ranging from permanent volume injection (Richardson et al. 2005), full hydrodynamic simulations of particle motions and stress (Ivanov and Artemieva 2002), scaling laws derived from explosive and low-velocity impacts (Teanby and Wookey 2011), and as a momentum transfer (Lognonné et al. 2009; Gudkova et al. 2011, 2015). Models of seismic source time function for impacts proposed by Gudkova et al. $(2011,2015)$ followed analysis of lunar Apollo seismic data. Another model proposed by Shishkin (2007) is based on scaling laws and past nuclear explosion surface tests (e.g. Haskell 1967; Werth and Herbst 1963). All of these models are a simplified view of the shock wave propagation, which generates strength failure and plastic displacements during its strong regime, and nonlinear displacements during its semi-strong regime before it transitions into an elastic wave. Figure 5 shows a snapshot of such a shock wave for a numerical simulation of a 1-m radius impactor striking Mars regolith at an impact velocity of $7 \mathrm{~km} / \mathrm{s}, 10 \mathrm{~ms}$ to $20 \mathrm{~ms}$ after the impact.

Gudkova et al. $(2011,2015)$ (referred to hereafter as model GL) proposed that an impact signal is similar to the one generated by the release in a small shocked volume of a point force density:

$$
\mathbf{F}_{0}(t, \boldsymbol{x})=F_{0}(t) \delta_{3}\left(\boldsymbol{x}-\boldsymbol{x}_{s}\right) \operatorname{Sm} \mathbf{v} \frac{d g(t)}{d t},
$$

with

$$
\begin{aligned}
& F_{0}(t)=\operatorname{Sm} \mathbf{v} \frac{d g(t)}{d t}, \\
& g(t)=H\left(t+\tau_{1}\right) H\left(\tau_{1}-t\right)\left(1+\cos \left(\omega_{1} t\right)\right),
\end{aligned}
$$

where $m$ and $\mathbf{v}$ are the mass and velocity of the impactor, respectively, and $S$ is an amplification factor related to the ejecta given by Lognonné et al. (2009) as a function of the impact 
Table 1 Source models used in this analysis for a homogeneous medium. The second, third and fourth column are those of the impact models of Gudkova et al. (2011, 2015) (GL), Shishkin (2007) model updating Werth and Herbst (1963) (SWH), and a classical Seismic Moment tensor model (Aki and Richards 2002) (SM). For the SWH model, $V_{\infty}$ is the volume of the fractured part of the crater and can be estimated as $\frac{4 \pi}{3} \frac{\sigma_{S}}{\mu}\left(\frac{S_{0}}{\pi}\right)^{3 / 2}$. A dot indicates the derivative of the function

$$
\text { GL model SWH model SM model }
$$

\begin{tabular}{llll}
\hline $\begin{array}{l}\text { Far field } \\
\text { displacement }\end{array}$ & $u(r, t)=\frac{1}{4 \pi \rho v_{p}^{2}} \operatorname{Smv} \frac{\dot{g}\left(t-\frac{r}{v_{p}}\right)}{r}$ & $u(r, t)=\frac{1}{4 \pi} \frac{V_{\infty}}{v_{p} \tau_{0}} \frac{\dot{f}\left(\frac{t-r / v_{p}}{\tau_{0}}\right)}{r}$ & \\
$\begin{array}{llll}\text { Equivalent } \\
\text { moment }\end{array}$ & $v_{p} \operatorname{Smvg}(t)$ & $\rho v_{p}^{2} V_{\infty} f(t)$ & $M(t)=\frac{1}{4 \pi \rho v_{p}^{3}} \frac{\dot{M}\left(t-\frac{r}{v_{p b r}}\right)}{r}$ \\
Units & $\mathrm{m} / \mathrm{s} \mathrm{kg} \mathrm{m} / \mathrm{s}=\mathrm{Nm}$ & $\mathrm{kg} / \mathrm{m}^{3} \mathrm{~m}^{2} / \mathrm{s}^{2} \mathrm{~m}^{3}=\mathrm{Nm}$ & $\mathrm{Nm}$ \\
\hline
\end{tabular}

velocity. The source function $g(t)$ is a cosine function over half a period, $\omega_{1}=\pi / \tau_{1}$, and $H(t)$ is the Heaviside function. For an infinite medium, such a source leads to a far field displacement as in the second column of Table 1. For P waves, it has a seismic equivalent moment provided by:

$$
M(t)=v_{p} S \operatorname{mvg}(t),
$$

where $v_{p}$ is the seismic velocity of body waves in the vicinity of the impact location. The amplitude of the waves is proportional to the time derivative of this moment (Gudkova et al. 2015). Although matching the Apollo signal in the body waves bandwidth, this source representation is nevertheless not compatible with any static permanent deformation which could occur near the source location, as the mean of $g(t)$ cancels out.

Shishkin (2007), following Haskell (1967) and Werth and Herbst (1963), considered a source function without discontinuities for displacement, velocity and acceleration (referred to hereafter as the SWH model). The source function is defined as:

$$
f(\tau)=1-\exp (-\tau)\left(1+\tau+\tau^{2} / 2+\tau^{3} / 3-B \tau^{4}\right),
$$

where $\tau$ is a non-dimensional time, defined as $\tau=t / \tau_{0}$, where $\tau_{0}$ is the timescale of the shock wave, comparable to the $\alpha^{-1}$ parameter of the Rayleigh pulse model, and $B$ is a parameter that depends on the material properties of the medium. Such a source is a generalization of the one discussed later in Sect. 3.3. This leads to a displacement in an infinite medium as given in the third column of Table 1 . The seismic moment can then be defined as:

$$
M(t)=\frac{1}{3} \frac{\sigma_{S}}{\mu} 4 \pi \rho v_{p}^{2}\left(\frac{S_{0}}{\pi}\right)^{\frac{3}{2}} f(\tau),
$$

where $\sigma_{s}, \mu$, and $v_{p}$ are the strength, shear modulus, and $\mathrm{P}$ wave velocity of the impacted surface, respectively; $S_{o}$ is the surface area of the crater, and $f(\tau)$ is the normalized source function. Note that the mean of $f(\tau)$ is non-zero and that these forces are therefore compatible with a static deformation.

Figure 6 compares the relationships between crater size and momentum, and between seismic moment and crater size. For the relationship between crater size and momentum (Fig. 6a), different study cases are shown. The first set have been computed using the Holsapple and Housen web tool (http://keith.aa.washington.edu/craterdata/scaling/index.htm), 


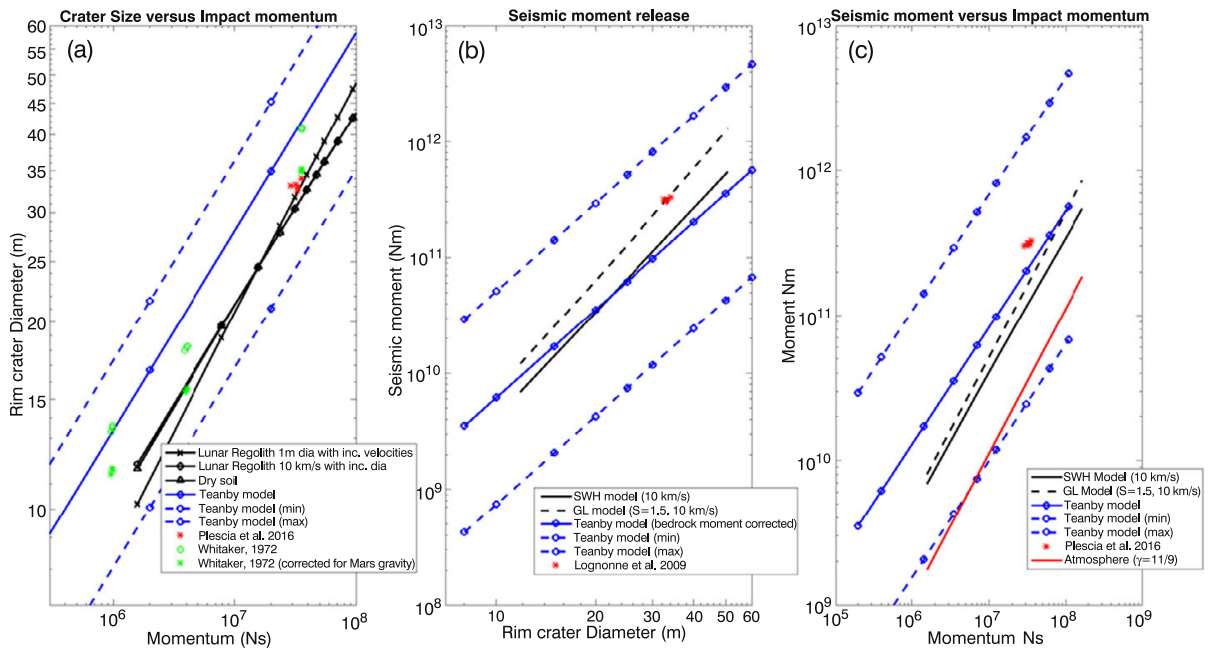

Fig. 6 (a) Diameter of resulting crater as a function of impactor momentum. The Holsapple web tool was used for cases shown in black (http://keith.aa.washington.edu/craterdata/scaling/index.htm). For the cases with constant mass, the velocities increase from $1 \mathrm{~km} / \mathrm{s}$ to $30 \mathrm{~km} / \mathrm{s}$, and a mass of $1571 \mathrm{~kg}$ is used, corresponding to a $1 \mathrm{~m}$ diameter impactor with density of $3000 \mathrm{~kg} / \mathrm{m}^{3}$. For the case with constant velocity, a velocity of $10 \mathrm{~km} / \mathrm{s}$ is assumed with increasing mass, all with the same density of $3000 \mathrm{~kg} / \mathrm{m}^{3}$. Different rheologies have been used for estimation of the crater size. The Teanby and Wookey (2011) relationship is shown in blue. Measurements of artificial lunar craters are shown in green (Whitaker 1972) and red (Plescia et al. 2016). (b) Comparison of the seismic moments from the SWH and GL models (black, solid and dashed lines, respectively). Note that the moments are very similar and could be adjusted easily with a small change of the $v_{p}$ velocity or the shear strength to modulus ratio. The apparent bedrock seismic moment using the Teanby and Wookey (2011) approach is also shown (blue). All moments are scaled for a bedrock velocity of $1 \mathrm{~km} / \mathrm{s}$ and a density of $2700 \mathrm{~kg} / \mathrm{m}^{3}$ by using the product of relation (10) and (11) of Sect. 3.4. (c) Relationship between seismic moment and impact momentum, showing a dependency close to linear. Note that for Apollo only the vertical component of the impact is used for momentum. All other examples are assumed to be perpendicular to the surface

for different types of impacted target material (lunar regolith, dry soil and soft rocks) and for impacts with either a constant impact velocity of $10 \mathrm{~km} / \mathrm{s}$ and increasing masses, or a constant mass and increasing velocities. Mars gravity $\left(g=3.71 \mathrm{~m} / \mathrm{s}^{2}\right)$ and $10 \mathrm{mbar}$ of pressure were assumed, as well as an impactor density of $3000 \mathrm{~kg} / \mathrm{m}^{3}$. These models are compared with the diameter of the crater of the Apollo SIVB and LM impacts, as measured by Whitaker (1972) and Plescia et al. (2016), as well as with the relationship proposed by Teanby and Wookey (2011). This suggests that the Teanby and Wookey (2011) relationship tends to over-estimate crater sizes with respect to the Holsapple model and lunar observations, although the diameters are within the error bars.

Figure $6 \mathrm{~b}$ provides the relation between the crater size and the seismic moment obtained by the GL and SWH models for different cases compared to those proposed by Teanby and Wookey (2011). For the GL model, which is shown for the case of $10 \mathrm{~km} / \mathrm{s}$ impacts in lunar regolith under Mars gravity, the ejecta amplification is set to $\left(1+0.3 \times v^{0.22}\right)$, with the impact velocity $v$ in $\mathrm{km} / \mathrm{s}$, following Lognonné et al. (2009). This provides an amplification factor of approximately 1.5. The GL model depends on the target material only through the amount of ejecta. The SWH model, on the other hand, depends only on the crater surface area and the ratio between shear strength and shear modulus, taken here to be 0.002. Seismic moments proposed by Lognonné et al. (2009) for Lunar Artificial SIVB impacts with the 

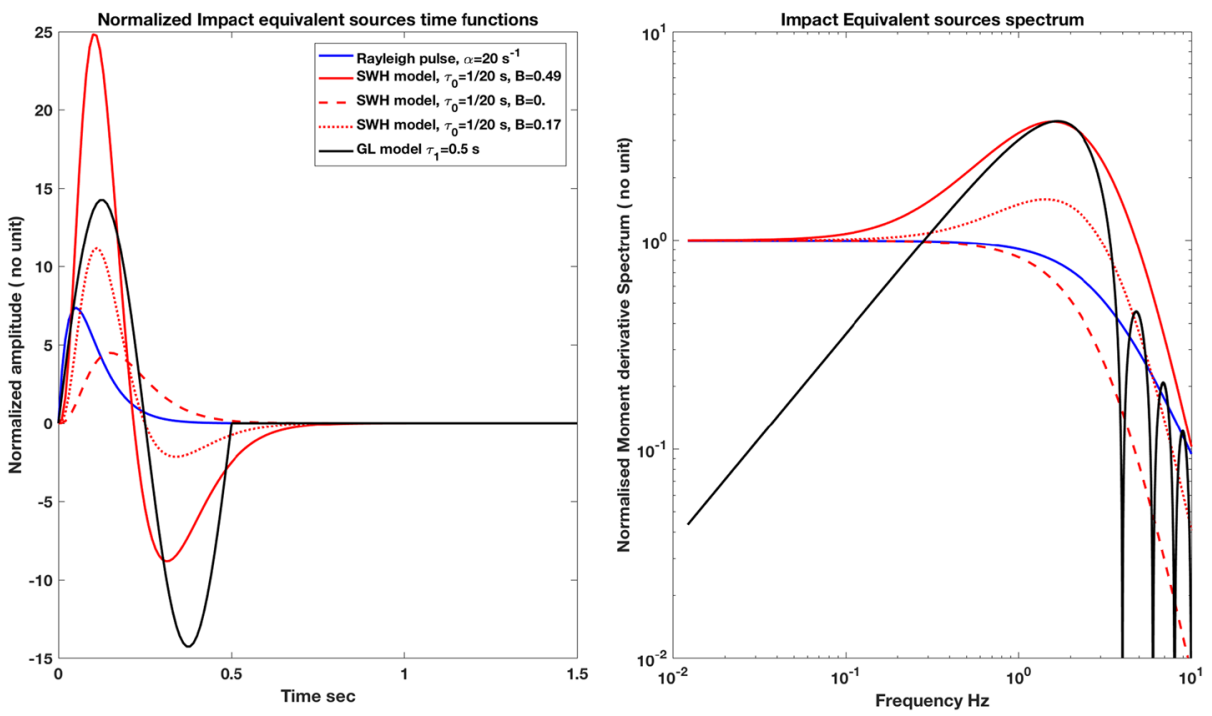

Fig. 7 (Left) Normalized source functions for a Rayleigh pulse $f_{R}(t)$ (blue), SWH model $\dot{g}(t)$ (red), and GL model $\dot{f}(t)$ (black). The SWH model is shown with three different values of the parameter $B: B=0$ (dashed red line), $B=0.171$ (dotted red line), and $B=0.49$ (solid red line). Values of $B=0.24$ and $B=0.49$ correspond to nuclear tests performed in granite and alluvium, with $\mathrm{P}$ velocities of 4.08 and $1.71 \mathrm{~km} / \mathrm{s}$, respectively. The solid black line is the GL source function. The parameters $\alpha$ (Rayleigh pulse) and $\tau_{0}$ are equal to $20 \mathrm{~s}^{-1}$ and $0.05 \mathrm{~s}$ respectively, while $\tau_{1}$ is taken as $0.5 \mathrm{~s}$ and has a cutoff frequency comparable to the $B=0.49 \mathrm{SWH}$ spectrum. (Right) Spectra of the same functions, which all have a similar cutoff frequency of $\sim 2 \mathrm{~Hz}$. Spectra for earthquakes with both $\omega^{2}$ and $\omega^{3}$ mechanisms will have a flat long-period spectrum comparable to those of the Rayleigh pulse or $B=0 \mathrm{SWH}$ models, without the overshoot of SWH when $B$ is not equal to zero

GL approach are shown, assuming for the latter the crater described in Plescia et al. (2016). Moments proposed by Teanby and Wookey (2011) are also shown but will be discussed later in the section related to seismic efficiency. As Teanby and Wookey used a moment to energy ratio based mostly on terrestrial shallow earthquakes, we assume $\mathrm{P}$ velocity and density of $5800 \mathrm{~m} / \mathrm{s}$ and $2700 \mathrm{~kg} / \mathrm{m}^{3}$ for their source region. For both the GL and SWH models, the regolith density and $\mathrm{P}$ velocity are set to $2000 \mathrm{~kg} / \mathrm{m}^{3}$ and $330 \mathrm{~m} / \mathrm{s}$ respectively. For the three models, we corrected the moment for a reference layer with P velocity of $1000 \mathrm{~m} / \mathrm{s}$ and density of $2700 \mathrm{~kg} / \mathrm{m}^{3}$, which is our reference model for Mars surface bedrock. We find a relatively good agreement between the different approaches within a factor of 2 in amplitude, which is \pm 0.2 in magnitude unit. All these approaches confirm that the seismic moment depends on the impactor momentum to the power $1 \pm 0.1$ (Fig. $6 \mathrm{c}$ ), and it is roughly proportional to the momentum, in accordance with the experimental observations presented in Sect. 3.4.

\subsection{Seismic Spectra, Cutoff Frequency, and Impact Momentum}

For the three models discussed in Sect. 3.1, Fig. 7 compares the normalized spectra of the seismic momentum derivative, as well as the displacement pulses. The case of the Rayleigh pulse of Sect. 3.1 is also shown. All curves represent the displacement seismogram or spectrum prior to its damping by seismic attenuation. Normalized source time functions and normalized spectra are shown for the GL model and the SWH model. The SWH model is 
Fig. 8 Cutoff frequencies for lunar impacts as a function of the reported impactor momentum (circles) as reported by Gudkova et al. (2015). The black line is the best fit scaling law found for $v_{p}=320 \mathrm{~m} / \mathrm{s}$ and a reference nuclear test performed in alluvium (Eq. (5))

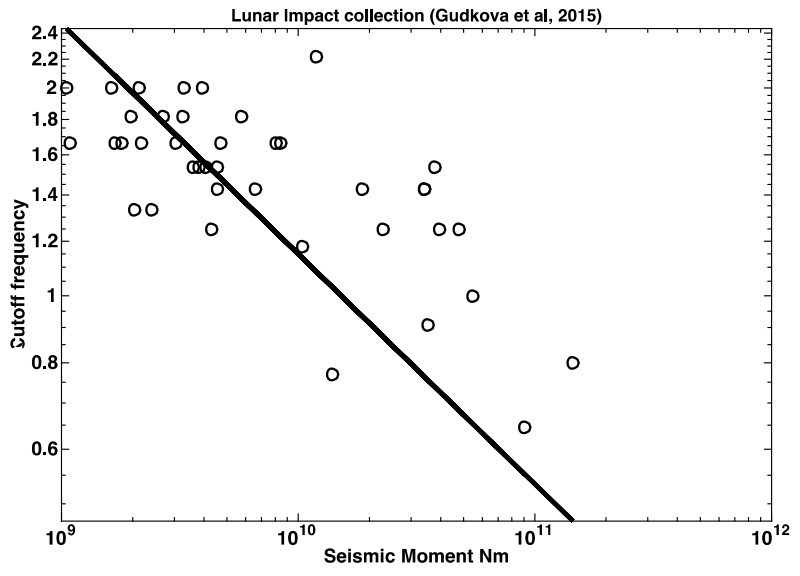

shown with two different values of $B$, the parameter in Eq. (3). Based on experiments with nuclear tests in various materials (Werth and Herbst 1963), measured values of $B$ are 0.05 in tuff, 0.17 in rock salt, 0.24 in granite, and 0.49 in alluvium (Shishkin 2007). Values of $B=0.0$ and 0.49 are shown in Fig. 7 as they encompass the other results.

The comparison with the GL model is interesting, as within the bandwidth of the Apollo data, $\sim 0.2 \mathrm{~Hz}-5 \mathrm{~Hz}$, the long period differences between the spectra were likely below the instrument resolution, and the shape of the spectra are therefore very similar. Note that the SWH models for large $B$ values have a frequency overshoot at body wave frequencies, which might increase the amplitudes of $1-2 \mathrm{~Hz}$ body waves by a factor of $\sim 4$. This is similar to the amplitudes observed in lunar data. Such overshoot also seems likely on Mars, as low-velocity materials are also expected in the subsurface (see Sect. 3.4).

The key difference between SWH and GL models is obviously the long period dependency of the spectrum. SWH spectra are flat at very long periods while the GL has a slope of $20 \mathrm{db}$ per decade. InSight data will be useful to determine which of the two models is a better match to observations. A key difference will be whether or not long-period surface waves are generated.

The cutoff frequency, which is proportional to the inverse of the time-duration of the seismic excitation process, is defined for the non-zero $B$ SWH or GL models as the peak of the displacement spectrum. While for $B=0$ or more classical tectonic quakes with $\omega^{2}$ and $\omega^{3}$ spectra, it is defined as the frequency for which the spectrum of displacement amplitudes has decayed by $\sqrt{2}$. This quantity will scale with the energy and propagation speed of the shock waves. From the scaling law of the elastic stored energy for the same process, we can expect this scaling to be:

$$
f_{\text {cutoff }}=f_{\text {ref }}\left(\frac{M}{M_{\text {ref }}}\right)^{-1 / 3}\left(\frac{v_{p}}{v_{\text {pref }}}\right)^{5 / 3},
$$

where $M, v_{p}$, and $f_{\text {cutoff }}$ are the seismic moment, P-wave velocity, and cutoff frequency, respectively; and $M_{r e f}, v_{\text {pref }}$, and $f_{\text {ref }}$ are those quantities for a reference event. We assume that the $v_{p} / v_{s}$ ratio and the $\sigma_{s} / \mu$ ratio are equivalent for both events. Figure 8 illustrates this scaling law for the lunar impact collection of Gudkova et al. (2015), for $v_{p}=300 \mathrm{~m} / \mathrm{s}$, $f_{\text {ref }}=1.15 \mathrm{~Hz}$ and $M_{\text {ref }}=10^{10} \mathrm{Nm}$. Most likely, the shallow subsurface seismic velocities on Mars will be larger than those of the Moon due to the less well-developed regolith. Larger velocities by a factor of $50 \%$ would shift the frequencies by a factor of two. Thus a 20 meter 
diameter crater associated with a $2 \times 10^{7} \mathrm{Ns}$ impulse and a $10^{10} \mathrm{Nm}$ seismic moment might have a cutoff frequency of $2.3 \mathrm{~Hz}$.

The cutoff frequency of an impact depends not only on the source size, but also on the properties of the impacted target material (e.g., porosity) (Lognonné et al. 2009; Gudkova et al. 2011). Modeling the variation in the cutoff frequency with the regolith porosity in the vicinity of the impact for the Moon shows that the larger the impact, the higher the impact duration, for impacts occurring in the same area of the surface. However, among impacts in different regions, this is not necessary valid. Differences between the source cutoff frequencies for impacts with the same momentum are caused by excitation processes in different geological regions and therefore by acceleration or deceleration of the shock wave associated with the collapse of subsurface porosity. The study by Gudkova et al. (2015) suggests a sensitivity of the cutoff frequency to the regolith porosity: the lower the timeduration of the process, the lower the maturity of the regolith. Similar analysis of future impact seismic data on Mars might enable remote investigation of the lateral variations in the Martian regolith.

\subsection{Seismic Efficiency}

The second approach developed to estimate the amplitude of seismic waves is based on the energy of the impact. A large portion of an impact's energy will be released as heat, and a small portion will be converted to seismic energy. The seismic efficiency, $k$, is defined as the ratio of the seismic energy produced by an impact $\left(E_{s}\right)$ to the kinetic energy of the bolide (or the yield of an explosion, $E$ ). This parameter describes the fraction of the kinetic energy of the object that is converted into seismic energy in the form of seismic waves (McGarr et al. 1969; Latham et al. 1970b; Patton and Walter 1993; Walker 2003; Teanby and Wookey 2011).

Empirical quantification of $k$ is very difficult as it requires integration of the entire seismic wave field, and the seismic efficiency differs widely between impacts, surface explosions and buried explosions. Due to the lack of high signal to noise impact events on Earth, $k$ has been estimated from numerical models (Walker 2003; Güldemeister and Wünnemann 2017) and scaling laws (Shishkin 2007), laboratory experiments (McGarr et al. 1969; Richardson and Kedar 2013 and Sect. 3.4), nuclear detonations (Pomeroy 1963; Patton and Walter 1993), missile impacts (Latham et al. 1970b), and artificial lunar impacts (Latham et al. 1970a). These events can differ from impacts in their physical processes, temporal and/or spatial scales, and their energies. The derived values span five orders of magnitude from $k=10^{-6}-10^{-1}$. Some of this broad range can be attributed to incomplete coverage of the seismic wavefield or frequency limitations of the recording seismic instruments. However, there is also likely to be a large scenario-dependent component that depends upon the surface material properties and properties of the impactor, such as density and speed.

Experimental values range from $10^{-5}$ to $10^{-3}$ for impacts on bonded sand (McGarr et al. 1969; Richardson and Kedar 2013). On the other hand, the artificial impacts of the Apollo 12 and 13 Saturn boosters, which had energy seven orders of magnitude larger, gave smaller values of $10^{-6}$ to $10^{-5}$ (Latham et al. 1970a). Underground explosions have much higher seismic efficiencies of $10^{-2}$ to $10^{-1}$ (Patton and Walter 1993). While explosive sources may approximate some of the processes found in impacts, these phenomena clearly differ in their physics. The seismic efficiencies obtained from chemical and nuclear explosions do not necessarily capture the momentum transfer dominated source mechanisms found in high velocity impacts. Generally, though, seismic efficiency is coupled to target properties: high seismic efficiencies $\left(k>10^{-3}\right)$ are typically found in explosions and nuclear tests in 
bedrock or highly consolidated materials (e.g., Patton and Walter 1993), while low seismic efficiencies $\left(k<10^{-5}\right)$ are seen in sediments or unbonded sands or soils (McGarr et al. 1969; Latham et al. 1970a). Recent studies for the Moon and Mars have used values of $10^{-6}$ (Davis 1993) and $2 \times 10^{-5}$ (Teanby and Wookey 2011). Lognonné et al. (2009) proposed that the seismic efficiency depends on both the seismic velocity at the point where the impact occurs and the duration of the source. They estimated $k=10^{-5}$ for a duration of $0.35 \mathrm{sec}$ in lunar regolith.

Shishkin (2007) suggests that the seismic efficiency for impacts is on the upper side for small impacts, with values of $10^{-3}$ or more for small impacts at Mach 10 with respect to the $\mathrm{P}$ wave seismic velocities. The GL model provides the ratio between seismic moment $\mathrm{M}$ and the kinetic energy as $k_{m}=2 S \frac{v_{p}}{v}$. It is therefore 2-3 times the inverse of the Mach ratio and will be about 1/10 for an impact at $10 \mathrm{~km} / \mathrm{s}$ over a surface with $350 \mathrm{~m} / \mathrm{s}$ P wave velocity. When combined with the ratio between seismic energy and moment:

$$
\frac{E_{s}}{M}=c \frac{\sigma}{\mu}
$$

with estimated values for $c$ of $0.22,0.27$, and 0.5 for impacts, explosions, and quakes, respectively, and a ratio $\frac{\sigma}{\mu}=2 \times 10^{-3}$, we get a seismic efficiency of $k=4-5 \times 10^{-5}$. This is comparable to experimental values (Latham et al. 1970b; McGarr et al. 1969). This ratio might be smaller on the Moon than on Mars, as impact velocities are larger and subsurface velocities are smaller, leading to a higher impactor Mach number.

\subsection{Experimental Determination of Seismic Source Time Function and Seismic Efficiency}

To experimentally measure some of these parameters, it is necessary to simulate the seismic signals expected from meteorite impacts on the Martian surface. Richardson and Kedar (2013) carried out a series of high velocity $(1-6 \mathrm{~km} / \mathrm{s})$ impact experiments at the NASA Ames Vertical Gun Range (AVGR) facility. The experiments spanned a variety of projectile impact velocities and angles and were carried out in near-vacuum to mimic Martian atmospheric conditions. Seismic sensors were embedded in target material analogous to the Martian surface, and they were digitally recorded at over 100,000 samples per second with seismic data loggers and high-speed cameras. A detailed experiment description will be summarized in a future paper. Here we summarize the key results and specific implications to the InSight mission.

In the experiment, 15 accelerometers were embedded in rows horizontally along the surface of a sand target, as well as below the impact point. These were used to measure signals from the impacting glass projectiles, which were used to derive both the seismic velocity $\left(V_{p}=250 \mathrm{~m} / \mathrm{s}\right)$ and quality factor $(Q \simeq 5)$ of the medium. We used the record from an accelerometer placed $0.2 \mathrm{~m}$ below the impact point to determine the source time function of the impact process. This was done by deconvolving the impulse response of the medium with the above properties from the seismic record. Once a source time function, $F(t)$ (force as a function of time), was determined, it was integrated and compared with the known momentum of the projectile, whose mass and speed were accurately measured for each shot. Table 2 compares the measured projectile momentum and the momentum estimated from the accelerometer records. In addition, seismic, efficiency was estimated from seismograms of three sensors at $0.2,0.4$, and $0.6 \mathrm{~m}$ below the impact point. 
Table 2 Experimental results for various projectile velocities. Comparison between projectile momentum measured in the lab and estimated from seismograms, and the resulting seismic efficiency estimates

\begin{tabular}{llll}
\hline $\begin{array}{l}\text { Projectile } \\
\text { velocity }(\mathrm{km} / \mathrm{s})\end{array}$ & $\begin{array}{l}\text { Measured projectile } \\
\text { momentum }(\mathrm{kg} \mathrm{m} / \mathrm{s})\end{array}$ & $\begin{array}{l}\text { Estimated projectile } \\
\text { momentum }(\mathrm{kg} \mathrm{m} / \mathrm{s})\end{array}$ & Seismic efficiency, $k$ \\
\hline 0.95 & 0.28 & 0.37 & $3.1 \times 10^{-3} \pm 0.7 \times 10^{-3}$ \\
2.23 & 0.66 & 0.68 & $1.3 \times 10^{-3} \pm 0.7 \times 10^{-3}$ \\
2.68 & 0.80 & 0.82 & $1.3 \times 10^{-3} \pm 0.7 \times 10^{-3}$ \\
4.68 & 1.39 & 1.43 & $1.4 \times 10^{-3} \pm 1.0 \times 10^{-3}$ \\
5.47 & 2.05 & 2.05 & $2.1 \times 10^{-3} \pm 2.0 \times 10^{-3}$ \\
\hline
\end{tabular}

The generally good agreement between the measured and estimated projectile momentum serves as an independent confirmation of the measured material properties $\left(V_{p}\right.$ and $\left.Q\right)$, and lends credence to the estimated source time function, $F(t)$.

Other impact experiments (e.g., Güldemeister and Wünnemann 2017) worked in the same impact speed range as in Table 2 but impacted quartz $\left(k=3 \times 10^{-3}\right)$, sandstone with $20 \%$ porosity $\left(k=2.56 \times 10^{-3}\right)$, and tuff with $43 \%$ porosity $\left(k=2.02 \times 10^{-3}\right)$. They used numerical impact hydrocodes to reproduce these impact events and calculate the seismic efficiencies.

The large uncertainty in impact seismic efficiency is due to the difficulty in accurately estimating $E_{s}$ from a seismogram. This requires assumptions about poorly known seismic energy flux, which depends on source geometry and material properties. However, once $F(t)$ is determined with a high degree of confidence, it can be used to estimate $E_{s}$. We do this, using a method routinely employed in the analysis of seismic waves emanating from an explosion source (Helmberger and Hadley 1981), in which a simple yet integrable mathematical function is used to represent $F(t)$.

We can represent $F(t)$ by a function known as a Jeffreys Pulse:

$$
f_{J}(t)=c t e^{-\alpha t}
$$

where $c$ is a constant of integration with units of force per unit time, and $\alpha$ is a characteristic decay time estimated from $F(t)$. By definition, the impact impulse is

$$
P \equiv \int_{0}^{\infty} F(t) d t=m v
$$

where $m$ is the mass of the projectile and $v$ is its velocity. Substituting $f_{J}(t)$ for $F(t)$, it can be shown that

$$
c=\alpha^{2} P
$$

Figure 9 shows a comparison between the estimated $F(t)$ and its representation as a Jeffreys pulse $f_{J}(t)$, showing the close match between our estimated source time function and that measured in the experiment.

The seismic efficiency values summarized in Table 2 are a few times larger than the incrater estimates $\left(5.7 \times 10^{-4}\right)$ obtained in laboratory experiments by Yasui et al. (2015). As pointed out by Yasui et al. (2015), however, estimates of the seismic efficiency from measurements outside the crater rim are substantially lower, which to some degree accounts for the wide range of seismic efficiencies quoted in the literature. As a result, the use of seismic efficiency in modeling of impacts introduces a substantial uncertainty. Using the source time 
Fig. 9 A comparison between the estimated source time function (red) for a vertical $1000 \mathrm{~m} / \mathrm{s}$ shot and its mathematical representation (blue) as a Jeffreys pulse

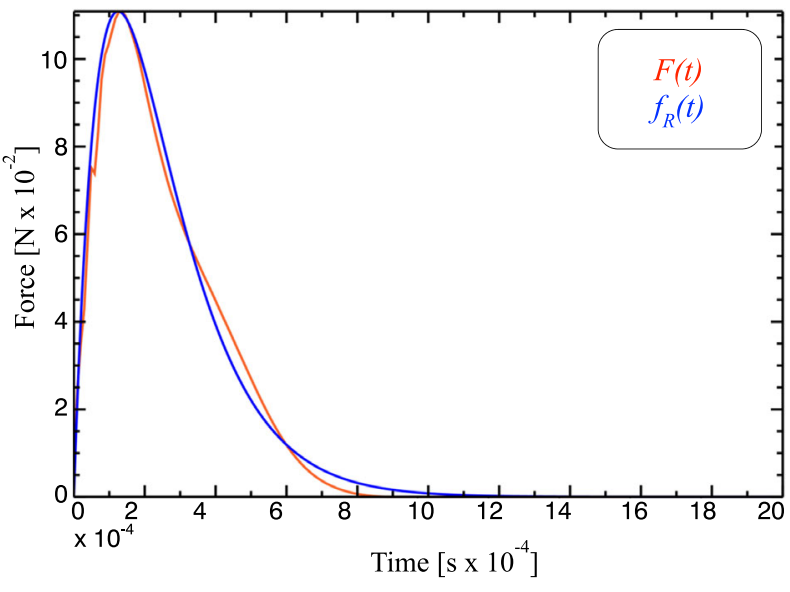

function enables a more accurate estimate of the impact force time history based on a known empirical crater-size - momentum relationship (Melosh 1989), and so eliminates the need to rely on the highly variable seismic efficiency factor. Using this strategy, we anticipate that newly discovered Martian impacts by InSight could be more accurately used for inverting for Martian interior properties.

When attempting to link seismic moment to the observed seismic efficiencies from tests, it is important to take into account that impact sources are not usually located in bedrock, but in brecciated material with low seismic velocities. For the same seismic moment, this leads to amplitudes larger by a factor of

$$
T_{m}=\frac{\rho_{b r} v_{p b r}^{3}}{\rho v_{p}^{3}},
$$

where $\rho_{b r}$ and $\rho$ are the densities of the bedrock and regolith, respectively, and $v_{p b r}$ and $v_{p}$ are the $\mathrm{P}$-wave seismic velocities in each. On the other hand, only a fraction of the amplitude of the wave will be transmitted to the underlying bedrock and thus could be detected remotely. The transmission coefficient for this is approximated as

$$
T_{m}=\frac{2 \rho v_{p}}{\rho_{b r} v_{p b r}+\rho v_{p}} .
$$

When compared to quakes occurring in bedrock, the moment above shall therefore be multiplied by the two conversion factors, Eqs. (10) and (11). The amplitude then depends only on the bedrock density and on the regolith and bedrock velocities. For a ratio of e.g. 10 between the surface velocity and that in the seismic crust, this will lead to magnitudes a factor of 1.5 larger. For example, a typical $10^{10} \mathrm{Nm}$ moment impact associated with a $20 \mathrm{~m}$ crater would only be a magnitude 0.65 event. This would be comparable to a quake of magnitude 2.15 in terms of seismic amplitudes, with a possible overshoot at $1-2 \mathrm{~Hz}$ of $4 \pm 1$ leading to body waves at $1 \mathrm{~Hz}$, close to those from a magnitude 2.5 seismic event. This type of effect is illustrated in Fig. 6b, where we compare the three models of seismic sources with the seismic moment provided by Teanby and Wookey (2011), all scaled for bedrock properties comparable to those of the Moon $\left(v_{p b r}=1000 \mathrm{~m} / \mathrm{s}\right.$ and $\left.\rho_{b r}=2700 \mathrm{~m} / \mathrm{s}\right)$. With these modifications, the two seismic source-based models, SWH and GL, and the seismic 
efficiency-based model (Teanby and Wookey 2011) then agree well with the Apollo recorded observations.

As the exact value of the seismic efficiency remains by its nature uncertain, we will use a fixed value of $5 \times 10^{-4}$ in InSight impact detection studies when needed. We judge this to be the current best estimate of $k$. It is within an order of magnitude of most other literature estimates and the AVGR impact experiments by Richardson and Kedar (2013) described in this section. As further evidence that this value is appropriate, it brings disparate methods into rough agreement: Teanby and Wookey (2011) use a modelling approach to impact detection, whereas Teanby (2015) uses an independent empirical based scaling relation. Agreement between the two methods is obtained if $k=5 \times 10^{-4}$ is used, suggesting this value is a good estimate. There is still likely to be an order of magnitude error in those results, though, due to scatter in the data used by Teanby (2015). Given the variations between values found by various authors, we still consider this value to have an order of magnitude uncertainty, because the efficiency is expected to depend on properties of the impact (momentum, velocity, impact angle, etc.) and the seismic properties of the impacted surface material.

\subsection{Shallow Subsurface Effects}

Much of the above theory was developed assuming a perfect medium in which the seismic waves travel from the source (impact site) to the detector (SEIS deployment location at the InSight landing site). However, the specific material properties of those two locations, as well as the path between them, will also affect the seismic signals received. This is true for impacts as well as for tectonic events, with the difference being that with impacts, we have a chance of identifying the precise source and then investigating the local geology at that location.

Understanding the material properties at the landing site are important for interpretation of any received signals. The presence of a surface layer of fragmented, loose regolith will both amplify and trap seismic waves; and the relatively high porosity of the regolith will affect the seismic efficiency. In comparison to earthquakes or marsquakes, these effects might be further amplified by the fact that the body waves from impacts will likely be relatively high frequency. When they are detectable, they will be in a frequency bandwidth of 0.5 to $5 \mathrm{~Hz}$ (Sect. 3.2), leading to possible site effects at high frequencies due to the expected low seismic velocities in the shallow subsurface (Delage et al. 2017).

\subsubsection{Material Effects at Detector Site}

In general, geophysical knowledge of a priori subsurface structure of Mars is based on a combination of orbital and in situ observations: HiRISE (High Resolution Imaging Science Experiment; McEwen et al. 2007), CTX (Context camera; Malin et al. 2007) and CRISM (Compact Reconnaissance Imaging Spectrometer for Mars; Murchie et al. 2007) images from the Mars Reconnaissance Orbiter (MRO), the radar and thermophysical properties of the surface materials, including albedo, thermal inertia and radar reflectivity (and inferred bulk density) (e.g., Golombek et al. 2008). Our knowledge of the material properties of the local InSight region come from remote sensing data studied extensively when selecting the InSight landing site (Golombek et al. 2017). The selected landing site is located in western Elysium Planitia at $4.5^{\circ} \mathrm{N}, 136.0^{\circ} \mathrm{E}$ at an elevation of $-2.6 \mathrm{~km}$. This is just north of the global dichotomy boundary between elevated heavily cratered southern highlands and lower standing, less cratered, northern plains. The landing site is on Hesperian basaltic lava plains that are $\sim 200 \mathrm{~m}$ thick and are underlain by sediments. Moderately low thermal inertia 
and measurement of rocks in high-resolution images show the regolith has few rocks and is composed of dominantly cohesionless sand or very weakly cemented soils (Golombek et al. 2017). Impact and eolian processes have created a fragmented regolith 3-17 m thick, which grades into coarse, blocky ejecta overlying strong, jointed bedrock (Warner et al. 2017). This bedrock is a $\sim 200 \mathrm{~m}$ thick stack of layered lava flows, possibly interbedded by ash and sedimentary deposits (Golombek et al. 2018, this issue). Knapmeyer-Endrun et al. (2017) used this stratigraphy, along with laboratory measurements (Delage et al. 2017), to develop a model of elastic properties with a rapid stepwise increase in seismic velocity and seismic attenuation Q with depth. See also Morgan et al. (2018, this issue) for a pre-landing assessment of regolith properties at the landing site. Data from the $\mathrm{HP}^{3}$ hammering (Kedar et al. 2017; Spohn et al. 2018, this issue) will tightly constrain local regolith properties and subsurface geology before science monitoring begins.

\subsubsection{Material Effects at Impact Site}

Influence on Seismic Amplitudes As noted in Sect. 3.1 and in Table 1, all source models generate seismic amplitudes that are proportional to the inverse of the seismic velocities where the source associated with the impact is released. This amplification effect due to the regolith is essential in the modeling of the amplitudes of the waves. In addition, the regolith will trap seismic waves (Fig. 10). This trapping will not only generate shallow layer surface waves, but also a ringing/reverberation effect of the direct body waves.

Influence on Seismic Efficiency The large variation in empirical estimates of seismic efficiency $k$ is likely to be partially attributable to differences in surface and subsurface material properties. However, the variability in scale, source, and material type makes it difficult to isolate the influence of specific material properties. A notable exception is the influence of porosity and water saturation on $k$, which were investigated numerically by Güldemeister and Wünnemann (2017). Compaction of dry and wet porosity close to the impact site absorbs energy from the shock wave, reducing the energy available to be radiated as seismic waves. Numerical simulations of 12-mm diameter iron impactors striking sandstone targets of various degrees of porosity and water saturation at $4.6 \mathrm{~km} / \mathrm{s}$ showed a factor of two reduction in seismic efficiency when porosity was increased from 0 to $40 \%$. An order of magnitude reduction in efficiency was seen when the pore space was filled with water. The rather modest reduction in $k$ with dry porosity may have been influenced by the model assumption that the shear strength of the sandstone targets was independent of porosity. A decrease in strength with increasing porosity would likely amplify the observed reduction in $k$, and may explain, in part, the low seismic efficiency inferred from impacts in the porous lunar regolith (Latham et al. 1970b). We expect the InSight region to be covered in fractured regolith, but not as porous as the upper layers of the Moon.

\subsection{Seismic Signals from Airbursts and Associated Seismic Source}

If an impactor's mass is comparable to or smaller than the mass of atmosphere it encounters, it will decelerate, ablate and potentially disrupt. This process rapidly transfers a large proportion, if not all, the impactor's kinetic energy to the atmosphere, producing a so-called airburst. Airbursts release the impactor energy into heat and therefore atmospheric overpressure with a much larger efficiency than the seismic efficiency discussed in Sect. 3.3. From Sedov shock wave theory (Landau and Liftshitz 1982) and for the Shoemaker-Levy 9 impact on Jupiter, Lognonné et al. (1994) estimated the seismic efficiency of an impact releasing its thermal energy in the atmosphere as larger than $(\boldsymbol{\gamma}-1)$, where $\boldsymbol{\gamma}$ is the adiabatic 


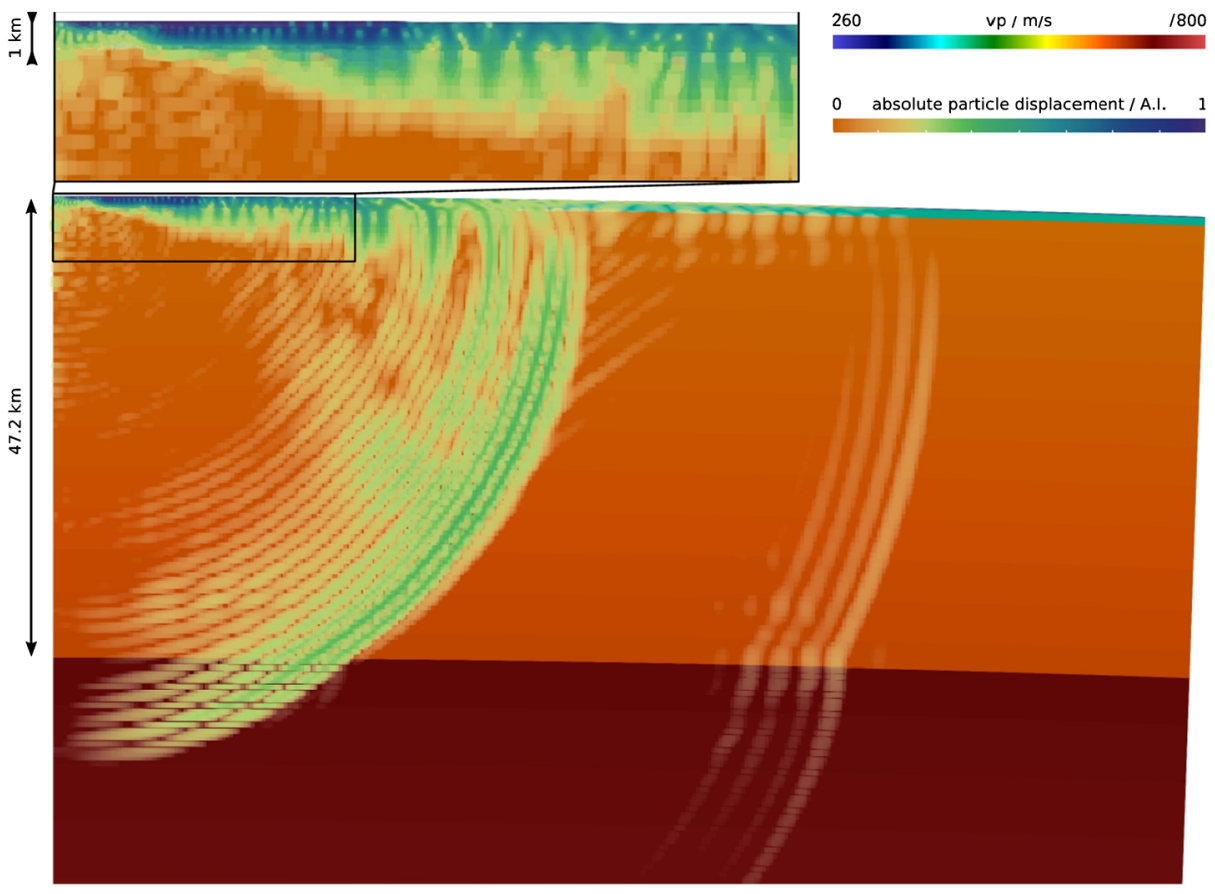

Fig. 10 Wavefield simulation for a short period of $1 \mathrm{~Hz}$ for a vertical impact in a $1 \mathrm{D}$ model with regolith $\left(80 \mathrm{~m}, v_{p}=265-600 \mathrm{~m} / \mathrm{s}\right)$, bedrock $\left(1 \mathrm{~km}, v_{p}=2700 \mathrm{~m} / \mathrm{s}\right)$ and a crustal layer $\left(47.2 \mathrm{~km}, v_{p}=5400-5730 \mathrm{~m} / \mathrm{s}\right)$. The color scale in the background indicates the p-wave velocity; the color scale in the foreground the absolute particle displacement. The shallow layers lead to complex waveforms in the body waves due to reverberation, and they trap energy due to total reflection acting as a wave guide. Furthermore, large amplitude short period surface waves with very low phase velocities are excited, though these can be considered an artifact due to the unrealistic homogeneity in the shallow layers

index. For high temperature $\mathrm{CO}_{2}$, this produces a seismic moment of more than 0.2 times the impactor energy, and therefore several orders of magnitude larger than the one associated with the ratio between seismic moment and energy. For $v_{p}$, this is equal to $2 v_{p} S v$ for the GL model described in Sect. 3.2, where $v_{p}, v$ and $S$ are the P wave's velocity, impactor velocity, and ejecta amplification, respectively. However, only a fraction of the airburst is converted to coupled seismic waves, with transmission coefficient $C=\frac{2 \rho c}{\rho c+\rho_{g} v_{p}}$ (see Sect. 3.3). For body waves, the ratio between the amplitude of the seismic waves excited by the impact on the surface and by the airburst near the surface can then be estimated as:

$$
\frac{\frac{(\gamma-1)}{2} m v^{2}}{4 \pi \rho c^{3}} T / \frac{S m v}{4 \pi \rho_{g} v_{p}^{2}}=\frac{(\gamma-1)}{2 S} \frac{v}{v_{p}} \frac{\rho_{g} v_{p}^{3}}{\rho c^{3}} T=\frac{(\gamma-1)}{S} \frac{v_{p} v}{c^{2}} .
$$

With $v_{p}$ 2-3 times larger than the sound speed and an impact velocity of Mach 40 $(\sim 14 \mathrm{~km} / \mathrm{s}$ ), this leads to a ratio larger than 10 . The amplitudes of seismic waves generated by the airburst as seismic sources are expected to be at least one order of magnitude larger than those of the surface impact itself, leading to $\sim 10 \times$ as many detections of these phases, as proposed in Sect. 4.5.

The same is valid for surface waves. This can be shown by comparing the excitation processes for seismic moment release either below or above the surface. Figure 11 compares 


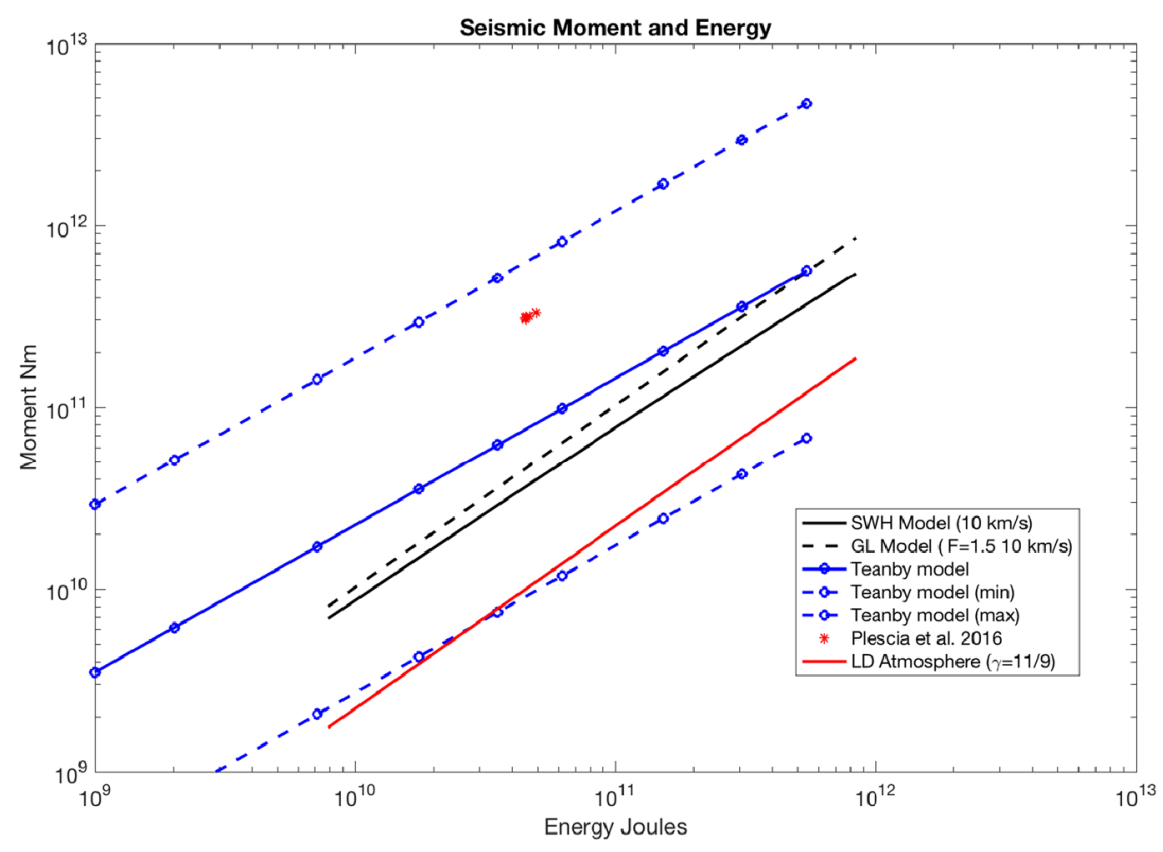

Fig. 11 Comparison of the relation between Seismic Moment and released energy for the Teanby (blue lines), Shishkin-Werth and Herbst (SWH) (black solid line), and Gudkova-Lognonné (GL) (black dashed line) models for release in the subsurface, and Lognonné-Dahlen (LD) model (red solid line) for release in the atmosphere. See text for details of models. For all models based on moment release, the atmospheric moment (red) is expected to be 2-4 smaller than the solid moment (black and blue). The bedrock correction is made with relations (10) and (11) of Sect. 3.4 with the same densities and velocities as for Fig. 6

these moments for the different approaches described in Sects. 3.1-3.2 and compares them to the moment, as estimated by Lognonné et al. (1994) for atmospheric release. This suggests the latter is smaller than those of the SWH and GL by a factor of 2 to 4 , respectively. On the other hand, the amplitudes of surface waves for a pressure glut source associated with an explosion will be proportional to $u_{\ell}\left(r_{0}\right) \operatorname{div}\left(\vec{u}_{\ell}\left(r_{s}\right)\right)$ where $r_{0}$ is the radius/altitude measured at the surface, $r_{s}$ the radius/altitude of the source, div the divergence operator, $\vec{u}_{\ell}$ the vector displacement field of surface wave mode of angular order $\ell$, and $u_{\ell}$ the vertical component. Figure 12 shows the amplitude of the fundamental Rayleigh mode ${ }_{0} \mathrm{~S}_{2000},{ }_{0} \mathrm{~S}_{3000},{ }_{0} \mathrm{~S}_{4000}$ and ${ }_{0} \mathrm{~S}_{5000}$, with periods of $4.08 \mathrm{sec}, 2.87 \mathrm{sec}, 2.28 \mathrm{sec}$, and $1.95 \mathrm{sec}$, respectively, as well as the excitation amplitude for a seismic moment located at a given altitude, either in the solid planet or atmosphere. The seismic model used is EH45TcoldCrust1 (Rivoldini et al. 2011) with a regolith layer and is described with more detail by Smrekar et al. (2018, this issue), while the acoustic model is the LD model described by Lognonné et al. (2016), together with the viscosity and molecular relaxation model described above. Computations of normal modes are made following Lognonné et al. (1994) and detailed in Lognonné et al. (2016) for Martian air-coupled Rayleigh waves and modes. Due to the almost free surface boundary condition, a large drop of the amplitude divergence is observed at the surface. For the same moment release, the near-surface atmospheric pressure glut associated with airbursts can be 25-100 (at $4 \mathrm{sec}$ ) to 10 (at $2 \mathrm{sec}$ ) times larger, depending on the frequency and altitude. This makes the excitation of surface waves by airbursts in some cases more effective than moment release in the subsurface. 

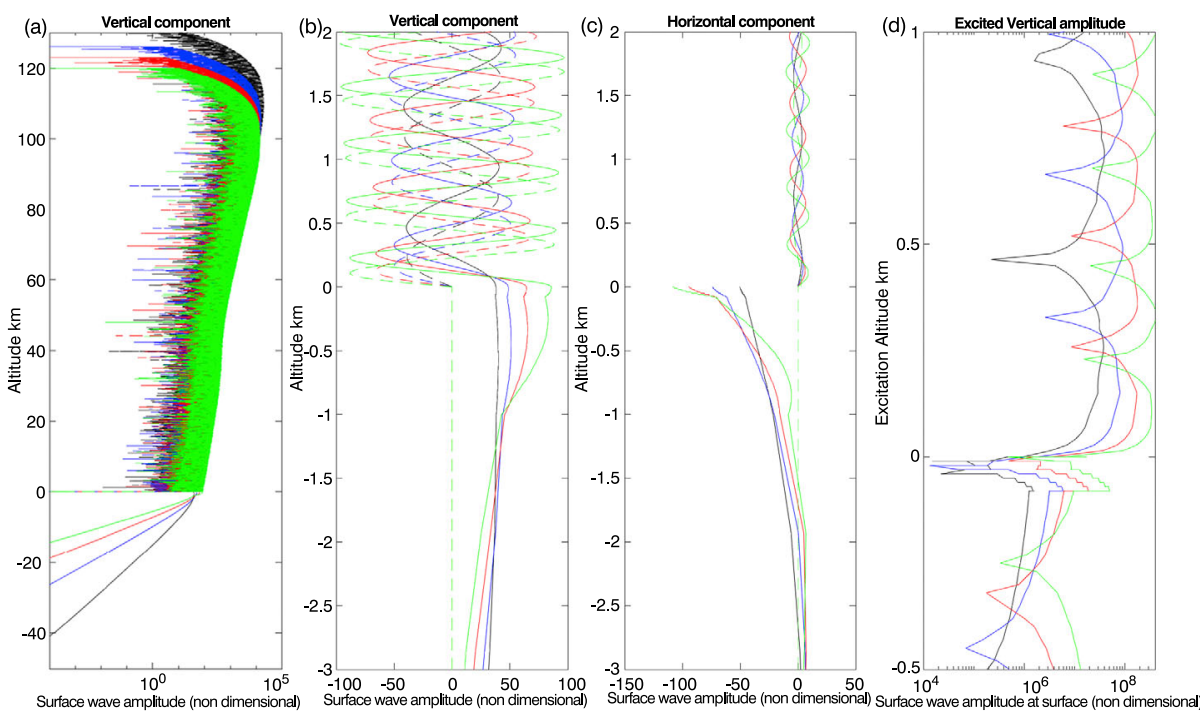

Fig. 12 Amplitude of the fundamental Rayleigh mode ${ }_{0} \mathrm{~S}_{2000}{ }_{0} \mathrm{~S}_{3000}{ }_{0} \mathrm{~S}_{4000}$ and ${ }_{0} \mathrm{~S}_{5000}$, with periods of $4.08 \mathrm{sec}, 2.87 \mathrm{sec}, 2.28 \mathrm{sec}$, and $1.95 \mathrm{sec}$, respectively, in black, blue, red and green. Solid lines are the real part, while dashed lines are the imaginary parts of the normal mode eigenfunctions. (a) shows the amplitude of the vertical component from a depth of $50 \mathrm{~km}$ (depths are negative on the y axis) to an altitude of $130 \mathrm{~km}$. Note the attenuation due to viscosity and molecular relaxation, occurring only at an altitude of $\sim 100 \mathrm{~km}$. (b) is the real and imaginary part of normal modes close to the surface. Note the quadrature structure of the real and imaginary components of the vertical component, showing the upward propagative aspects of the normal modes, as well as the continuity of displacement near the surface. (c) is the same as (b) for horizontal components. (d) is the amplitude of a mode at the surface of Mars, when the moment release is made at a given altitude. Note that at $4 \mathrm{sec}$, the amplitude for a release at $250 \mathrm{~m}$ altitude is larger by a factor of 25 than if the same moment is released at $80 \mathrm{~m}$ depth

\section{Impacts on Mars}

\subsection{Current Martian Impact Flux}

Predictions of the Martian impact rate are based on lunar crater densities, which have been tied to absolute ages with radiometric ages of returned samples (e.g. Hartmann 1966, 1977, 2005; Neukum and Wise 1976; Neukum and Ivanov 1994; Ivanov 2001). The calibrated lunar impact flux can then be extrapolated to Mars, taking into account estimates of the effects of the different impacting populations (size distribution and velocities of impactors), differing gravity that affects the final crater size for a given impactor, and atmospheric blocking at Mars. Until recently our understanding of the current Martian impact flux depended largely on the Mars/Moon cratering ratio, a value which was merely estimated based on these models.

Starting in the last decade with long-lived, high-resolution orbital imaging, new impacts have been detected appearing between successive images of the same area (Malin et al. 2006; Daubar et al. 2013). Using this technique, several hundred new, dated impacts have been discovered on Mars, several of which are very close to the InSight landing site (Daubar et al. 2015; Fig. 13).

The before- and after-imaging technique measures an impact rate of $1.65 \times$ $10^{-6}$ craters $/ \mathrm{km}^{2} / \mathrm{yr}$ with an effective diameter $\geq 3.9 \mathrm{~m}$ (Daubar et al. 2013). Below this 


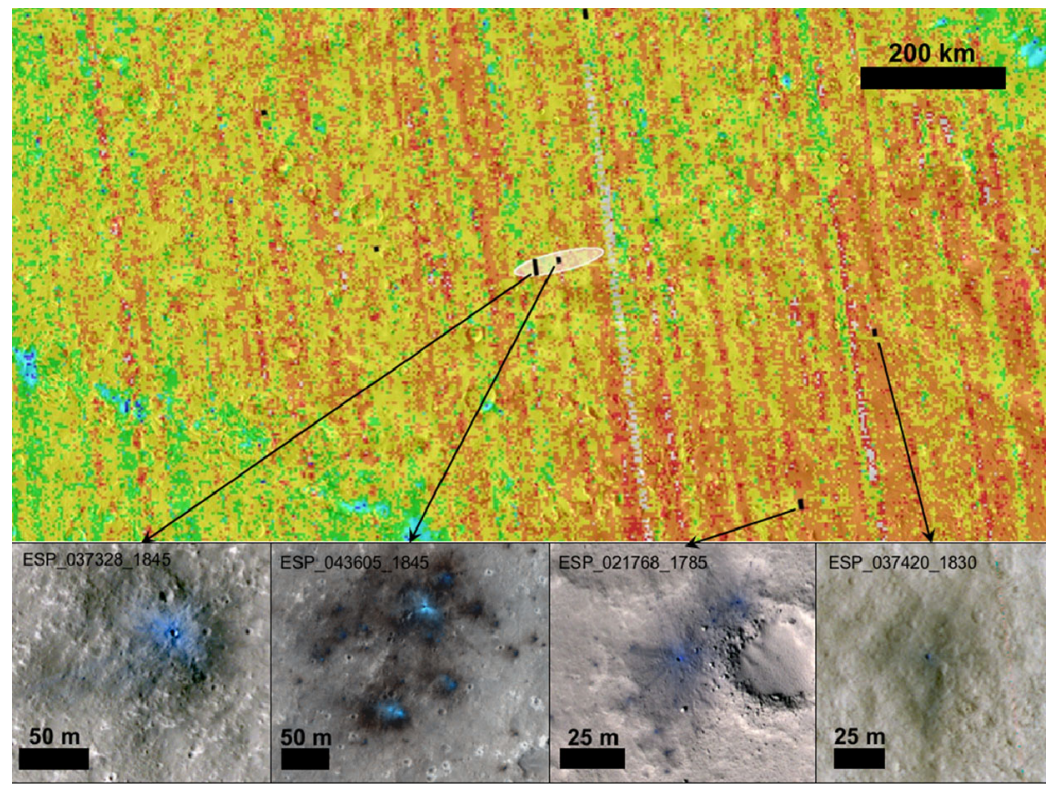

Fig. 13 Several new, dated impact craters discovered close to the InSight landing site. The final reference landing ellipse is shown in white $\left(4.5^{\circ} \mathrm{N}, 135.9^{\circ} \mathrm{E}\right)($ Golombek et al. 2017). HiRISE footprints containing new impact sites dated by before and after images are shown in black. Basemap is the THEMIS Day IR $100 \mathrm{~m}$ global mosaic v.11.5 (Edwards et al. 2011) overlain with the TES Dust Cover Index (Ruff and Christensen 2002), where red is high dust cover and blue is low; lower dust cover to the southwest is likely contributing to fewer craters being found there. HiRISE cutouts are from enhanced false color RDR products with North up; HiRISE images credit NASA/JPL/University of Arizona

size, a drop-off in the impact rate is seen, which could be due to resolution effects, atmospheric filtering, observational biases, or other factors.

In general, this technique allows for a direct measurement of the current impact rate at Mars. However, that measurement is biased by the limitations of imaging, such as spatial resolution and coverage. For these new impacts, there is also a detection bias that allows for discovery of new impacts only when there is a strong albedo contrast in an impact blast zone many times larger than the crater itself (Daubar et al. 2013). Fading of those low-albedo blast zones may also contribute to lack of small crater detections (Daubar et al. 2016). A seismic measurement of the current impact rate would be free of such biases, although there will be different biases in such a measurement, as discussed in Sect. 8.3. Lognonné et al. (2009) made such a measurement for current lunar impacts, and the seismically determined impact flux on the Moon was found to be within $\pm 50 \%$ of that at the top of the Earth's atmosphere.

\subsection{Martian Impactor Characteristics}

The impactors responsible for forming these new dated craters are presumably represented by the population of Mars-crossing objects (MCOs). This group of objects was studied in the past (JeongAhn and Malhotra 2015 and references therein) by selecting a subset of known asteroids from the Minor Planet Center orbital catalog. ${ }^{1}$ This set of MCOs was chosen to be

\footnotetext{
${ }^{1}$ http://www.minorplanetcenter.org/iau/MPCORB/MPCORB.DAT, download performed on Oct. 30th 2017.
} 

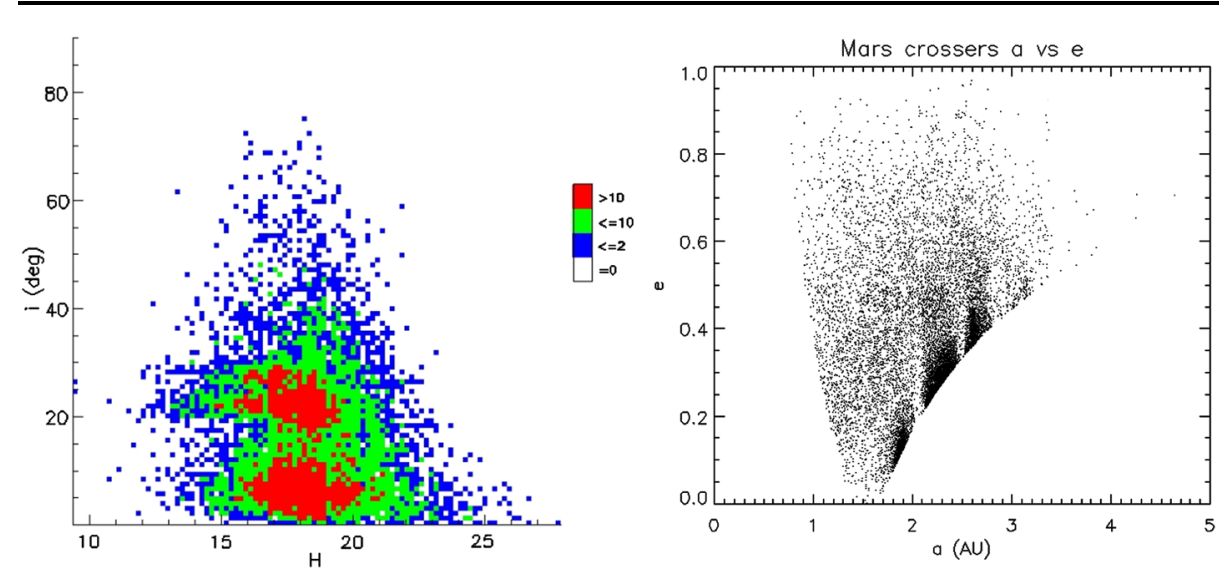

Fig. 14 Left: Distribution of inclination $(i)$ vs. absolute magnitude $(H)$ of the population of Mars-crossing object (MCOs), selecting based on perihelion and aphelion. Colors represent the number of objects with those values. Right: Semi-major axis $(a)$ vs. eccentricity $(e)$ of the same population. Gaps caused by resonances with Jupiter near $a=2.06,2.5$, and $3.27 \mathrm{AU}$ can be recognized

those with $Q>q_{\text {Mars }}$ and $q<Q_{\text {Mars }}$ (where $q$ is the perihelion distance, and $Q$ is the aphelion distance). Additionally, the selection was limited to objects that have been observed for more than one opposition. This leads to a population of 13355 MCOs, whose orbital distribution is shown in Fig. 14. Note that if we include the MCOs that have been observed during one opposition only, the total number of MCOs increases to 31207. That population has similar general trends as the downselected population. The two populations highlighted by previous studies, below and above $i=18^{\circ}$, are clearly visible in Fig. 14 . The absolute magnitude distribution shows that most of known MCOs are in the range 12-24 mag. Additional fainter MCOs are expected from future surveys, such as LSST (Large Synoptic Survey Telescope; LSST 2018).

The impact velocities and directions of MCOs are computed using the Neslusan et al. (1998) method, the source code for which was kindly provided by the authors. We modified the code to apply it to Mars. The advantage of this method is that it not only computes relative velocities, but also the location of the radiant (position of the sky where the impacting MCOs seem to come from), as well as the Solar Longitude $\left(L_{S}\right.$; ecliptic Longitude $+180^{\circ}$; a measure of Martian season) of the planet at the time of the closest approach. The distribution of the radiants and $L_{S}$ are showed in Figs. 15, 16, 17, and 18.

\subsection{Martian Crater Morphology}

Impact craters formed during the lifetime of the InSight mission are expected to be small $(<100 \mathrm{~m})$ simple craters. These will be similar to primary craters formed on Mars during recent monitoring by spacecraft (Daubar et al. 2013, 2014). These simple craters are bowl-shaped depressions, with a breccia lens accumulated at the bottom of the crater, and a depth-diameter ratio of $\sim$ 1:5 (Melosh 1989; Daubar et al. 2014). New small Martian craters seldom have an appreciable raised rim (Daubar et al. 2014), perhaps due to impacting a more porous upper layer of the Martian crust. In most cases, any morphological complexity in craters of this scale originate from inhomogeneities in the target, such as variable strength, density or porosity (e.g., Quaide and Oberbeck 1968; Senft and Stewart 2007). Features resulting from these inhomogeneities include irregular 
Fig. 15 Distribution of radiants of MCOs at Mars. RA: right ascension, DEC: declination (J2000). The colors represent the number of bodies with those parameters. A concentration of radiants can be recognized near $R A \sim 280^{\circ}, D E C \sim 30^{\circ}$

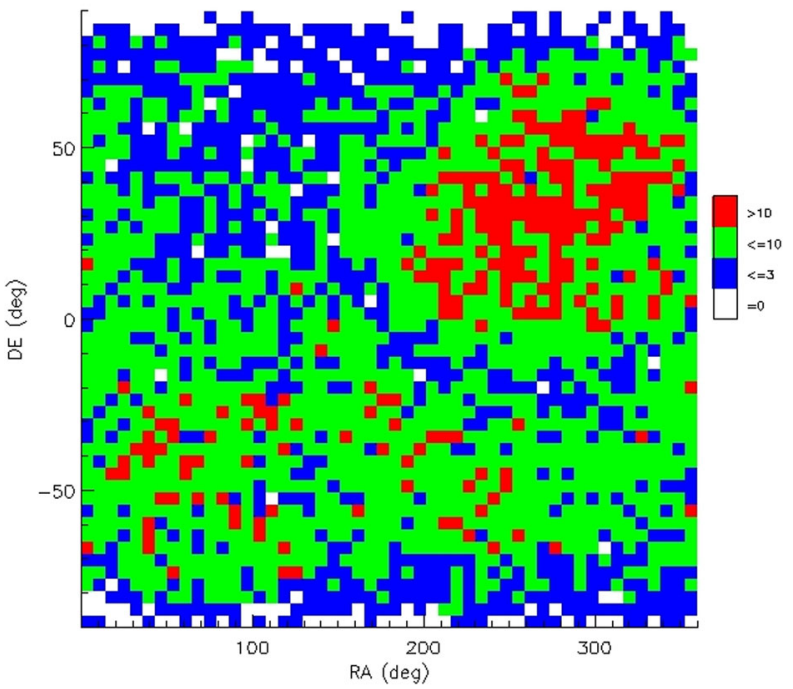

Fig. 16 The vector directions of relative velocities of MCOs as seen from Mars. The point at $[0 ; 0]$ is the Mars apex. The Sun is at $[-90 ; 0]$ and anti-Sun at [+90; 0]. Bands can be recognized, as a consequence of low and high inclination populations

Fig. 17 Histogram of Mars Solar Longitude $\left(L_{S}\right)$ at the time of closest encounter with each MCO. The maximum around $L_{S}=200^{\circ}$ is more pronounced if the criterion selection on the number of observed oppositions is relaxed
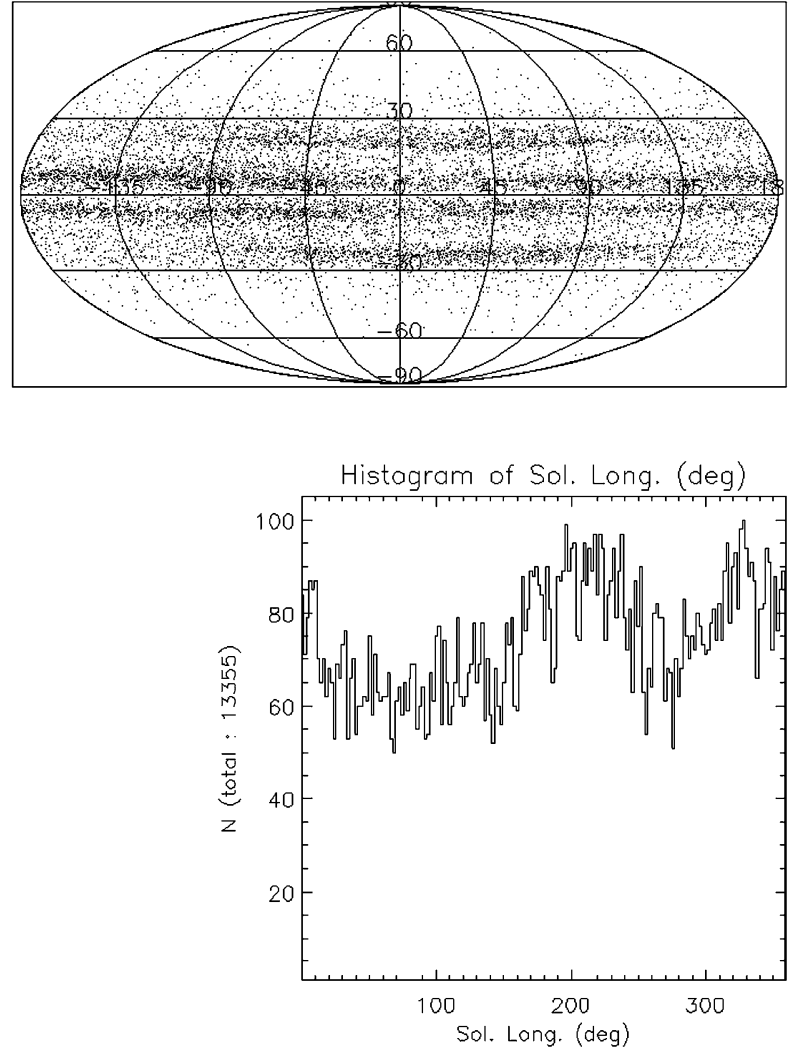

rims, flat floors, and "benches" or concentric craters (Daubar et al. 2014). At the InSight landing site, fresh rocky ejecta craters and nested craters indicate a fragmented regolith $3-$ 
Fig. 18 Distribution of the velocity of MCOs at the top of the Martian atmosphere. The median is located at $10.9 \mathrm{~km} / \mathrm{s}$ and the mean at $11.7 \mathrm{~km} / \mathrm{s}$. Two peaks can be seen at $\sim 6 \mathrm{~km} / \mathrm{s}$ and $\sim 11 \mathrm{~km} / \mathrm{s}$

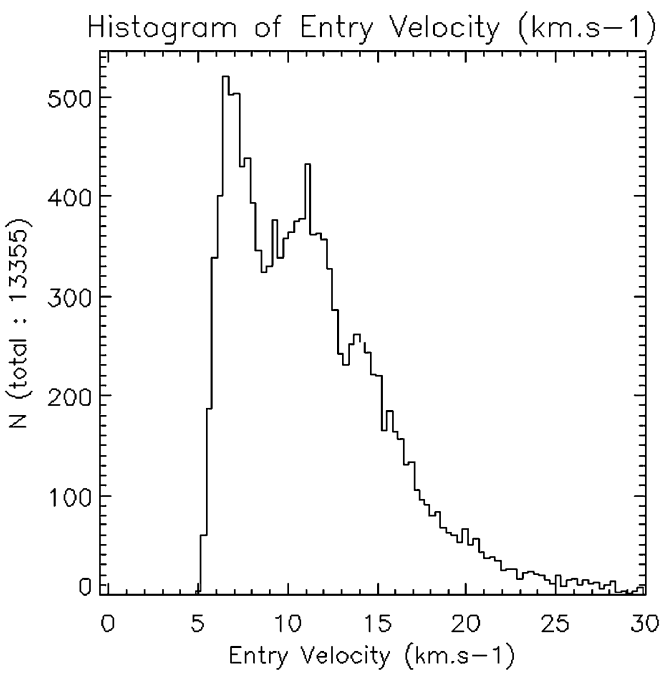

$17 \mathrm{~m}$ thick (Warner et al. 2017) and initial depth/diameter ratios about half that expected (Sweeney et al. 2016; Golombek et al. 2017), similar to other poorly consolidated targets on Mars (Watters et al. 2015).

Approximately half of such impacts form a single simple crater, while the other half form crater clusters, owing to the meteoroid fragmenting in the atmosphere before reaching the ground (Daubar et al. 2013). Given the prevalence of crater clusters, it is possible that a significant fraction of single craters also form by impact of a fragmented body, where fragments did not separate sufficiently to form separated craters (e.g., Miljković et al. 2013). The crater produced by such an impact would exhibit a shallower depth than if formed by a single consolidated impactor (Artemieva and Pierazzo 2009). This could account for some of the variation in depths of newly formed craters on Mars (Daubar et al. 2014), if shallower craters were created by this process.

\subsection{Martian Crater Scaling}

To connect the impactor energy and the crater diameter produced, Teanby and Wookey (2011) proposed a simple scaling equation relating crater size to the kinetic energy of the impactor, based on large-scale impact and explosion experiments. This formulation has the advantage of directly linking the observed crater size to seismic energy through the seismic efficiency. However, laboratory impact experiments and numerical simulations have shown that crater diameter does not scale simply with impact energy (e.g., Schmidt and Housen 1987; Holsapple 1993; Wunnemann et al. 2011). The most widely-used and successful crater scaling approach, commonly known as pi-group scaling, instead relates crater size to a combination of impactor energy and momentum, known as the coupling parameter (Holsapple and Schmidt 1987). The implication is that two impacts with the same kinetic energy but different combinations of impactor mass and velocity produce craters of different size. Moreover, the form of the scaling equation depends on the gravity, density, and cohesive strength of the target surface. In a cohesionless material, such as a dry granular regolith with negligible cohesion, crater size is limited by gravity; that is, the weight of the displaced material. In a cohesive soil or rock, on the other hand, crater size is limited by both gravity 


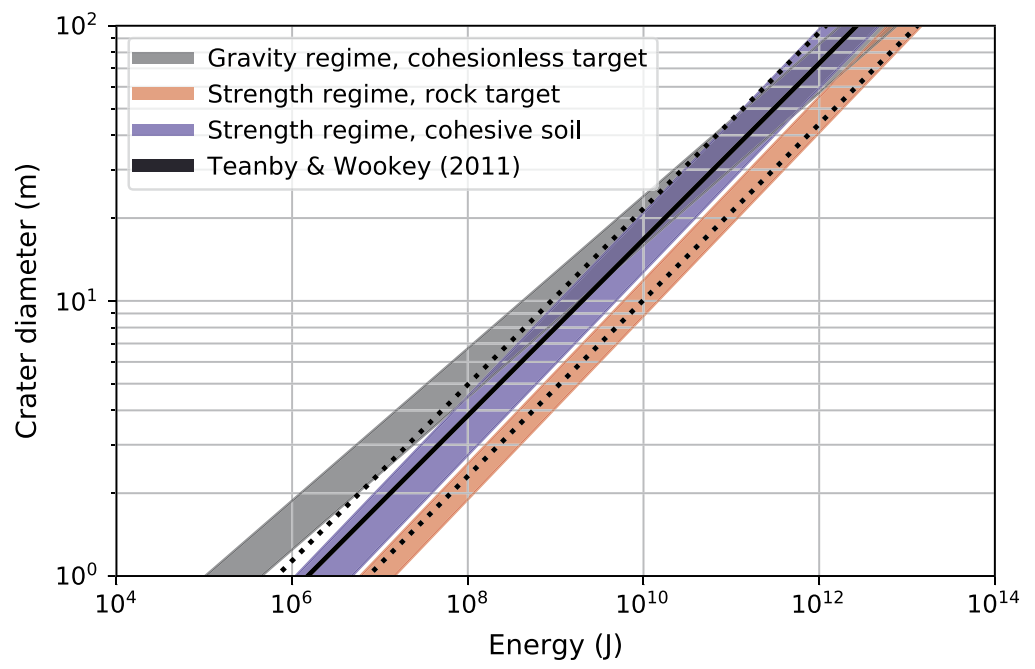

Fig. 19 Comparison of crater size scaling relationships for impact craters on Mars shown as a function of kinetic energy of the impactor. Pi-group crater scaling equations are shown as bands bounded above by a slow, dense impactor scenario and below by a fast, low-density impactor scenario. Bands are shown for three target surface approximations: a cohesionless regolith-like target with a density of $1.5 \mathrm{~g} / \mathrm{cc}$ and Martian gravity (grey); a cohesive soil/regolith of the same density, but with a small cohesive strength of $100 \mathrm{kPa}$ (blue); and a dense $(3 \mathrm{~g} / \mathrm{cc})$ rocky surface with a cohesive strength of $10 \mathrm{MPa}$ (red). Gravity is neglected in the latter two scenarios. Black lines show the impact energy-crater size scaling equation (dotted lines show minimum and maximum bounds) derived by Teanby and Wookey (2011)

and the strength of the material. In small craters, strength is dominant and the effect of gravity can be ignored, but in larger impacts gravity begins to dominate and strength effects can be neglected.

Figure 19 compares the impact energy-crater diameter scaling equation proposed by Teanby and Wookey (2011) with pi-group crater scaling equations (Schmidt and Housen 1987; Holsapple and Housen 2007) for the range of crater size most likely to be observed during the InSight mission. Pi-group scaling results are shown for three target approximations: a cohesionless regolith-like target with a density of $1.5 \mathrm{~g} / \mathrm{cc}$ and Martian gravity; a cohesive soil/regolith of the same density, but with a small cohesive strength of $100 \mathrm{kPa}$; and a dense $(3 \mathrm{~g} / \mathrm{cc})$ rocky surface with a cohesive strength of $10 \mathrm{MPa}$. Gravity is neglected in the latter two scenarios. In kinetic energy-crater diameter space, the pi-group scaling equations for each target approximation plot as a line only for a specific combination of impactor density and velocity. We therefore show a band of possible outcomes, bounded above by a slow, dense impactor scenario defined as an iron impactor $(7.9 \mathrm{~g} / \mathrm{cc})$ striking at Mars' escape velocity $(5 \mathrm{~km} / \mathrm{s})$. This is bounded below by a fast, low-density impactor scenario defined as an icy impactor $(1 \mathrm{~g} / \mathrm{cc})$ striking at $20 \mathrm{~km} / \mathrm{s}$. The analysis assumes vertical impact, neglects any deceleration during atmospheric entry, and accounts for a $30 \%$ difference between the crater diameter at the preimpact level and the final rim diameter (Holsapple 1993).

The comparison of scaling approximations illustrates that there is nearly a two order of magnitude range in impact energy required to produce a given crater size depending on the properties of the Martian surface and the density and speed of the impactor. The range of uncertainty reduces for larger craters or impacts known to be formed in regolith. Despite its simplicity, the energy scaling equation derived by Teanby and Wookey (2011) lies near 
the middle of the range of more conventional scaling approximations and the uncertainty attached to it is a good approximation of the variability in crater size scaling from anticipated variations in impactor and target properties. We also note that the pi-group scaling equations for cohesionless and cohesive soil/regolith intersect at a crater size of approximately $50 \mathrm{~m}$, implying that the cohesive strength of the upper tens of meters of the Martian surface will have an important control on the size of craters likely to be formed during the InSight mission $(<50 \mathrm{~m})$.

\subsection{Fragmentation in the Martian Atmosphere}

Unlike the Moon, Mars has enough of an atmosphere for it to be a factor when considering impacts and their seismic effects. When cometary or asteroidal material encounters a planetary atmosphere, aerodynamic resistance causes deceleration of the impacting body (meteoroid). If aerodynamic stresses are high enough, the meteoroid may experience ablation and/or fragmentation. Ablation occurs when sufficient heat is generated to vaporize or melt material from the surface of the meteoroid. In the thin Martian atmosphere ablation is near insignificant for all but very small meteoroids (sub-cm scale) entering at high speeds. Fragmentation is often assumed to occur when the stagnation pressure, $P=\rho_{a} v_{m}^{2}$, in front of the meteoroid is approximately equivalent to the meteoroid's bulk strength. Thus fragmentation is sensitive to entry velocity. After fragmentation, the effective surface area of the meteoroid increases as the fragments separate, dramatically increasing the rate of deceleration and energy loss to the atmosphere. Depending on the nature of fragmentation and rate of separation, such events can result in an airburst (a catastrophic disruption in the atmosphere) and/or near-simultaneous surface impact of a swarm of fragments to form a cluster or strewn field of craters. If fragmentation does not occur or occurs at very low altitude, the meteoroid will strike the ground as a basically coherent mass and form a single crater (e.g., Collins et al. 2005; Miljkovic et al. 2016).

In the absence of ablation and fragmentation, the deceleration of a single intact meteoroid is principally controlled by characteristics of the meteoroid (i.e. mass, shape, density) and its trajectory (i.e. velocity, angle of entry, atmospheric densities), and is well described by a simple drag equation (e.g. Baldwin and Sheaffer 1971). However, the fate of the meteoroid after fragmentation is much more complex to analyze and depends on highly-variable meteoroid strength (Popova et al. 2011), style of fragmentation (catastrophic vs. progressive), and the interaction between fragments and wake behavior (Passey and Melosh 1980; Ivanov et al. 1997; Chyba et al. 1993; Hills and Goda 1993; Register et al. 2017; Wheeler et al. 2017).

Of particular relevance to InSight is the fate of meter-scale meteoroids as seismic sources (Teanby and Wookey 2011; Stevanović et al. 2017). Forming decameter-scale craters, these are able to deliver the energy necessary for seismic detection (tens to hundreds of tons of TNT equivalent energy; 1 kton TNT $=4.185 \times 10^{12}$ Joules), whilst also being frequent enough that several to tens of events are expected throughout the mission (Teanby and Wookey 2011; Teanby 2015; Stevanović et al. 2017; Sect. 6.2). Observations of recently formed craters on Mars reveals that approximately half of current impacts of this scale result in single craters, while the other half undergo fragmentation in the atmosphere and form crater clusters (Daubar et al. 2013, 2018). This proportion of fragmentation events suggests a median effective strength of approximately $1 \mathrm{MPa}$ for meter-scale objects entering Mars' atmosphere, which is consistent with estimates of bulk meteoroid strength from terrestrial fireball observations (Popova et al. 2003, 2011), although a significant fraction of them seem to be weaker than this (Hartmann et al. 2017). For an approximately 1-m diameter ordinary 
chondrite meteoroid, a bulk strength of $1 \mathrm{MPa}$ would imply a fragmentation threshold entry speed of $8 \mathrm{~km} / \mathrm{s}$, assuming a trajectory $45^{\circ}$ from vertical at atmospheric entry. Meteoroids entering Mars's atmosphere between this speed and Mars's escape speed $(5 \mathrm{~km} / \mathrm{s})$ would tend to remain intact, losing less than $5 \%$ of their initial speed prior to forming a single crater. Meteoroids entering at higher speeds on the same trajectory would fragment at altitudes up to $30 \mathrm{~km}$ for an entry speed of $30 \mathrm{~km} / \mathrm{s}$. The most likely entry speeds for Mars are evenly distributed around peaks at $6.5 \mathrm{~km} / \mathrm{s}$ and $11.5 \mathrm{~km} / \mathrm{s}$ (Le Feuvre and Wieczorek 2011; Fig. 18). A $\sim 8 \mathrm{~km} / \mathrm{s}$ breakup threshold, between these two peaks, is therefore roughly consistent with the near-equal numbers of single and clustered impacts observed by Daubar et al. (2013). However, the mass, momentum and kinetic energy of the fragments before they strike the ground is highly dependent on the assumed model of fragmentation. If fragmentation is catastrophic, no sizeable fragment may strike the ground, but the resulting airburst may still be able to deliver seismic and acoustic signals to the SEIS detector depending on its altitude and the rate of energy deposition in the atmosphere (Stevanović et al. 2017). Hence, three classes of impact-related seismic sources might be recorded by the SEIS instrument: (i) surface impact of a single mass (no fragmentation); (ii) near-simultaneous surface impact of a swarm of meteoroid fragments, separated by a few tens to hundreds of meters; and (iii) airburst caused by catastrophic disruption and rapid energy deposition in the atmosphere. The first of these, single impacts, is the canonical case discussed primarily in Sect. 3 on impacts in general, and is nominally assumed in the rest of the paper. In the next sections we discuss the physical processes and expected seismic signals from clustered impacts and airbursts.

\subsubsection{Impact Clusters on Mars}

The seismic source for a cluster would behave differently than a singular impact; the energy of the impacts will be distributed over a larger area, typically between 10-1000 meters (Daubar et al. 2018). The source will be partitioned amongst craters of different sizes, and presumably bolides of various sizes. Schmerr et al. (2016) have built a seismological model for the predicted seismic signatures that would be recorded by seismometers deployed on Mars (Fig. 20). These source predictions are created using the measured crater properties from Daubar et al. (2018), along with a crater diameter scaling law for the strength regime (Holsapple and Housen 2007) and momentum-driven source model after Gudkova et al. $(2011,2015)$ to relate the expected magnitude of the seismic source to the observed crater properties (see also Sects. 3.1 and 4.4). The magnitude prediction is then combined with 3-D wave propagation modeling, using the Serpentine Wave Propagation Package (Petersson et al. 2010). The resulting theoretical Martian models are used to investigate the effect of a distributed source on the expected amplitudes of body and surface waves that will be essential for studying Martian internal structure.

The resultant source time function was found to be dependent upon the total moment release of the multiple impacts, relative timing of impact events, and geographic closeness (dispersion) of the clustered impacts. It was found that clusters have smaller peak amplitudes and more short-period energy in their source spectra compared to single crater impacts. While more numerous smaller craters in clusters contribute insignificant energy to the source function, they add to the complexity of recorded seismic energies and produce a more diffuse seismic signal (Fig. 20). With such diffuse signals, it will be more difficult to identify $\mathrm{P}$ wave arrivals and thus will add uncertainty to the identification of source location. However, being able to differentiate between seismic signals from single crater impacts and the more diffuse and complex signals from crater clusters will allow us to predetermine 

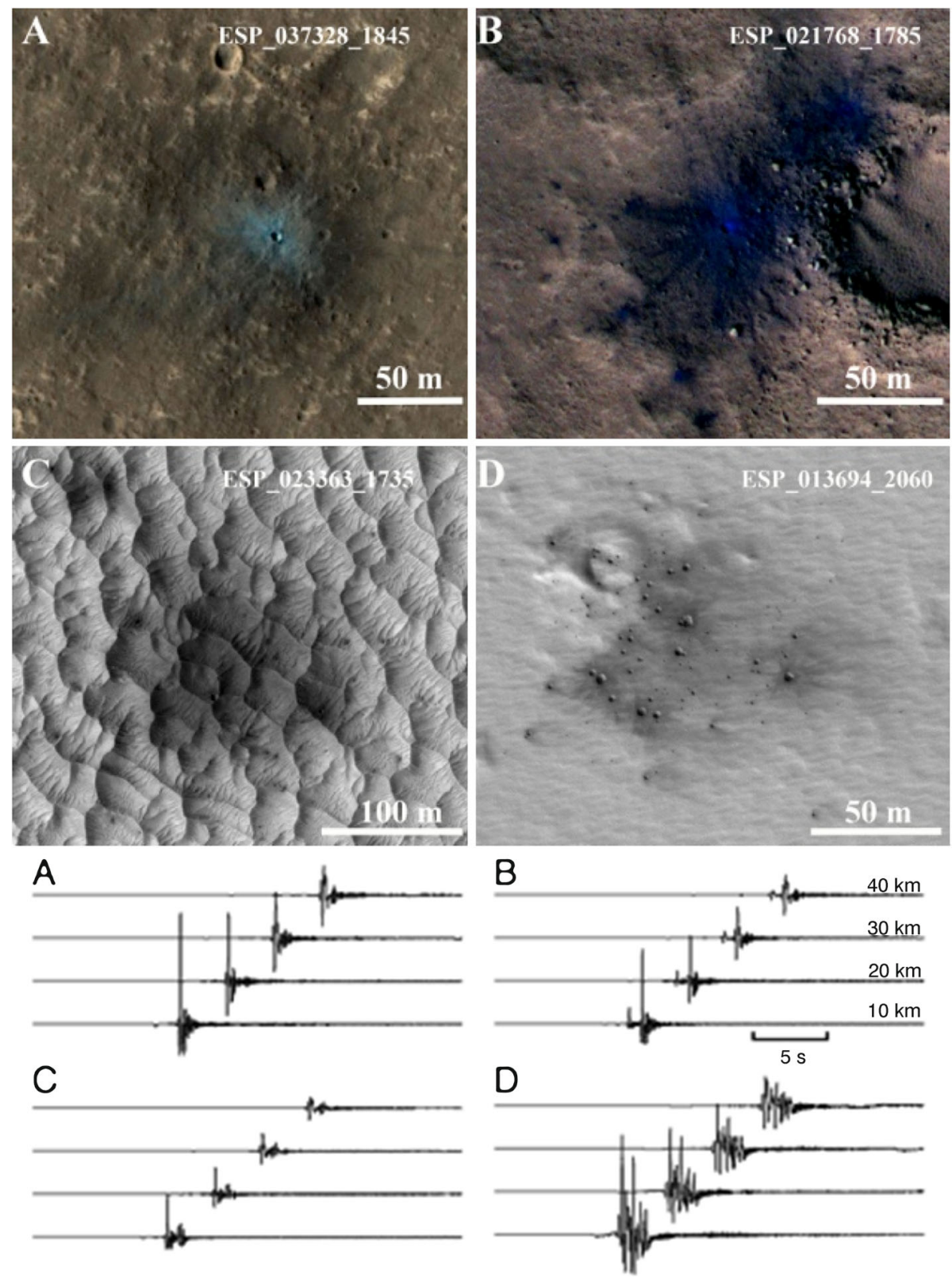

Fig. 20 Examples of new, dated impact sites with various numbers of individual craters: (A) single crater; (B) 3 craters; (C) 6 craters; (D) $>100$ craters. HiRISE observation IDs are indicated on images. For all: North is up; sun is roughly to the west. $\mathbf{A}$ and $\mathbf{B}$ are from enhanced false color RDRs; $\mathbf{C}$ and $\mathbf{D}$ are from red RDRs. Lower panels show vertical component synthetic seismograms for these distributions of craters at various distances, using the model of Schmerr et al. (2016) and an impact force transfer source. Clustered impacts are spread artificially over 2 seconds to simulate non-simultaneous impacts. This spread in time is longer than expected for most cases (should typically be $\ll 1$ second; Daubar et al. 2018), but is used as an extreme upper bound here for comparative purposes. Note that background noise is not included in this model, so the overall detectability of these events cannot be inferred from these plots. Image credit: NASA/JPL/University of Arizona (Banks et al. 2015; Schmerr et al. 2016) 


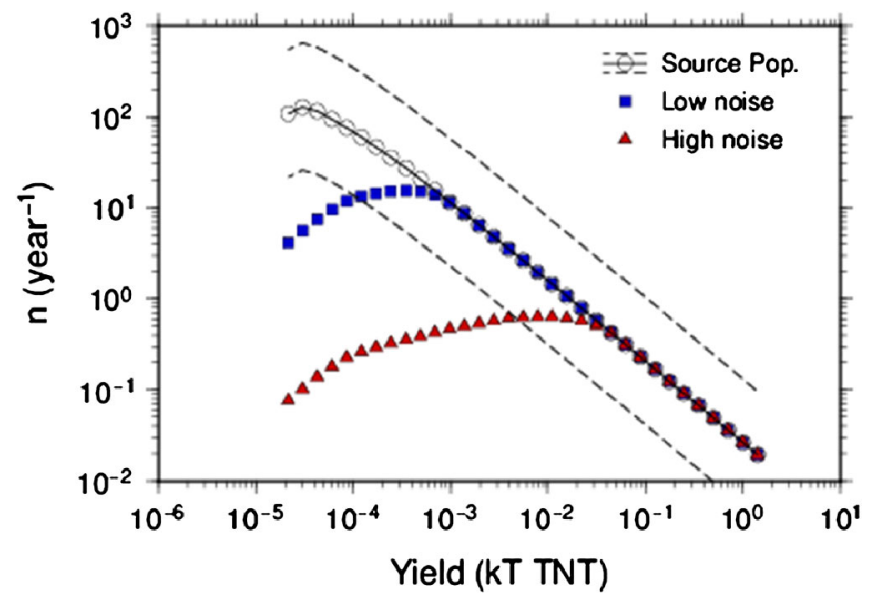

Fig. 21 Predicted airburst population and InSight detections. Based on observations of new impact craters by Daubar et al. (2013), Stevanović et al. (2017) estimated the number of events that would be seismically detectable to be 10 and 200 per year, integrated over the $\sqrt{2}$ incremental yield bins plotted here, for high and low noise cases respectively. This estimate contains an order of magnitude error, indicated by the dashed lines. Figure modified from Stevanović et al. (2017). Note that airbursts are only predicted to occur for yields between $2 \times 10^{-5}-2$ kiloTons TNT; larger events always penetrate the atmosphere and impact the surface, and smaller impactors are ablated or are slowed by drag to terminal velocity. Note these are based on the air-coupled seismic wave

some general characteristics of the impact and inform the orbital image search: what to look for and how detectable the impact will be in images. Overall, the seismic signal of more dispersed clusters will be less detectable than the impact of an intact bolide, and this will reduce the overall number of impacts InSight can expect to detect at Mars.

\subsubsection{Airbursts on Mars}

On the far end of the fragmentation spectrum lie airbursts. Surface effects of Martian airbursts have been observed in the form of thousands of small dust avalanches distributed asymmetrically around new dated craters (Burleigh et al. 2012). The number of airburst events that will be detected by InSight seismometers will depend on three main factors; the incident impactor population, the process of generating an airburst (which may be said in turn to depend on atmospheric and material properties), the Martian acoustic properties, and finally on the detection capability of SEIS.

The total overall incident bolide population at Mars is different from that at the Earth due to the proximity of the asteroid belt and Jupiter family comets. Other potentially impacting objects may be long-period comets sourced from the Oort cloud. By scaling the known size-frequency distribution (SFD) from Earth according to differences in impactor source population, planetary surface area and impact velocities, we can derive a flux SFD for Mars to be $\log _{10}(N)=a-b \oplus \log _{10} E$, where $N$ is the cumulative number of impactors per year incident on the Martian atmosphere of energy $E$ and $a$ and $b \oplus$ are empirically fitted constants (see Stevanović et al. 2017 for more details). This can be compared to observed current cratering SFD on Mars to verify the relationship (Malin et al. 2006; Daubar et al. 2013). Figure 21 shows the predicted airburst population on Mars along with predicted detection rates. Stevanović et al. (2017) predicted $\sim 10-200$ seismically detectable 

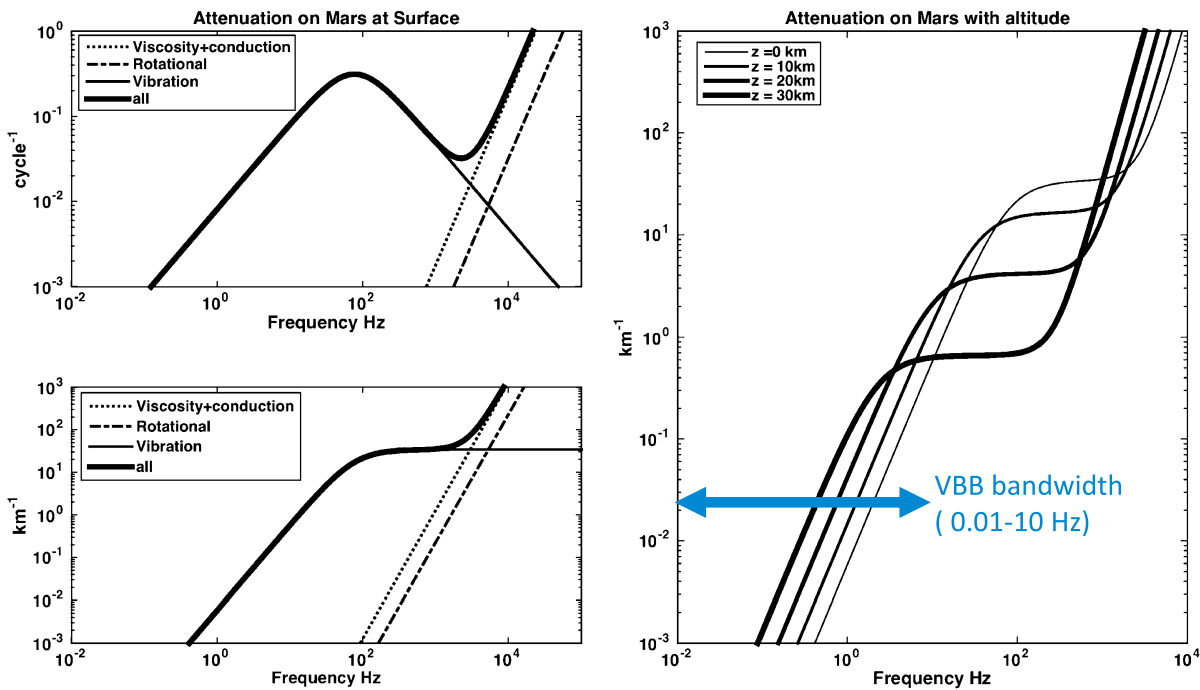

Fig. 22 Acoustic attenuation in the Martian atmosphere as a function of frequency. Left top: Attenuation per cycle. Left bottom: Attenuation factor in $\mathrm{km}^{-1}$. Attenuation due to atmospheric viscosity and conduction (dotted line), molecular rotation (dashed line), molecular vibration (thin solid line), and the sum of all sources (thick solid line). This illustrates that molecular relaxation has a major effect in the upper part of the bandwidth of the APSS sensor (e.g. above $1 \mathrm{~Hz}$ ) and is dominating attenuation; it causes almost 3 orders of magnitude more attenuation than atmospheric viscosity at these frequencies. Generally, from $1 \mathrm{~Hz}$ to $10 \mathrm{~Hz}$ the attenuation is significant, with attenuation lengths less than $100 \mathrm{~km}$ limiting likely detections of signals from purely atmospheric propagation to only those generated in the immediate region of the lander. Below $1 \mathrm{~Hz}$, remote detection will be possible, as there is much less attenuation. Right: Total attenuation factors at different altitudes (shown with different line thickness), showing attenuation is $\sim 20$ times larger at $30 \mathrm{~km}$ than at the surface

events, depending on the noise level of SEIS. This estimate contains an order of magnitude error resulting mainly from uncertainties in the air-ground coupling efficiency factor, atmospheric attenuation of the shockwave, amounts of seismic attenuation, and source population estimates. However, seismic signals from airbursts will allow detection of many more events than the generation of seismic waves by the impact to the surface alone.

Compared to Earth, very large differences in the acoustic attenuation occurs because of the $\mathrm{CO}_{2}$ composition of the Martian atmosphere. As pointed out by Bass and Chambers (2001) and Williams (2001), molecular relaxation is the largest source of attenuation for infrasound waves at Mars. This is in contrast to Earth, where this attenuation source can be neglected. This results in very large attenuation, as illustrated by Fig. 22, which shows the attenuation factor of acoustic waves as a function of both altitude and frequency. The attenuation factor is defined as the inverse of the distance over which the amplitude decays by $e$. At $5 \mathrm{~Hz}$, an attenuation factor of $\sim 1 \mathrm{~km}^{-1}$ will likely prevent remote observations of acoustic waves. At $1 \mathrm{~Hz}$, attenuation factors are $\sim 1 / 200 \mathrm{~km}^{-1}$, thus these frequencies will have more potential for regional airburst detections. Short period surface waves (5-10 s) will be weakly attenuated further.

The previously described modeling by Stevanović et al. (2017) shows that most airbursts occur at altitudes below $10 \mathrm{~km}$. Therefore, the final airburst will occur close enough to the ground that acoustic waves incident on the surface will only be moderately affected by atmospheric attenuation before they are converted into to seismic phases that will propagate through the planetary body to the seismometer. Stevanović et al. (2017) estimated this at- 
tenuation effect to be 0.7 for a moderate airburst and considered it negligible for the largest ones. Figure 22 shows that this is likely a reasonable assumption, at least in the VBB bandwidth, assuming the shock cone of the airburst is smaller than $1 \mathrm{~km}$, and the SEIS signal is recorded below $10 \mathrm{~Hz}$.

These phases will travel much more quickly than the airwave, so they are likely to be observed as precursor phases of the acoustic waves described in Sect. 4.5.3. Importantly, they are likely to have larger amplitudes than the seismic waves excited by the direct impact on the surface. See Sect. 6.2 for further discussion.

\subsubsection{Potential for Acoustic Wave Detection from Impacts on Mars}

As described in this paper, many meteorite atmospheric entries will produce surface impacts generating acoustic waves at the impact site. In most cases, the continuous sound speed decrease with altitude in the Martian atmosphere will not allow these acoustic waves to propagate back to the surface. However, various wind jets in the atmosphere may duct back these waves in specific directions (Garcia et al. 2017). Moreover, during the night, the surface temperature gradient generates a wave guide close to the surface that may allow detection of these acoustic signals far from the impact source (Garcia et al. 2017). The acoustic waves created by seismic waves following the impacts will also face similar propagation constraints. In addition, their amplitude is predicted to be much smaller than the acoustic waves created by the explosion, due to the large impedance contrast between the Martian ground and atmosphere (Lognonné et al. 2016). In the absence of positive identification of an impact (see Sect. 7.2), it will be challenging to definitively identify these signals as acoustic waves associated with an impact. InSight team members and associates are hopeful that direct acoustic signals from impacts will be detectable by the pressure sensor, if they are large enough and in band for the sensor, once the background noise level of the APSS sensors have been characterized.

\section{Benchmarking Impact Seismic Waveforms}

\subsection{Comparing Models of Synthetic Seismograms}

We performed a benchmarking study of different codes being used by members of the InSight team to compute synthetic seismograms. The primary objective of this study was to compare the results of the various methods in the case of modeling meteor impacts on Mars. This comparison leads to a cross-validation of the techniques and a better understanding of their respective advantages, limitations, and weaknesses. Secondly, the synthetics provided for the benchmark can be used as a catalogue to estimate detection thresholds and characterize impacts as seismic sources.

To obtain comparable results, we selected one of the InSight interior structure reference models (Panning et al. 2017; Smrekar et al. 2018). This is a realistic one-dimensional model of the interior structure of Mars, the EH45TcoldCrust1 model (Rivoldini et al. 2011). The density and velocity profiles of the model are shown in Fig. 23a. In the case of impacts, which are seismic sources occurring at the very surface of the planet, it is of major importance to consider the shallow interior structure. For this reason, we also used a modified version of EH45TcoldCrust1 that differs from the original in the top $1 \mathrm{~km}$. This modified model includes an $80 \mathrm{~m}$-deep layer of regolith and unconsolidated material overlying fractured bedrock (Fig. 23b). The regolith layer is characterized by low density and low seismic 

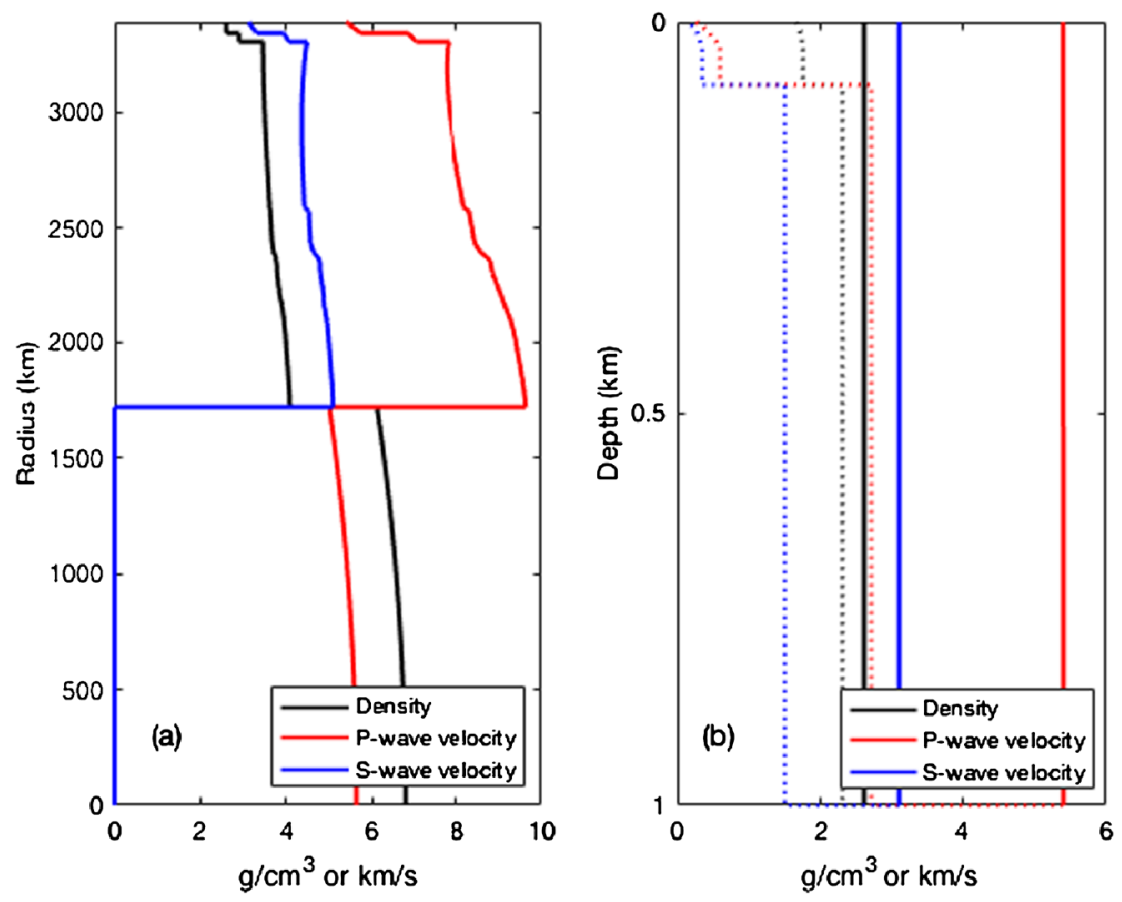

Fig. 23 Interior structure models used in the benchmark. (a) Density (black) and velocity profiles (red and blue for P-wave and S-wave, respectively) of the EH45TcoldCrust1 model (Rivoldini et al. 2011). (b) Zoom in on the upper $1 \mathrm{~km}$ of the EH45TcoldCrust 1 model (solid lines) and the modified version including fractured bedrock and regolith (dotted lines)

velocities, which can significantly modify the waveforms and amplitudes of seismic signals. Attenuation is also taken into account: we use a quality coefficient (a quantity that describes energy loss due to attenuation) for shear waves of 600 in the crust and 143 in the mantle. Bulk attenuation is neglected. In the regolith layer the quality coefficient increases linearly with depth from 100 to 300 over the first 80 m, as proposed by Morgan et al. (2018).

Two different seismic sources were used. The first was an impulsive explosion at the surface, described by a diagonal moment tensor with each component equal to $5 \times 10^{10} \mathrm{Nm}$. The second source was a vertical point force of $4 \times 10^{7} \mathrm{~N}$ applied at the surface. For both sources, a Dirac delta function was assumed for the source time function. These sources were selected to be representative of meteor impacts generating craters with diameters 25$40 \mathrm{~m}$ (Fig. 6). For both sources, synthetic seismograms were computed at epicentral distances of 50,100, 500 and $2000 \mathrm{~km}$, with and without the regolith layer.

Here we briefly describe the different codes used in the benchmark; for details, see the respective references. Minos is a normal-mode summation code based on the classical Mineos (Gilbert and Dziewonski (1975), updated by Woodhouse (1988) and rewritten by Masters) and developed as the 1D version of HOPT (Lognonné and Clévédé 2002; Clévédé and Lognonné 2003). Direct Solution Method (DSM) is a technique used to compute synthetic seismograms (Geller and Ohminato 1994; Geller and Takeuchi 1995), recently adapted to the case of Mars. Two versions of the DSM code were used in the tests, which were independently modified for Mars. These codes are denoted by DSM and DSMv2. DSM requires computation of high-angular order coefficients even for low fre- 
quencies when we need to calculate the seismograms near the surface (e.g. Kawai et al. 2006). DSM automatically truncates the angular order by measuring the convergence of coefficients at the surface and is efficiently parallelized for this purpose, but with a $0.1 \mathrm{~km}$ depth source. DSMv2 manually fixes the angular order and puts the source at $23 \mathrm{~km}$ depth. Herrmann's Computer Programs in Seismology (CPS, Herrmann 2013) is a package for the computation of synthetics in a flat, layered planet. AxiSEM (Nissen-Meyer et al. 2014) is a spectral-element based method allowing the computation of seismograms for axisymmetric models. GEMINI is a numerical method to compute ground motion through integration of an appropriate system of ordinary differential equations (Friederich and Dalkolmo 1995).

Not all the methods, however, were used for all computations. This depends on the characteristics of each technique and of the targeted synthetics. In particular, DSMv2 was used only for the model without regolith and for epicentral distances of $500 \mathrm{~km}$ or larger. More precisely, due to limits on computational run time, the synthetics were generated with a maximum of 18000 radial grid points ( $200 \mathrm{~m}$ spacing), which precluded resolving the $80 \mathrm{~m}$ regolith layer. In addition, convergence of the method was affected by the source depth, with shallower depths requiring computation to higher angular orders to reproduce the near field terms (see discussion in Kawai et al. 2006). For this reason, a source depth in the middle of the top layer was used (23 km depth) as a compromise. The far field body-wave wavefield is unaffected by this depth shift (see Teanby and Wookey 2011), and the synthetics beyond about $500 \mathrm{~km}$ converged. However, as a result of this non-zero depth the surface waves are not representative of an impact, and a small time lag correction is required. CPS, instead, was used only with modal summation, and therefore only surface waves were modeled. Although wavenumber integration can be used with this package, the required computation time would increase significantly. Finally, for the model with regolith, GEMINI exhibited numerical issues at short epicentral distances (50 and $100 \mathrm{~km}$ ) with unphysical wraparound phases.

The results for the explosion source and the structure model without regolith are shown in Fig. 24. The synthetic seismograms represent vertical ground velocity and are bandpass filtered between 0.05 and $0.2 \mathrm{~Hz}$ with a fifth-order Butterworth filter. Results for the radial component are not shown, but they are similar to the vertical case. The codes give very similar results in terms of amplitudes and waveforms at all epicentral distances, with a few exceptions. CPS was used to compute surface waves only, so no P-wave arrival is present. Moreover, at large epicentral distances (i.e. $2000 \mathrm{~km}$ ) a time shift appears relative to the other models, which is due to the equivalent flat planet used. As described above, DSMv2 used a source at depth and thus surface waves are significantly smaller; also, a difference in the P-wave arrival is produced and the synthetics were time-shifted by $3 \mathrm{~s}$. Finally, the GEMINI synthetics needed to be scaled in amplitude by a factor of two, which requires further investigation.

For the structure with regolith, we can still observe good agreement between DSM and AxiSEM compared to MINOS, which could have suffered from long-period noise before the first arrivals. CPS uses Earth flattening, so it is not surprising to have phase delays at large distances. Another observation on the comparison will be later phases calculated with Gemini. Since Gemini uses the strong form of equation of motion, whereas DSM and AxiSEM use the weak form, the treatment of boundary conditions can be ad-hoc (cf. Geller and Ohminato 1994; Komatitsch and Vilotte 1998). This will cause accumulation of numerical errors at some conditions. If we look at the frequency content, there are some significant discrepancies between Gemini and the pair of DSM and AxiSEM at certain frequencies. 

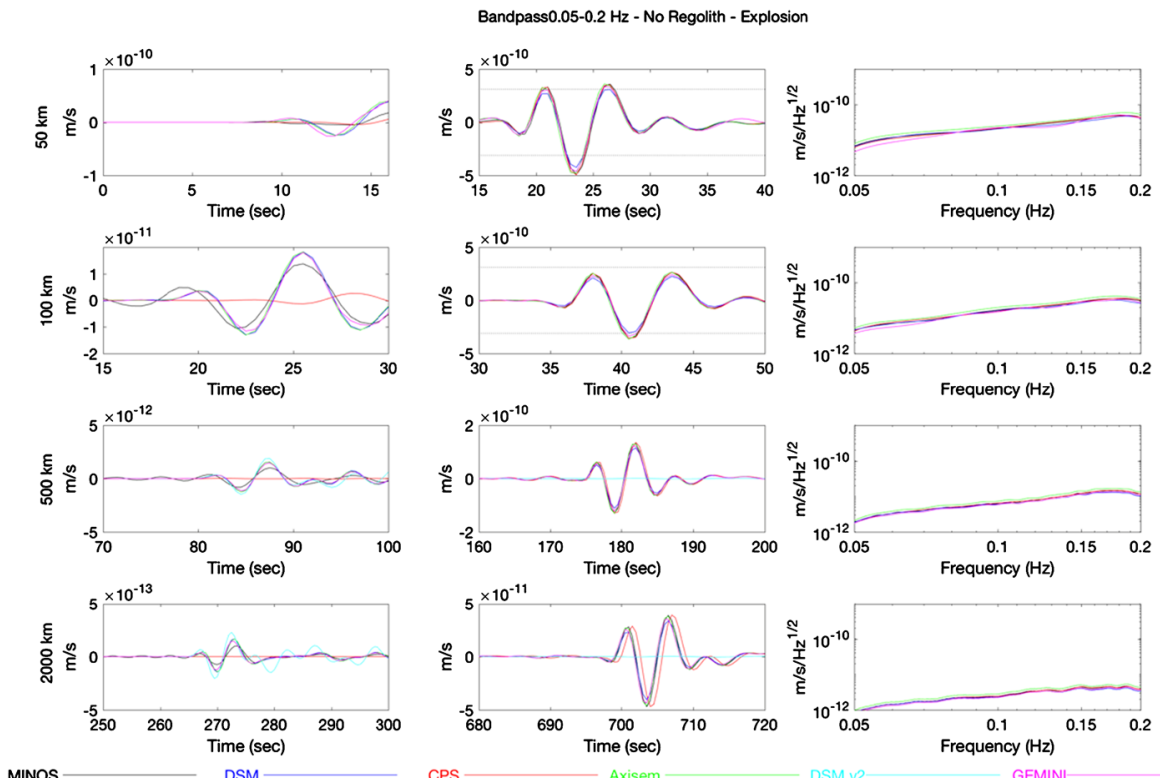

Fig. 24 Results of the benchmarking study for the explosive source and the original structure model without regolith, using six different techniques as described in the text. In each row, from left to right: zoom on the P-wave, zoom on the highest amplitude surface waves, and spectra. The rows are at increasing epicentral distances of 50,100, 500 and $2000 \mathrm{~km}$. All seismic data are in vertical velocity and bandpass filtered between 0.05 and $0.2 \mathrm{~Hz}$. The root-mean square noise, based on the InSight requirements, is represented by dashed lines whenever smaller than, or comparable to, the signal

We can explain this phenomenon by introducing optimal accuracy of numerical operators: numerical errors in operators will result in a large error only in the vicinity of the eigenfrequency of the mass and stiffness matrices, due to a zero division of the error propagator of the operator to the resulting waveforms (e.g. Geller and Takeuchi 1995).

For the structure model with regolith (Fig. 25), the agreement between the different techniques is still good for surface waves, especially for epicentral distances below $2000 \mathrm{~km}$. Body waves instead exhibit larger differences between the methods, which show the difficulties of accounting for this very-low velocity layer right below the surface. The fit for first $\mathrm{P}$-wave arrivals (time and amplitude) is, however, satisfactory. The case of the vertical point force gives analogous results (Figs. 26 and 27 for the model without and with regolith, respectively).

To summarize, this benchmarking study enables us to better understand the use of standard numerical methods to model the seismic signals generated by meteor impacts. In the simple example of a planet without regolith, all the techniques able to describe a surface source give very similar outputs up to $0.2 \mathrm{~Hz}$ in frequency. If this is interesting especially for surface waves, it should be noted that most of the body-wave energy is expected to be at higher frequency, above $1 \mathrm{~Hz}$. When using CPS, a more careful correction for the flattened models should be taken into account to avoid a small time-shift at large epicentral distances. The more realistic case with regolith is more complicated: the decay of the signal and the body-wave reverberations are not reproduced in exactly the same way by the different codes. However, the maximum amplitudes of the signals, as well as their arrival times, compare well. In this respect, it is interesting to note that, for the same source, amplitudes 


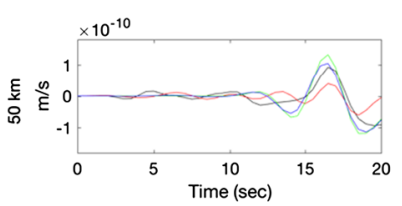

Bandpass0.05-0.2 Hz - Regolith - Explosion
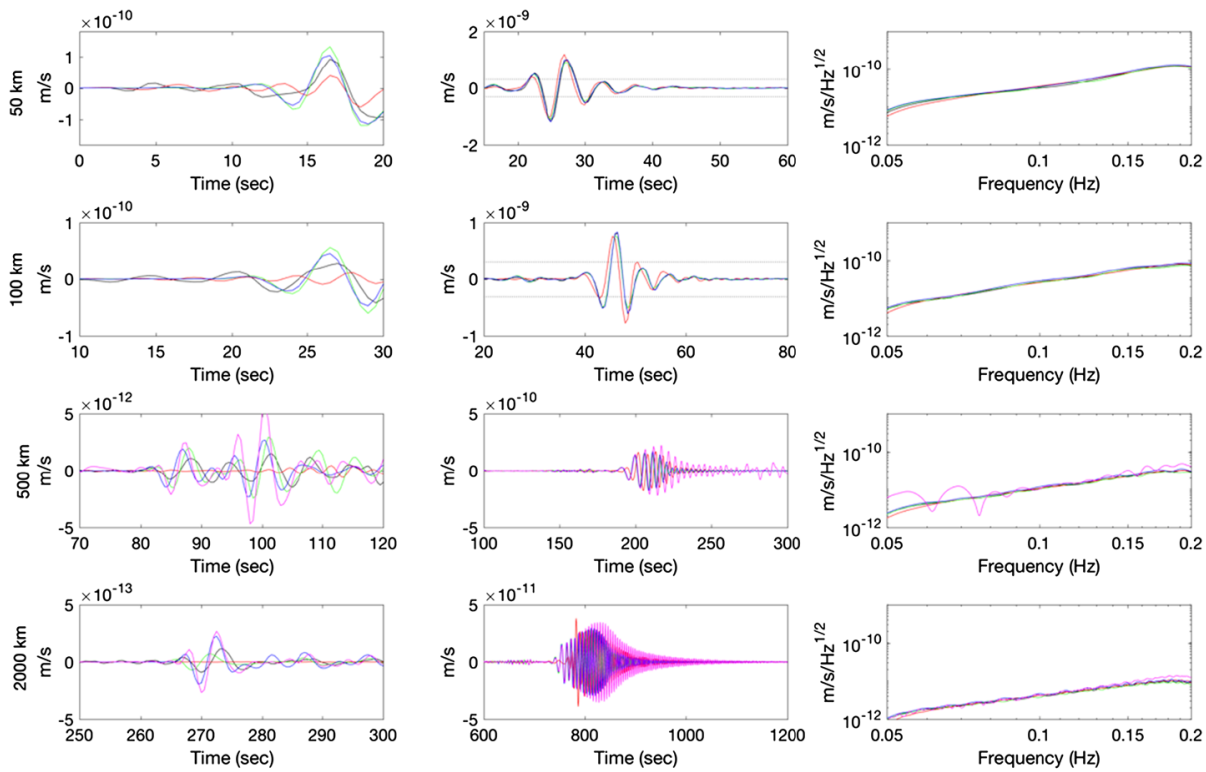

MINOS DSM

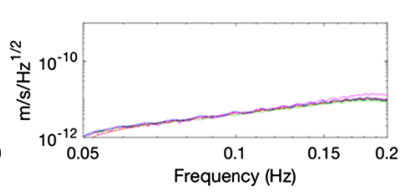

Fig. 25 Same as Fig. 24, using the modified structure model that includes a regolith layer

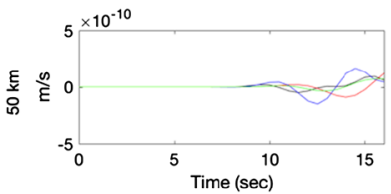

\section{Bandpass0.05-0.2 Hz - No Regolith - Point force}
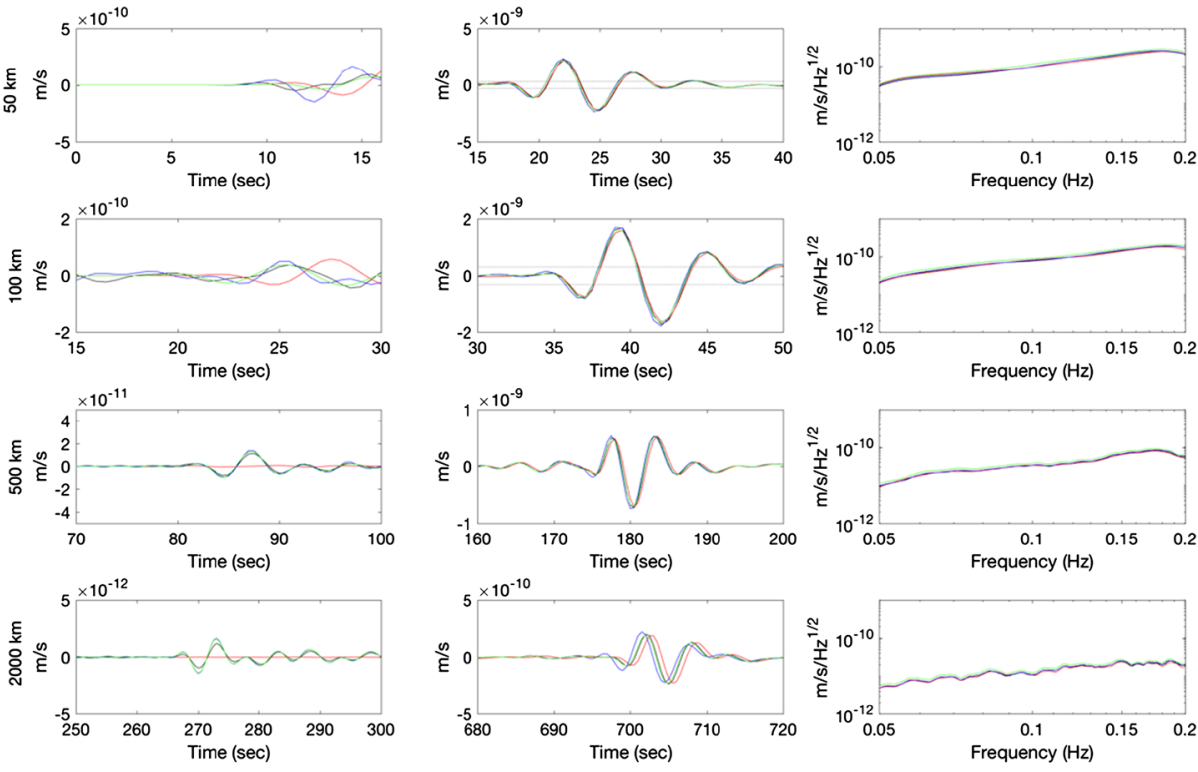

MINOS

CPS -

Axisem

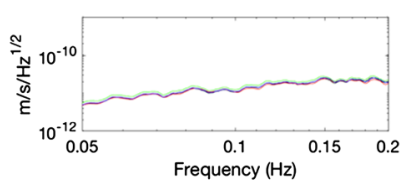

Fig. 26 Same as Fig. 24, but for a vertical point force 


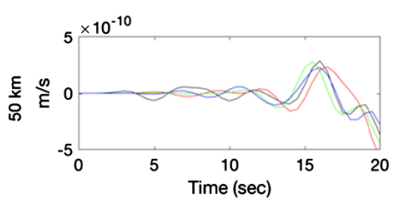

Bandpass $0.05-0.2 \mathrm{~Hz}$ - Regolith - Point Force
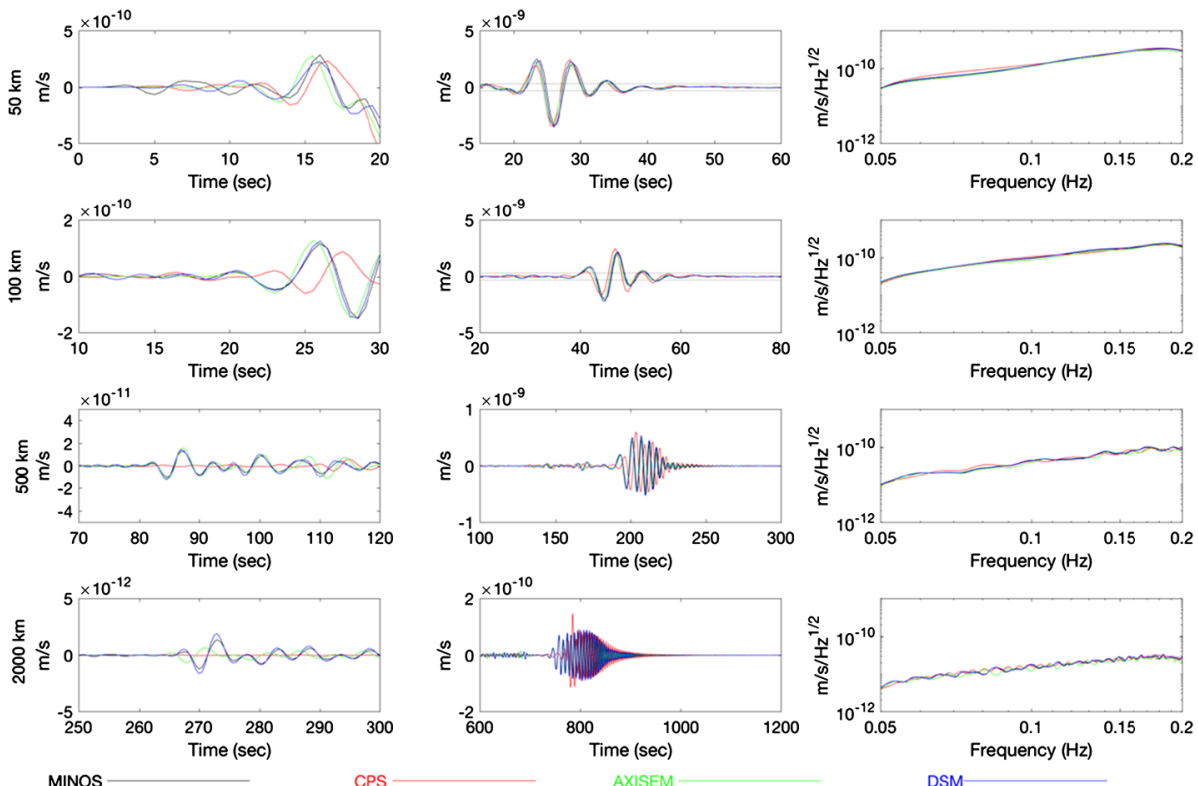

Fig. 27 Same as Fig. 24, but for a vertical point force and the structure model including a regolith layer

are larger in this case: the detection of impacts on Mars will most likely be possible thanks to the regolith layer and its behavior in terms of seismic energy conversion (see Sects. 3.1, 3.3 and 3.4 for more discussion).

\subsection{Seismic Amplitude as a Function of Distance}

The detectability of impacts on Mars is affected by the size of the source (source magnitude), the distance of the station from the source (geometric spreading), and the transmission properties of the Martian subsurface (intrinsic attenuation and seismic scattering). The seismic amplitudes from the impact itself are dependent upon the efficiency of momentum transfer in the impact, including the energy lost to damaging of the target materials, removal of ejecta, and heat, and efficiency of conversion of impact momentum into seismic ground motion (discussed in Sect. 3.3). For a given size impact source, we can estimate the seismic amplitude as a function of the epicentral distance of the source using a 1-D wave propagation simulation.

These synthetic wave propagation simulations require the assumption of a background structure; here we assume Model-A of Sohl and Spohn (1997) updated with the model from Rivoldini et al. (2011) and add a simple 1-layer crust of $50 \mathrm{~km}$ thickness, with a S-wave velocity of $3200 \mathrm{~m} / \mathrm{s}$ and P-wave velocity of $5000 \mathrm{~m} / \mathrm{s}$. We chose to keep this model simple as the details of the Martian interior are not yet constrained. We vary the attenuation structure within these models, assuming three background reference levels, high-Q $(Q=500)$, intermediate $(Q=100)$, and low-Q $(Q=50)$ to investigate the effect of attenuation structure on wave propagation and detectability (Fig. 28).

The highest amplitude waves produced in a seismic event are typically the surface waves. Surface waves don't show up in the lunar data owing to the high degree of scattering in 

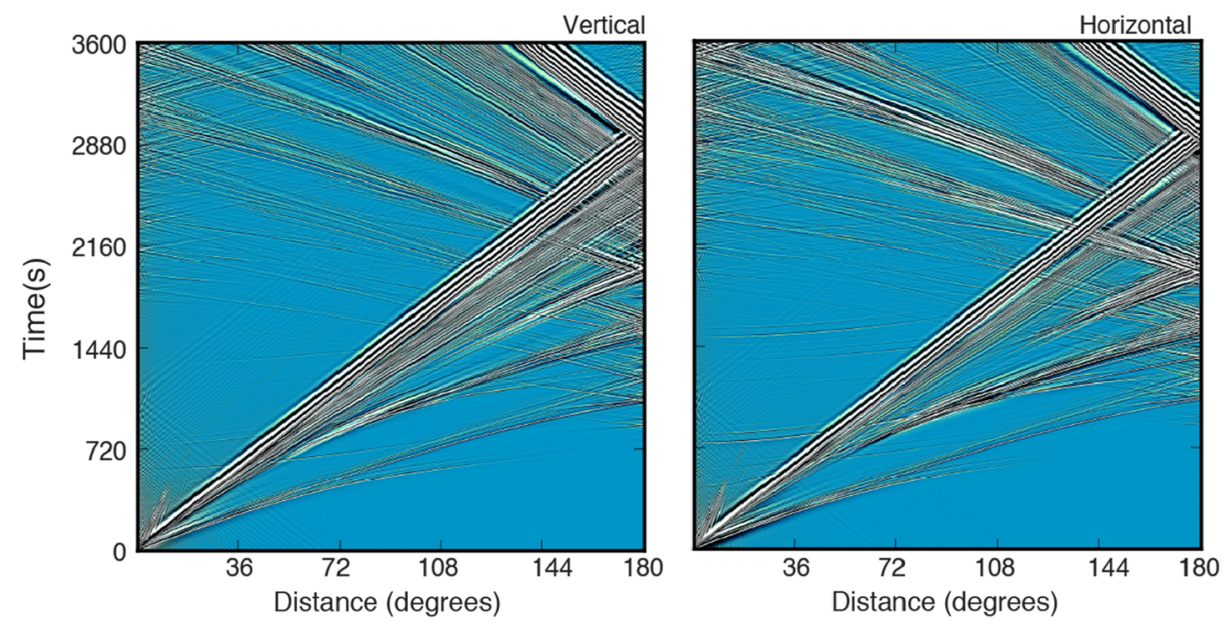

Fig. 28 1-D wave propagation simulation of impact energy propagating within the interior of Mars. Amplitudes are scaled to the peak ground motion in the time/distance window. Positive amplitudes are white, negative amplitudes black. Wave propagation is calculated using GEMINI (Friederich and Dalkolmo 1995), scaled in amplitude to match the amplitudes found with all other benchmarked modeling techniques

the lunar regolith and megaregolith (where they primarily propagate; see Sect. 2.2). Impact sources should generate Rayleigh waves through P-SV coupling (as demonstrated in the synthetics for an impact-like source), but not Love waves. The surface waves are quite susceptible to scattering and attenuation effects that are particularly strong near the surface, meaning they are lost more readily than the body waves that travel below the surface.

In our modeling, the highest amplitude waves produced by impacts are the surface waves. On Mars, it is an open issue how these surface waves will be affected by the scattering associated with crustal heterogeneities and impact-associated faults. If Mars is Moon-like, we can indeed expect the surface waves to be strongly affected by scattering and to have amplitudes significantly smaller than those modeled in 1D cases, as shown by modeling done by Gudkova et al. (2011). In addition to the poor long-period sensitivity of the Apollo seismometer when operating in the most used peaked mode, this led to no observations of surface waves on the Moon. On the other hand, observations of surface to near-surface explosions on the Earth allow the recording of both surface waves and body waves (e.g. Hedlin et al. 2002).

To determine the detectability of surface waves as a function of distance, we find their maximum amplitude occurring within one hour of the impact source. This is repeated for each epicentral distance and attenuation value. Here we assume a $10 \mathrm{~m}$ diameter craterforming impact as our reference source. In the near vicinity of the impact, ground acceleration is high and decays rapidly with distance from the source. At high frequencies $(1 \mathrm{~Hz})$ this effect is large (Fig. 29) with $1 \mathrm{~Hz}$ waves falling below the expected overall noise level at $15^{\circ}$ from the source for intermediate attenuation values $(Q=100)$. At longer periods, the waves from a $10 \mathrm{~m}$ diameter crater should propagate globally with a relatively high signal to noise ratio. For this reference source, the amplitude is below the noise requirement for an epicentral distance of $15^{\circ}$, or $\sim 900 \mathrm{~km}$. Within this distance from the landing site, we can expect reasonable homogeneities in the Martian crustal structure. The younger northern terrain, which might be less fractured than the lunar crust, might provide more Earth-like than Moon-like conditions for surface waves. Therefore surface wave detection from sources to the north may be more likely than on the Moon. 


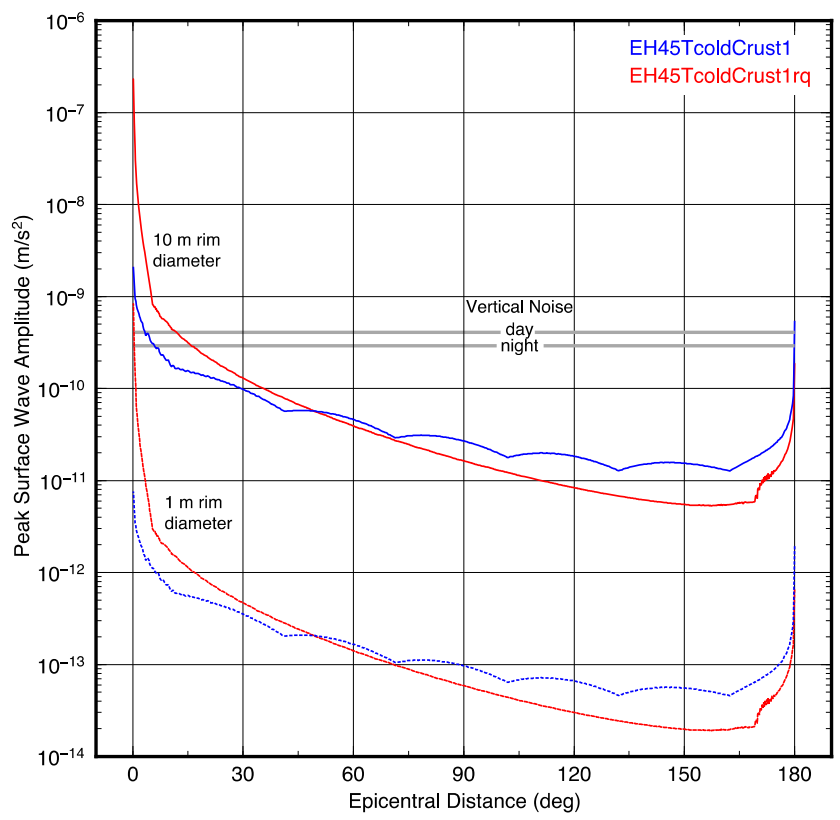

Fig. 29 Estimated seismic amplitudes from impacts and the sensitivity of the InSight SEIS-VBB to detecting waves generated for a seismic efficiency of 0.005 by (A) a $10 \mathrm{~m}$ diameter crater $($ moment $=$ $\left.1.922 \times 10^{10} \mathrm{Nm}\right)$, and $(B) 1 \mathrm{~m}$ diameter crater $\left(\right.$ moment $\left.=5.801 \times 10^{7} \mathrm{Nm}\right)$. Synthetics are generated using GEMINI (Friederich and Dalkolmo 1995), scaled in amplitude and corrected from surface amplification as explained in the text, for a $0 \mathrm{~km}$ explosive moment tensor source. The background models used are from Rivoldini et al. 2011 (described in Sect. 5.1). The seismic moment is calculated for each crater size using the crater scaling of (Teanby and Wookey 2011) and corrected for regolith effects using a scaling factor of 18.2 (as defined in Sect. 3.4, Eqs. (10) and (11), with values from Sect. 3.2). Data are bandpass filtered from 0.2 to $0.05 \mathrm{~Hz}$. We measure the peak amplitude of the Rayleigh wave using the first hour of the simulated seismogram after applying the bandpass filter. The expected diurnal variation in the SEIS-VBB noise floor for our frequency band is indicated in gray to indicate the detectability of the impacts (Mimoun et al. 2017)

\section{Impact Detections by Insight}

Recognizing impacts in the seismic data from InSight will be challenging at first. For one thing, empirical seismic recordings from terrestrial and lunar impact events are limited (Sect. 2). Another source of uncertainty is the largely unknown nature of the shallow and deep structure of Mars. With so many unknowns, we expect an exploratory period early in the mission, during which candidate possible impact signals will be identified based on various criteria. If several of these events can be confirmed to be impacts with orbital imaging of new craters (Sect. 7), the characteristics of impact-induced seismic signals will be better known, and identification and discrimination of these signals will become routine. Prior to data collection, we can plan on these various approaches to analyzing the data.

\subsection{Seismic Discriminators of Impacts}

Seismic signals from impacts differ in several important ways from interior, tectonic quake sources. An important feature of impacts is that they are exogenic, superficial events. This will be an important a priori constraint for the source location, as the depth is always near 
zero. Here we present several other features of seismic records such as this, which can be used to discriminate between tectonic and impact generated seismic events in the InSight SEIS data streams. This will no doubt evolve during the mission as our understanding of Mars and impact-generated seismic signals increases. To help with developing these impact diagnostics, we have drawn on the extensive work undertaken to monitor the nuclear test ban treaty. However, we note that most of the methods developed to discriminate nuclear explosions from earthquakes rely on a global network of seismometers, dense arrays, and infrasound detectors. With InSight, we will be limited to a single seismic station, necessitating a different strategy.

We have developed the following set of diagnostics that can be used to reject the hypothesis of an impact. These will be used in operations to reduce the number of candidate impact events for further analysis, event data requests, and orbital image crater searches. These diagnostics are based on first principles, explosive analogs, and lunar impacts.

Diagnostics to reject an impact hypothesis:

- First motion: An impact event will create a positive pressure impulse at the source, which will result in a positive first motion (away from the source) for the P-wave. Therefore, in principle, a negative first motion can be used to rule out an impact event. However, in practice, this is unlikely to be effective. Even on the Earth, where there are typically many stations available at various distances, this is considered unreliable because seismic noise can obscure the very first arrival, and so the direction of motion can be wrongly identified. Also, earthquakes or marsquakes can produce either a positive or negative first motion depending on the source mechanism as well as the back-azimuth and take-of angle defined by the source / station geometry and structure.

- $S$ wave energy: Impacts are likely to produce stronger P-waves relative to S-waves when compared to tectonic events, so high S-wave energy could be used to reject an impact source. However, the $\mathrm{P} / \mathrm{S}$ amplitude ratio is also a strong function of fault orientation and source/ station geometry, which will introduce uncertainty in this diagnostic.

- Magnitude ratio: On Earth, one of the most reliable diagnostics for explosive versus natural sources is comparing the body wave magnitude, $m_{b}$, to the surface wave magnitude, $M_{s}$. An earthquake (or marsquake) will produce more surface waves than an explosion (or impact). Therefore, a plot of $M_{s}$ versus $m_{b}$ can potentially be used to diagnose source type. Unfortunately, body wave magnitude will be difficult to estimate accurately from a single station due to the radiation pattern effect.

- Frequency content: Impacts and quakes clearly differ in terms of their source mechanisms. Quakes, which commonly occur as slip on a fault, are typically expressed as a double coupled force, while impacts are better explained with a single force (Sect. 3.1). This results in different frequency content of the seismic signal (Sect. 3.2). The source time function of faults is expressed with a step function. The spectrum is flat up to a certain corner frequency and then rolls off above the corner frequency. The spectrum is commonly expressed using 2-model, which the spectral power decay with power of -2 (e.g. Aki and Richards 2002). The model well explains terrestrial quakes as well as deep moonquakes (Aki and Richards 2002; Kawamura et al. 2017). On the other hand, Sect. 3.1 shows that source time functions of impacts, either from the GL or SWH models, are expected to be either derivative or with a high frequency overshoot. This difference in the seismic spectra is shown in Fig. 7. The spectrum of a quake is flat at low frequencies, similar to those with $B=0$, while that of an impact has an increase in the power in $\sim 1-2 \mathrm{~Hz}$. Figure 4 also shows an example of spectra from shallow moonquakes and impacts, showing the much smaller cutoff frequency of the impact spectrum compared to the quake. If these 
characteristic spectral features can be observed in the data, we can discriminate impacts from quakes through spectral analyses as we are locating the source.

- Depth phases: For deep marsquakes, in addition to the direct wave, there should be reflections from the underside of the surface that are sufficiently separated in time to be identified. For example, the $\mathrm{P}$ phase will be followed by the $\mathrm{pP}$ phase. If these phases can be identified in an event, then an impact source can be rejected.

It should be noted these discriminating criteria can be effective if Martian seismograms prove to be impulsive, like on Earth. If we observe more Moon-like seismograms (Sect. 2.2, Fig. 3), where scattering in the regolith produces very emergent long duration signals, it is highly unlikely any discriminator that relies on clear phase identification can be used. This only leaves the frequency content analysis (Fig. 4).

When applying these criteria, the usefulness of requested high frequency "event data" in addition to the continuous 2 samples/sec data (Sect. 7.1) will depend largely on the event size. For very large distant impacts, the continuous data should be adequate, as phases will be well separated and frequency content would be quite low (higher frequencies will be attenuated). In any case, such a large signal would no doubt be prioritized highly for downlink of event data, whether it was thought to be a quake or impact. For the more numerous regional events ( $<1000 \mathrm{~km}$ range), event data would be needed. The most diagnostic positive trait is likely to be the frequency content. This is likely to be $>1 \mathrm{~Hz}$ for small events, so event data would be necessary.

With only a single station on Mars, each of these diagnostics alone will have limited use, but by combining multiple diagnostics, many candidate impact events should be able to be rejected. Also, once a substantial catalog of marsquakes and impacts has been built up, some of the uncertainty associated with the fault double couple radiation pattern orientation could be mitigated if the event can be located and some estimate of regional stress could be incorporated to predict the mostly likely fault strike orientation. These diagnostics will naturally be refined during the mission, as more is learned about the seismic characteristics of a Mars impact.

Once a seismic event is determined to be a candidate impact based on these diagnostics, an estimate of its location will be necessary to find it on the surface. The Marsquake Service (MQS) will determine, whenever possible, locations and sizes of meteorite impacts from the seismic signals by applying methodologies and magnitude scales developed by Böse et al. (2017) and Böse et al. (2018, in press). Locations will be determined using independent approaches for distance and azimuth which are subsequently combined. Distance estimates include methods that use (1) identified body and surface wave phases and (2) multi-orbit surface waves. The latter will only be available for the largest events, and hence will almost certainly not be used for impact events. Errors can be included in the single-station event body phase-based distance estimates, as there are challenges in correctly identifying seismic phases, and there are significant model uncertainties. Additional errors stem from pick uncertainties. Wrong phase identification can lead to large errors in locations that are difficult to quantify and are typically not included the location uncertainty. The probabilistic framework of Böse et al. (2017) quantifies the remaining uncertainties as probability density functions. The key distinguishing features of impacts will be their spectral content and their shallow depth. It is extremely challenging to constrain event depth at distance using a single station, but a general indication can be provided by comparing the relative amplitudes of body and surface waves (Böse et al. 2018, in press). As discussed above, crustal reflection/depth phases play a critical role in constraining event depth, and these markers will be identified if possible. 
Preliminary tests (Böse et al. 2017) indicate that the errors in the estimated event locations are small enough to meet the Level 1 requirements of the InSight mission, if multiple clear body and surface phases are identified. These requirements specify that epicentral distances and back azimuths are to be determined to accuracies of $\pm 25 \%$ and $\pm 20^{\circ}$, respectively (Banerdt et al. 2013). Very large (and thus very rare) impacts that generate identifiable multi-orbit surface waves could result in location accuracies as small as $1^{\circ}(60 \mathrm{~km})$ in distance and $10^{\circ}$ in azimuth (Panning et al. 2015); however, this size impact is exceedingly unlikely to be seen by InSight. The successful identification and location of meteorite impacts in orbital images is crucial to generate ground truth locations that will strongly constrain structural models of Mars. Approximate locations of suspected meteorite impacts will be used as targets for the collection of high-resolution orbital images to enable visual identification and determination of exact impact locations (Sect. 7). The iterative refinement of Mars interior models with every meteorite impact and marsquake observed during the InSight mission will lead to improved event locations and reduced uncertainties (Khan et al. 2016).

Airbursts will be even more challenging to detect in seismic signals. When recorded at a seismic station, the most distinctive feature of an airburst is the arrival of the acoustic airwave. To distinguish an airwave arrival from other parts of the coda, it is necessary to examine the group velocity of the arrival. This should correspond to the local atmospheric sound speed. One potential difference between detection of an airwave on the Earth and Mars is the higher rate of attenuation in the Martian atmosphere, which may mean that it is difficult to detect this signal over large distances (Sect. 4.5.2). It is therefore imperative that the seismically coupled energy is well understood. If the airburst is large enough, acoustic energy will couple into the ground and propagate as seismic waves. These will be recorded as precursor signals before the arrival of the direct airwave. This air-to-ground coupling may produce an emergent waveform, due to the nature of the coupling along an extended raypath and not simply a point source. The precursor seismic signals are subject to all of the same principles as impacts, because acoustic-to-seismic coupling will have a similar effect as a direct surface impact. Further discussion of likely airburst characteristics can be found in Stevanović et al. (2017).

To detect acoustic waves from impacts, we will examine data from the pressure sensor data on InSight. The pressure sensor will be continuously sampled at 20 samples per second (SPS), and its instrument response should cover the infrasonic frequency range. The sensor will have good response to signals $\lesssim 5 \mathrm{~Hz}$. The sampling limits it (with Nyquist sampling) to $<10 \mathrm{~Hz}$. The plumbing on the inlet, and a low-pass filter in the sensor electronics, both limit it to $\lesssim 5 \mathrm{~Hz}$. We were unable to verify this in the laboratory, as the calibration system only successfully modulated the tested pressures at up to $\sim 1 \mathrm{~Hz}$. The precise cutoff frequency will be assessed after landing. Consequently, this sensor may detect acoustic waves created by impacts. However, only data at 2 SPS will be sent back to Earth continuously. To monitor pressure signals at frequencies above $1 \mathrm{~Hz}$, the energy of pressure variations in the $1-10 \mathrm{~Hz}$ frequency range will be computed on the lander and sent back to Earth at 1 SPS. This energy channel, named ESTA for Energy Short Term Average, will be analyzed by the science team to detect high frequency infrasound signals. Then, a request for high rate data will be sent to the lander to recover the time windows containing candidate infrasound events.

\subsection{Expected Frequency of Seismic Impact Detections}

The frequency of impact seismic signals InSight will detect is based on several factors: the incipient bombardment rate (Sect. 4.1); the efficiency of partitioning the impact energy of 
Table 3 Distance at which an impact forming a crater of a given diameter is estimated to be detectable by SEIS, using two different methods of estimation. These preliminary estimates are dependent on various unknown parameters such as the noise levels of the SEIS instrument, seismic efficiency, and attenuation in the Martian interior

\begin{tabular}{lll}
\hline Crater diameter $(\mathrm{m})$ & $\begin{array}{l}\text { Distance }(\mathrm{km}) \\
\text { (Teanby 2015) }\end{array}$ & $\begin{array}{l}\text { Distance (km) } \\
\text { (Daubar et al. 2015) }\end{array}$ \\
\hline
\end{tabular}

\begin{tabular}{rrr}
\hline 1 & 61 & 10 \\
5 & 295 & 100 \\
10 & 580 & 500 \\
15 & 862 & 1000 \\
20 & 1141 & 1400 \\
25 & 1419 & 1800 \\
30 & 1696 & 2100 \\
35 & 1971 & 2523 \\
40 & 2246 & 2909 \\
45 & 2519 & 3296 \\
50 & 2792 & 3682 \\
\hline
\end{tabular}

Fig. 30 Distance at which an impact forming a crater of a given diameter is estimated to be detectable by SEIS, using two different methods of estimation. See text for details about the two methods. These preliminary estimates are dependent on various unknown parameters such as the noise levels of the SEIS instrument, seismic efficiency, and attenuation in the Martian interior, so have large uncertainties

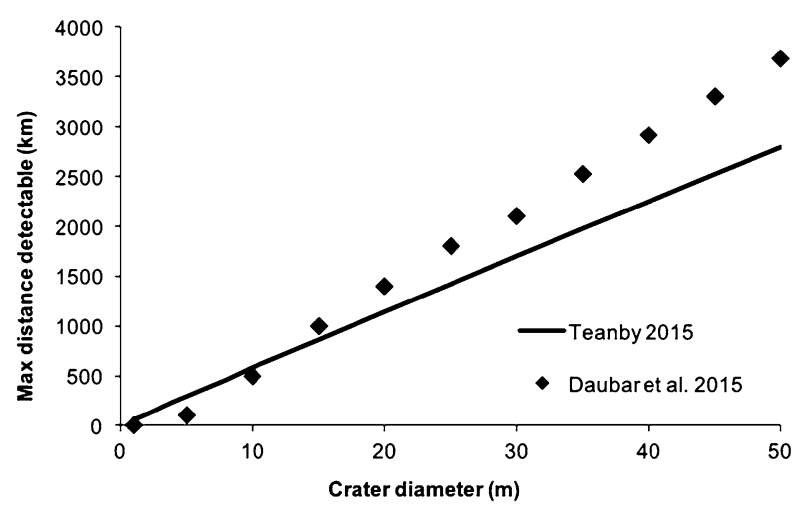

those impacts into seismic energy (Sect. 3.3); the nature of an impact's source time function (Sect. 3.1); propagation effects between the impact and the SEIS location and associated amplitude reduction due to geometrical spreading, attenuation, and scattering; and, last but not least, the amplitude of the resulting signals compared to the noise level of SEIS (Sect. 5.2). Large uncertainties on all of these factors makes it very difficult to determine the efficacy of InSight's monitoring of natural impacts. However, general trends can be predicted. For example, the larger the impact, the farther away it will be able to be detected. Using an overall impact rate and taking these factors into account, a detection rate can be estimated.

Teanby (2015) and Daubar et al. (2015) use independent approaches to estimate the relationship between seismic detectability and crater size. Teanby (2015) use empirical scaling laws based on lunar/terrestrial impacts, missile tests, and explosions to determine a relation between impact energy and seismic amplitude as a function of distance. Daubar et al. (2015) use estimation of the amplitude from Apollo impact observations, corrected for a priori differences between Mars and the Moon. See Lognonné and Johnson (2015) for details. The predictions of the two methods are compared in Table 3 and Fig. 30. These two approaches differ from the modeling hypothesis. These preliminary estimates are dependent on various unknown parameters such as the noise levels of the SEIS instrument, seismic efficiency, and attenuation in the Martian interior, so have large uncertainties. In any case, small impacts 


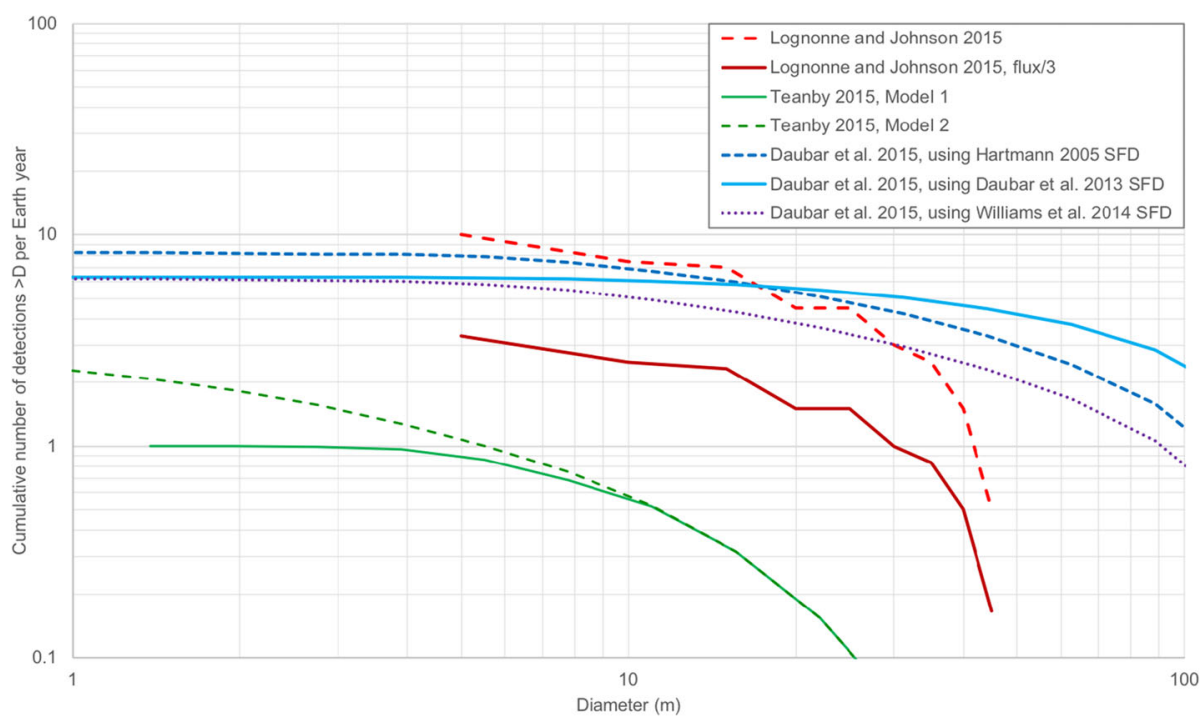

Fig. 31 Predicted number of cumulative SEIS impact detections per Earth year for a given crater diameter, made using various models and published production functions (size frequency distribution; SFD) to estimate the current impact rate. The Teanby (2015) model is for the SP (short period) sensors in SEIS, which has a sensitivity to impacts approximately eight times lower than the VBB (Very Broad Band) sensors, which the other models use. All of these estimates have an order of magnitude uncertainty. See text for more details

will only be detectable within a very limited range of the InSight landing site. Only impacts producing craters $\gtrsim 30-40 \mathrm{~m}$ in diameter will be detected at very far distances.

When the dependence between size and distance for detectable impacts (Fig. 30) is combined with the best measurements of the current impact rate (Sect. 4.1), we can calculate an overall estimate of the number of impacts detectable by SEIS per year (Fig. 31). Several estimates of this rate have been published (Davis 1993; Teanby and Wookey 2011; Lognonné and Johnson 2015; Teanby 2015; Daubar et al. 2015). Results are shown in Fig. 31 for cumulative impact detection rate per year for various of these models. Two factors balance each other out in the calculation of total detections. Many small impacts are occurring on Mars, but the detection distance is the limiting factor. There is very low likelihood that even a small impact will occur very close to InSight. The chances are also low of forming a crater large enough to detect even at great distances. In the last decade of monitoring the dusty areas of Mars, only a few craters have been observed to form that are larger than $30 \mathrm{~m}$ in diameter; the largest new impact to be found with before and after images thus far is $60 \mathrm{~m}$. However, these observations are limited to dusty areas, and require multiple images spaced in time to capture the event. The Hartmann and Daubar (2017) production function predicts $\sim 6$ craters larger than $30 \mathrm{~m}$ occur somewhere on the entire planet Mars each Earth year, but not all of those are observed in orbital images.

The models shown here differ in several ways. Lognonné and Johnson (2015) used data from the Apollo Network (Lognonné et al. 2009) to calculate impact amplitudes as function of the impact momentum and distance to station. They then corrected these amplitudes for the difference in seismic attenuation between Mars and the Moon, noting however that the latter is not major, as the source cutoff of impacts is likely the major frequency cutoff for impacts recorded at several thousand kilometers. Detections were then modeled with MonteCarlo simulations using the impact flux of Le Feuvre and Wieczorek (2011). Both Teanby 
and Wookey (2011) and Daubar et al. (2015) used the impact flux based on the recently occurring impacts observed by MRO. This flux has been discussed in Sect. 4.1 and is approximately three times smaller than that of Le Feuvre and Wieczorek for the size impactors generating observable signals. For this reason, the Lognonné and Johnson (2015) results are also shown in Fig. 31 divided by a factor of three to correct for that lower observed rate.

Daubar et al. (2015) used the same relationship between momentum and observed seismic amplitude as Lognonné and Johnson (2015), but used different published size frequency distribution (SFD) models of the impact rate. In contrast, Teanby and Wookey modeled the seismic waves using the Direct Solution Method and then estimated the amplitude of seismic waves on the seismic efficiency figure. Based on this measured rate of impacts, Teanby and Wookey (2011) predict a total impact-induced seismicity of Mars of $10^{13}-10^{14} \mathrm{Nm}$ per year. Teanby (2015) extrapolated this down to smaller impacts, which have not been observed from orbit, but that may be detectable seismically (their Model 2). Another difference between the Teanby (2015) model and the other two sets of models is that Teanby (2015) used a noise level of $10^{-8} \mathrm{~m} / \mathrm{s}^{2} / \mathrm{sqrt}(\mathrm{Hz})$, which is a conservative value appropriate for the SP (short-period) sensors in SEIS. The Lognonné and Johnson (2015) and Daubar et al. (2015) models use predicted noise limits for the VBB (Very Broad Band) sensors. At these frequencies, $\sim 0.5-\sim 2-3 \mathrm{~Hz}$, the VBB is a factor of $\sim 10$ better than the SP in detected amplitude and therefore in detected seismic moment (Mimoun et al. 2017). Thus the VBB may detect $\sim 8$ times more impacts than the SP. However, the highest frequencies from these small events will be above $1 \mathrm{~Hz}$, which is approaching the higher ambient/instrument noise crossover. Explosion/impact data from Teanby (2015) had peak frequencies $\sim 1-16 \mathrm{~Hz}$. The upper end of this range is not critical, as most of the data had peaks in the $1-4 \mathrm{~Hz}$ range (e.g. the Apollo impacts $\sim 2 \mathrm{~Hz}$; Fig. 4). So some degree of enhanced detection from the VBB over the SP is expected, but drastically lower noise levels may not be achievable for frequencies $\sim 1-2 \mathrm{~Hz}$. For the ambient noise, this could be challenging.

For this and other reasons, the resulting overall estimates of seismic impact detections (Fig. 31) are uncertain to several orders of magnitude because of the undetermined seismic properties of Mars such as attenuation, seismic coupling efficiency, and uncertainty in the current impact rate itself. Additionally, although the noise levels of SEIS have been modeled (Murdoch et al. 2017; Mimoun et al. 2017) and tested on the Earth to verify the required noise levels will be met, the true noise of the system will not be known until the seismometer is deployed on the surface of Mars. Given those uncertainties, Teanby (2015) estimates somewhere between $\sim 0.1-30$ impacts per Earth year will be detectable at moderate distances of less than $\sim 1000 \mathrm{~km}$. Lognonné and Johnson (2015) predicted $\sim 10$ impacts per year using the impact flux of Le Feuvre and Wieczorek (2011), which would be reduced to $\sim 3$ per year when using the latest constraints on the impactor flux. For very large events that could be detected globally, Teanby and Wookey (2011) estimate these occur only once every 1 to 10 years. Daubar et al. (2015) derived a similar estimate of $\sim 4-8$ total impacts would be detected per Earth year $(\sim 8-16$ in the primary InSight mission).

It should be noted that all of these estimates assume single-crater, unfragmented impactors. Atmospheric fragmentation leading to clusters of impacts will affect the seismic detectability of approximately half of current Martian impactors (Daubar et al. 2018; Schmerr et al. 2016) (Sect. 4.5).

Another factor that will reduce the number of detections is the low seismic moment associated with small impacts, and the fact that their high frequency energy is still limited by the source cutoff, a few $\mathrm{Hz}$ for the smallest detected by Apollo (Fig. 8). Scaling laws (Fig. 6) predict that the detectability of an impact drops by a factor of $10^{2.5}-10^{3}$ for every order of magnitude drop in crater diameter. Even this detectability assumes a relatively 
quiet background; the Martian environment is contaminated by abundant wind noise in the $10^{-6} \mathrm{~m} / \mathrm{s}^{2}$ amplitude range as detected by Viking 2 on the lander deck (Anderson et al. 1976; Nakamura and Anderson 1979). However, this noise level is three orders of magnitude larger than the expected InSight noise level at $1 \mathrm{~Hz}$ (Mimoun et al. 2017), so Viking's non-detection is easy to understand. For InSight, noise may be even lower than the requirement during the relatively quiet nights. Thus impacts generating smaller craters could be detected by InSight if they occur nearby, during periods of low wind activity, or in the night time.

\section{Operational Plans}

\subsection{Role of Impacts Science Theme Group}

The Impacts Science Theme Group (STG) has two main tasks: to coordinate scientific analyses by the InSight team related to impact cratering; and to ensure sufficient and appropriate data are acquired during the mission to perform those analyses. For the latter task, the Impacts STG will support surface operations of the InSight mission by participating in the science planning process. In the science monitoring phase, these operations are on a weekly cycle that is mainly focused on prioritizing downlink of high temporal resolution SEIS event data. The full operational process is described in Banerdt et al. (2018, this issue). The Impacts STG will be made aware of potential impact detections via the Mars Quake Service (MQS, Clinton et al. 2018). Relative prioritization among candidate impact events will be made at a weekly Impacts STG telecon prior to the Event Selection meeting. The Impacts STG will then send a representative to the Event Selection meeting to advocate for our highest priority event data. On a more long-term strategic timeline, the Impacts STG will have a representative at the Science Operations Working Group (SOWG) meetings. The Impact theme group's weekly telecons will also be used to organize and prioritize orbital image requests and collaborate on ongoing research activities.

Certain scientific investigations are desirable for impact science, but they are not part of the baseline mission plan of operations. For example, imaging at night to search for meteors as described in Sect. 7.2 will require additional planning and resources. The Impacts STG will seek approval for special activities such as these via Science Activity Requests. These requests will be prioritized by the science team and, based on those priorities, inserted into the tactical planning process.

During normal operations, the Impacts STG will prioritize event data for candidate impact events. Data acquired by SEIS is stored and processed by the flight software on board InSight. Two types of data are treated differently for downlinking:

(1) Continuous data are low temporal resolution (i.e. decimated) (2 samples/sec) data processed and downlinked daily with no time gaps within the data.

(2) Event data are full-resolution raw scientific data acquired and filtered from the instrument. Time segments of this full-rate data can be extracted, filtered, compressed, and then downloaded on request. Those segments are called event data.

Because the high-frequency SEIS data cannot all be downlinked due to data volume limitations, individual events must be identified in the lower resolution continuous data and prioritized for high-frequency event data retrieval; high-frequency SEIS data is stored on the spacecraft for approximately one month before it is overwritten. The STGs will prioritize this high-frequency event data for downlink within the data volume constraints each week. 
During routine operations, the SOWG (Science Operations Working Group) and the APAM (Activity Plan Approval Meeting) meetings lead to the definition of an Activity Plan containing placeholders for Event Requests. Those placeholders are filled with ERPs (Event Request Proposals) submitted by the Science team during the week. Any scientist can submit an ERP that will be reviewed and ranked among others during the Event Selection Meeting.

The Event Selection Meeting is led by the long-term planner (LTP) and chaired by the SEIS and mission PIs. Participants include PIs from SEIS, Temperature and Wind for InSight (TWINS), IFG (InSight Fluxgate), and PS (Pressure Sensor), STG leads pertinent to event selection, representatives from MQS (Marsquake Service), MWS (Mars Weather Services), SEIS community, and public outreach. See Banerdt et al. (2018, this issue) and Lognonné et al. (2018, this issue) for more details on these operational meetings. The role of the Impacts STG during this process will be to prioritize among various candidate impact events identified by the MQS or science team members, and advocate for the highest-priority event data potentially related to impacts. Priorities may be based on the estimated size and distance to the impact (larger or closer events will be a higher priority), or any unusual aspects of the signal as seen in the continuous data.

\subsection{Orbital Imaging}

Once InSight detects an impact in seismic data and a location estimate is available, images will be requested from one of the currently-orbiting spacecraft around Mars with the goal of pinpointing the exact impact location via visual detection of newly formed crater(s). High resolution images will allow for characterization of the craters' morphology. Exact locations and sizes of the new craters will allow for determination of the ray paths and thus calibrate interior structure models and seismic attenuation. This will drastically reduce the uncertainties in our knowledge of Martian interior structure. Any successful detections will provide a link between the crater size (and thus impact energy) and seismic coupling of impacts, calibrating the seismic efficiency. Each impact site characterized from orbit will additionally reduce the uncertainty on the crater sizes, distances and azimuths estimated by the Marsquake Service. For these reasons, orbital imaging of seismically-detected impact sites will be of high scientific importance.

\subsubsection{Operational Process}

Using the various techniques described in Sect. 6.1, suspected impact events will be distinguished from internal marsquakes in the continuous data from SEIS (Fig. 32). The MQS will provide the science team the estimated location of the detected event, with uncertainties, as well as its type (impact vs. quake). Once an impact event is identified, the Impacts STG will prioritize the downlink of that time period of high-frequency SEIS event data, which is stored on the spacecraft for later retrieval. The initial detection will be accompanied by an estimate from the MQS of the location in azimuth (target uncertainty is $\pm 20^{\circ}$ ), distance (target uncertainty is $\pm 25 \%$ ), and the equivalent tectonic magnitude. These uncertainties are conservative and will improve drastically through the mission using known event locations confirmed in orbital images. Actual uncertainties will also be provided. These are dependent on the number and quality (temporal uncertainty) of the identified phases, the signal-to-noise of the various phases, and the uncertainty in the structural models. The model uncertainty should be reduced as well-located tectonic and impact events are added to the emerging event catalog. The largest and closest events will have smaller uncertainties in terms of area. The location uncertainty could be as small as $10^{\circ}$ in azimuth and $1^{\circ}$ in distance (Panning 


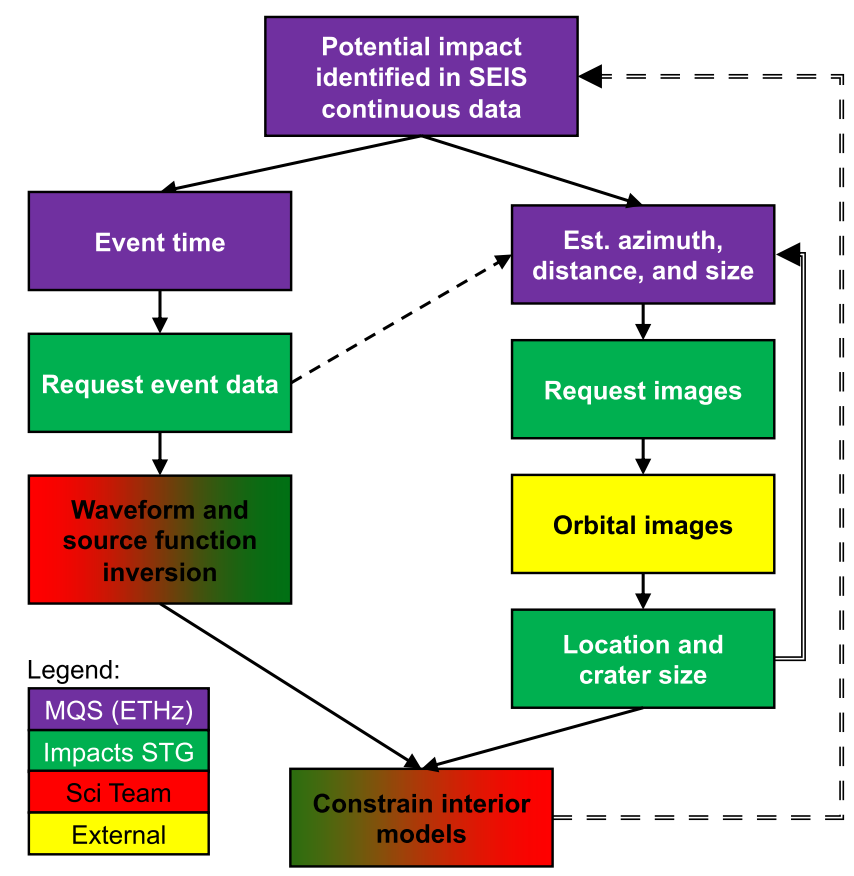

Fig. 32 Schematic of operations planned for impact detection. The color of each step indicates the team responsible. Once a potential impact is detected by the MQS in the continuous SEIS data, two separate flows are initiated. The Impacts STG requests the event data through the weekly event selection process, and also requests orbital images based on the estimated location of the impact. If event data are required to either confirm an impact, or more precisely estimate its location, the image requests will follow acquisition of event data (dashed line). The results of analyzing either the high resolution event data and/or the orbital images will improve estimates of impact locations (double line), and will be used to provide measurements of cratering efficiency and interior properties. Likewise, the constraints on interior models will be fed back into the analysis of new events to improve initial identifications (dashed double line)

et al. 2015) for very large events ( $\sim 1 \mathrm{~km}$ diameter crater). However, impacts this large are exceedingly unlikely to occur within the InSight primary mission: on average, a 1-km crater is formed on Mars approximately once every 10000 years (Hartmann and Daubar 2017). In any case, these uncertainties will be reduced after just a few well-located events are detected and more is learned about the Martian interior.

If the resulting images can provide a crater location and size, these independentlydetermined parameters will be used to improve the algorithms and procedures used by the MQS. When an impact has been confirmed by orbital images, the known position, elevation, and event type (impact) will be entered into subsequent MQS catalogs as fixed values. Further, the magnitude will be recomputed against these location parameters. Most crucially, this fixed and known impact location can be used by the MSS to constrain interior properties of Mars and hence refine candidate models of the Martian structure. These improved models will be used to provide updated seismicity catalogues with improved locations (Sect. 8.1).

Based on the estimated size of the crater, the appropriate imager will be contacted (Table 4). Orbital images will be searched for the extended blast zone around the impact site, which is $\sim 10$ to $\sim 100$ times larger than the craters themselves (Ivanov et al. 2010; Bart and Spinolo 2013); the craters themselves will not be resolved in these initial search images. Very large impacts will be able to be detected in lower-resolution data. The location 
Table 4 Orbiting camera most appropriate for a given impact crater size and distance. Note that individual craters are not expected to be resolved in these data, rather the goal will be to detect the extended blast zone around the impact

\begin{tabular}{lllll}
\hline Imager & Pixel size & Footprint size (approx) & Corresponding crater diameter & Distance range \\
\hline MARCI & $1-10 \mathrm{~km}$ & global map & $>40 \mathrm{~m}$ & Global \\
THEMIS & $18 \mathrm{~m}$ & $20 \mathrm{~km}$ & $\sim 20-40 \mathrm{~m}$ & $\sim 1500-2500 \mathrm{~km}$ \\
CTX & $6 \mathrm{~m}$ & $30 \mathrm{~km} \times 160 \mathrm{~km}$ & $\sim 1-10 \mathrm{~m}$ & $<500 \mathrm{~km}$ \\
CaSSIS $^{1}$ & $5 \mathrm{~m}$ & $8 \mathrm{~km}$ & $\sim 1-10 \mathrm{~m}$ & $<500 \mathrm{~km}$ \\
HiRISE $^{2}$ & $0.25 \mathrm{~m}$ & $1.2 \mathrm{~km} \times 10 \mathrm{~km}$ & All, follow up & All, follow up \\
\hline
\end{tabular}

${ }^{1}$ CaSSIS has limited ability to point off-nadir or target observations

${ }^{2}$ HiRISE will be requested as a follow up in all cases to measure exact crater parameters

uncertainty is a percentage of the estimated distance; thus more distant events will be less well-constrained in areal extent. However, it would be a waste of resources to attempt to search vast areas with many high-resolution images. The number of images needed to cover the location estimate will also depend on the orientation of the region of the location estimate with respect to the spacecraft groundtrack; if the region is elongated along-track, for example, it will be easier to cover with fewer images. The location of the impact will also be taken into account: dusty areas are known to exhibit extended low albedo blast zones around new impacts, aiding their detection in lower-resolution images (Malin et al. 2006; Daubar et al. 2013, 2016). The same size impact in a dust-free area will require higher-resolution images to detect (see Sect. 7.2.2 for more details).

For impacts relatively close to the InSight lander, CTX (6 m/px; Malin et al. 2007) or even HiRISE (25 cm/px; McEwen et al. 2007) images will be requested. Impacts that occur very far from the InSight lander will necessarily be much larger to produce a detectable seismic signal; these may even be detectable in data from Mars Color Imager (MARCI; 1-10 km/px; Bell et al. 2009). MARCI has detected new craters before: $\mathrm{a} \sim 40$ meter crater was discovered that formed between MARCI images on subsequent days (https://www.jpl.nasa.gov/news/news.php?release=2014-162). InSight could also request follow up images from THEMIS (THermal EMission Imaging System on Odyssey; Christensen et al. 2004) for intermediate-sized impacts. Images from the Colour and Stereo Surface Imaging System (CaSSIS) on the Trace Gas Orbiter (TGO) (Thomas et al. 2017) will also be requested; however, that camera's inability to point more than a few degrees off-nadir will limit targeting opportunities.

HiRISE images will be requested for follow-up images, after an impact blast zone is detected in lower-resolution data (with the possible exception of extremely close impacts estimated to be within one HiRISE image width of the InSight lander). Once a new crater is found in lower resolution data, a representative of the Impacts STG will create a target in the public targeting tool HiWish (www.uahirise.org/hiwish/; McEwen et al. 2010), which is available to any member of the scientific or public community. From there, the target will go to the HiRISE team for prioritization and acquisition.

\subsubsection{Image Analysis}

Currently-forming Martian impact craters are relatively small in size (typically $<40 \mathrm{~m}$ in diameter) (Daubar et al. 2013). For the most part, these new craters will only be resolved 


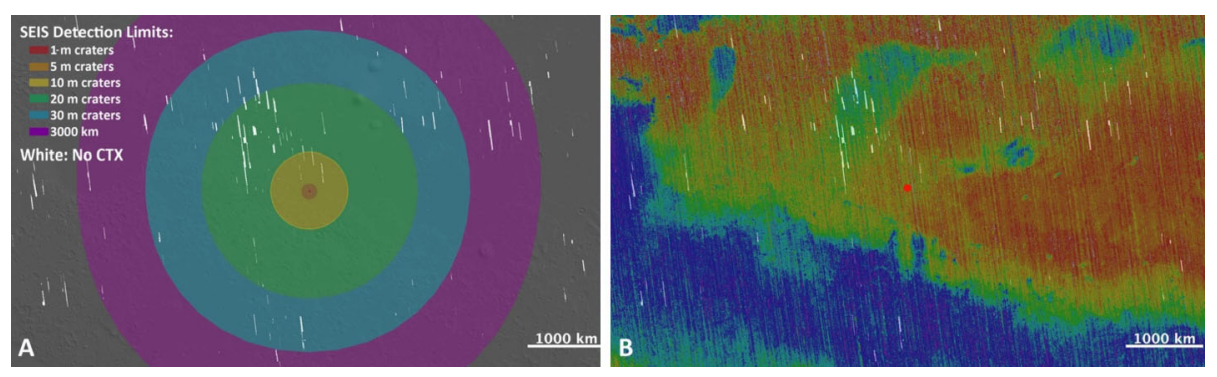

Fig. 33 CTX image coverage (PDS-released images available in JMARS (Christensen et al. 2009) as of January 2018) with (A) detectability of impacts and (B) dust coverage in the InSight landing site area. White areas indicate gaps in CTX coverage at the time of this writing. (A) Colors indicate the distance at which a given size impact can be located, using the relationships estimated in Sect. 6.2. (B) Thermal Emission Spectrometer dust cover index (DCI) (blue = less dust; DCI $<0.96=$ green, yellow, orange, and red) (Ruff and Christensen 2002). Map centered at InSight landing site at $4.5^{\circ} \mathrm{N}, 135.9^{\circ} \mathrm{E}$ (red dot). MOLA shaded relief base courtesy of NASA/JPL/Goddard

in images from the High-Resolution Imaging Science Experiment (HiRISE, $0.25 \mathrm{~m} / \mathrm{pixel}$ ). However, the initial identification of impacts detected by InSight will likely involve detection of the extended "blast zone," a low-albedo area of disturbed dust around the impact, as has been used in the past for new impact detection (Malin et al. 2006; Daubar et al. 2013, 2016). These blast zones enable use of a wider range of imagers for detection of these new impacts and comparison to previous surface conditions. The size of a blast zone relative to the crater size varies widely, ranging from $\sim 10$ to $\sim 100$ times larger (Ivanov et al. 2010; Bart and Spinolo 2013). The InSight landing site is conveniently located in a dusty area (Golombek et al. 2017), the type of surface on which these blast zones form. Dust covers most of area north of InSight, from the northwest to the southeast, but areas to the south and southwest are not dusty (Fig. 33).

Impacts in areas without a surface layer of material with an albedo contrast are much more difficult to detect. Witness the strong bias in detected dated impacts towards dusty areas of Mars (Daubar et al. 2013). Having a relatively high resolution "before" image demonstrating the lack of a crater is thus even more important in dust-free areas. For this reason the number and resolution of images requested, and the thoroughness of search required, will differ depending on whether the estimated location based on seismic data is in a dustcovered or dust-free area.

Previously acquired images will be critical for positively identifying a fresh-looking impact site as new since the most recent image. CTX onboard MRO has covered $99 \%$ of the surface of Mars with > $900006 \mathrm{~m} /$ px images (https://mars.nasa.gov/news/prolific-mars-orbitercompletes-50000-orbits/), so there are few gaps where "before" CTX images are not presently available. In support of the landing site selection process, the InSight landing ellipse region has complete CTX and $>90 \%$ HiRISE coverage (Golombek et al. 2017). Farther from InSight, CTX coverage is nearly complete as well: as of the time of this writing, only a few gaps in coverage remain within a $\sim 3000 \mathrm{~km}$ radius of the InSight landing ellipse (particularly to the north and northwest) (Fig. 33). However, some of the acquired images are poor quality due to dust or haze in the atmosphere. Additional orbital image data will be used to fill those gaps due to missing or poor-quality images. These include data from the THEMIS visible and infrared imaging systems, HiRISE, Mars Orbiter Camera (MOC) (Malin et al. 2010), and the High Resolution Stereo Camera (HRSC) (Neukum and Jaumann 2004; Jaumann et al. 2007; 
Gwinner et al. 2016). As these images are of various ages, the most recent images will be the most valuable.

Remote sensing data for the InSight landing site in western Elysium Planitia suggests it is moderately dusty (Golombek et al. 2017). The relatively high albedo of the InSight landing sites (0.24) argues for a thin coating of dust similar to the dusty portions of the Gusev cratered plains, which have an albedo of 0.26 (Golombek et al. 2005). The TES dust cover index (DCI) (Fig. 33), which includes a more explicit measure of the presence of a thin dust layer (Ruff and Christensen 2002), of the InSight landing site is similar to the VL2 landing site and only slightly dustier than VL1 and Spirit. This value (DCI $=0.94)$ is consistent with a thin coating of dust. The bulk thermal inertia limits the dust layer to less than 1-2 mm thick, and it is more likely a very thin but optically thick veneer of fine grained ( $<$ few micrometers) dust (Golombek et al. 2017). Impacts detected in before and after visible images are preferentially found in areas with DCI $<0.96$ (Daubar et al. 2013). Maps show that most of the surface within $3000 \mathrm{~km}$ of the landing site have DCI values $<0.96$ (fairly dusty) and a relatively high albedo of $>0.2$ (green, yellow, orange and red in Fig. 33B). Thus new impacts in these areas should be detectable in visible images from orbit because they should form a darkened blast zone around the impact site, based on past experiences with new dated impacts on these types of surfaces (Daubar et al. 2013, 2016). Areas $\sim 1000 \mathrm{~km}$ south of the landing site in (blue in Fig. 33B) have a higher dust cover index and lower albedo, both of which imply less dust coverage. This will potentially make orbital detection of new impacts more difficult here.

Orbital images will be manually searched for new impacts by the Impacts Science Theme Group. In dusty areas, fresh impacts are easily recognizable from the low-albedo "blast zone" (Fig. 13). Thus in dusty areas, this search will be fairly straightforward as long as previous images are available, as discussed above. In non-dusty areas, the search will need to be more intense. In both dusty and dust-free areas, if prior images of sufficient quality and resolution are not available, a fresh-appearing impact site found in the area will have a high likelihood of being associated with the event.

\subsubsection{Automated Image Search}

As a supplement to manual searching, and to assist in difficult searches, software is being developed to perform automated image searching. This search will use the Mars Impact Detection Algorithms (MIDA) software developed at Centre National d'Etudes Spatiales (CNES), USGS Integrated Software for Imagers and Spectrometers (ISIS) (e.g., Becker et al. 2013), and in-house image processing that integrates MIDA, ISIS, a geoserver, and a front-end interface. New images of the impact event area will be automatically compared to pre-existing base maps consisting of previous images at global and regional scales. Image information will come from HRSC mosaics ( $20 \mathrm{~m} /$ pixel) at global scale, CTX mosaics $(6 \mathrm{~m} /$ pixel $)$ up to $20^{\circ}(\sim 1000 \mathrm{~km})$ from the lander site, and all available observations that may be available from CaSSIS/TGO (6 m/pixel) and HiRISE $(25 \mathrm{~cm} /$ pixel $)$ inside a circle $5^{\circ}(\sim 300 \mathrm{~km})$ around the lander site. These basemaps are undergoing pre-processing and will be ready for the beginning of the landed mission in November 2018.

The MIDA software uses these basemaps as the basis of comparison for change detection. To produce these, raw CTX images are radiometrically corrected to adjust for mean values of central detectors that are higher than those on the edges of the swath. Each image is orthorectified, sampled at exactly the same pixel size $(5 \mathrm{~m})$, and given an equirectangular projection. Images are then georeferenced to the $100 \mathrm{~m}$ Mars Odyssey THEMIS global mosaic (Edwards et al. 2011) and mosaicked. Algorithms have been built to detect new 
impacts relative to these basemaps, despite changing sun illumination. It is fairly easy for a human to detect impacts in dusty areas, so the challenge for this software is to detect impacts in non-dusty areas. Machine learning approaches are under study to enhance the detection rates while reducing the number of false positives. For more details on this software, see May (2018, submitted).

The automated image search workflow pipeline will be triggered when new image data are available, associated with a MQS event alert of a candidate impact. As we intend to continuously update the basemaps as new orbital observations become available, the MIDA software will also be able to detect new impact craters and/or surface signature changes, even outside the official framework of MQS seismic alerts. The workflow can also be triggered on request by team members.

\subsection{Meteor Imaging}

Meteoroids come in all sizes, including those small enough to ablate completely in the thin Martian atmosphere. These may not be large enough to create craters and seismic signals, but InSight's cameras could still detect the passage of those meteors across the night sky. This would be a direct empirical measurement of the micrometeoroid flux at Mars, which would constrain models of the distribution of small particles in the solar system as a function of distance from the Sun, contributing to constraints on models all sizes of interplanetary bodies.

Night time meteor imaging was first attempted by the MER Rovers, with an initial report of a meteor detection (Selsis et al. 2005). Unfortunately, this was later found consistent with the morphology and size distribution of cosmic rays (Domokos et al. 2007), thus resulting only in an upper limit of the meteoroid flux at Mars. InSight represents another opportunity to pursue this scientific goal at the surface of Mars, and the improved camera sensitivity over those used on MER makes this a promising pursuit.

Predictions of Martian meteor showers bright enough for possible detection by the InSight mission were performed following Vaubaillon et al. (2005) and Vaubaillon (2017). The results are shown in Table 5. The best opportunities result from comets 2004 TG10, 49P, C/1854 L1, and 2002 EV11. However, the first two are long period comets, causing the stream to spread over huge distances, and therefore reducing the meteoroid spatial density.

InSight has two cameras that would be available to image meteors (Maki et al. 2018, this issue). The Instrument Deployment Camera (IDC) and Instrument Context Camera (ICC) on the Insight lander are both flight spare units from the Mars Science Laboratory (MSL) engineering camera development program (Maki et al. 2012), which are copies of the Mars Exploration Rover (MER) engineering cameras (Maki et al. 2003). The IDC is a flight spare MSL Navcam, and the ICC is a flight spare Hazcam. The InSight project has replaced the MSL monochrome detectors with Bayer color filter array (CFA) detectors, removed the neutral density filters, and replaced the visible cutoff filters with IR cutoff filters. The color upgrade has resulted in two main differences relative to the MER/MSL cameras: (1) red, green, and blue bandpasses centered at wavelengths of approximately 450, 550, and $620 \mathrm{~nm}$, respectively, and (2) a factor of five increase in responsivity. This puts the InSight cameras on par with the MER Pancam L1 filter, the most sensitive of the Pancam filters. Other than the color upgrade, the cameras are essentially identical to the MER/MSL versions. For more information on the InSight cameras, see Maki et al. (2018, this issue).

Domokos et al. (2007) found that the MER Pancam L1 (broadband visible) filter could be used to detect meteors to a limiting magnitude of 0.5 to 1.6 , corresponding to meteors 
Table 5 Prediction of meteor showers at Mars. $d$ : Closest distance in astronomical units (AU) between the center of the meteoroid stream and the planet's path. Date: Date of shower (Earth UTC), ZHR: Level of intensity of the shower, i.e. number of meteors a human would witness with the naked eye each hour, under perfect conditions. Conf_index: confidence index as defined in Vaubaillon (2017): a leading "G” for Global indicates that the whole stream is taken into account; $Y$ for Year indicates all predictions are for specific years indicated; following $\mathrm{O}$ for Observations, the number of observations of the body is compared to the number of simulated returns; and finally "CUX.XX" provides information regarding the close encounters the parent body has encountered before it was observed: $\mathrm{X} . \mathrm{XX}=0.00$ indicates that the orbit is fairly well known, and the higher the number X.XX, the higher the uncertainty regarding its past orbit

\begin{tabular}{lrlrl}
\hline Parent & \multicolumn{1}{l}{$d(\mathrm{AU})$} & \multicolumn{1}{l}{ Date } & ZHR & Conf_index \\
\hline 2004 TG10 & -0.01976 & $2018-12-13 \mathrm{~T} 02: 14$ & 111 & GYO0/4CU0.10 \\
4D/Biela & 0.01717 & $2018-11-24 \mathrm{~T} 20: 10$ & 2 & GYO3/38CU0.00 \\
LONEOS-2001R1 & 0.02453 & $2018-12-24 \mathrm{~T} 18: 55$ & 1 & GYO0/28CU0.00 \\
252P/Linear & -0.01940 & $2019-11-16 \mathrm{~T} 08: 35$ & 5 & GYO0/49CU22.58 \\
4D/Biela & 0.00074 & $2019-12-11 \mathrm{~T} 21: 11$ & 3 & GYO3/38CU0.00 \\
49P & 0.00844 & $2019-06-11 T 13: 11$ & 112 & GYO6/6CU0.00 \\
2005 ED318 & 0.02483 & $2019-07-24 T 16: 33$ & 1 & GYO1/21CU0.00 \\
C/1854 L1 & 0.00655 & $2019-09-26 T 22: 29$ & 41 & GYO0/9CU0.00 \\
2002 EV11 & 0.00122 & $2019-11-01 T 23: 12$ & 90 & GYO1/21CU0.00 \\
\hline
\end{tabular}

of $0.1-0.2 \mathrm{~g}$. For that range, they predict $1.4 \times 10^{-5}$ to $5.7 \times 10^{-5}$ meteoroids $\mathrm{km}^{-2} \mathrm{~h}^{-1}$ for a limiting magnitude up to 1.61 and estimate an upper limit value of $<5.4 \times 10^{-6}$ meteoroids $\mathrm{km}^{-2} \mathrm{~h}^{-1}$ for a limiting magnitude up to -4.01 . However, because they could not determine that all streaks were cosmic rays with their methodology, Domokos et al. (2007) caution that the real upper limit may be a few times higher. The InSight cameras are roughly as sensitive as the Pancam L1 filter, and should be sensitive to slightly smaller meteors due to the larger IFOV (at the same angular speed a meteor spends more time within a single IDC or ICC pixel). Due to the larger FOV (FOV of $45^{\circ} \times 45^{\circ}$ ), an IDC image will cover $\sim 8$ times more sky compared to Pancam; aimed at the same elevation (typically $\sim 38^{\circ}$ in the MER meteor searches) the IDC could reproduce the MER results with a total exposure time of about 20 minutes (possible in 4 images). Although the wide field of view $\left(124^{\circ} \times 124^{\circ}\right)$ of the ICC camera offers a larger view of the sky above the horizon, it is fixed mounted to the lander, nominally pointing to the south and only includes low elevations due to aiming for workspace context. The arm-mounted IDC offers the possibility of aiming based on predicted meteor radiants, as well as aiming at elevations with less extinction in dusty times.

Cosmic ray hits are an important source of confusion for meteoroid detection imaging campaigns (e.g., Domokos et al. 2007). We will attempt to identify cosmic rays by exploiting the fact that cosmic rays have no optical point spread function (PSF) as they deposit their energy directly on the detector (effectively bypassing the camera optics), while meteor trails are imaged through the lens system and thus have an optical PSF. We note that, instead of discriminating against cosmic rays via their PSFs, Domokos et al. (2007) relied on pairs of images using two filters of very different sensitivity, and found no paired detections and statistically equivalent distributions of streaks between the two images; they could not specifically rule out faint streaks in the sensitive L1 images if the streaks would not have been detectable in the paired image. Another method to rule out cosmic rays might be to perform simultaneous observations with two separate cameras, such as InSight together 
with MER or MSL. However, such a joint campaign would take significant multi-mission resources.

The Impacts Science Theme Group intends to submit Science Activity Requests to first characterize the meteoroid background and then concentrate imaging campaigns on times when the meteoroid flux is expected to be highest (see Table 5). We will use groups of long exposures, with the exposure length chosen to optimize the detectability of potential meteoroids in light of dark current, read noise, system sensitivity, and cosmic ray flux. Based on the camera sensitivity compared to Pancam, we anticipate that a notional sequence that obtains 20 minutes of integration time over 4-7 images would typically see 1-3 background meteors and require 16-28 Mb of downlink. It is not yet certain whether enough power and data volume will be available for such an imaging campaign to be feasible.

\section{Impact Characterization and Analysis Plans}

\subsection{Validating Interior Structure Models}

In the framework of the InSight mission, impacts will be located by one of several orbiting cameras, which will provide a known location. This will enable the direct inversion of all differential travel times with respect to $\mathrm{P}$ arrival times. If we have epicentral distance and origin time and are able to identify body wave phase arrival times, we have enough information to perform body wave travel times inversion for one dimensional crust and mantle velocity structure along the ray path, using very minimal a priori information. The known location of an impact will enable this analysis, compared to marsquakes that will have much less well-constrained locations.

To test how well an inversion can resolve structure using a limited dataset of only a few impact events, we first invert for the P-wave velocity profile of the Moon using the travel times from artificial impacts acquired by the Apollo 12 station. The artificial events were generated by the impact of the Lunar Modules and the upper stage S-IVB of the Saturn $\mathrm{V}$ rockets with the lunar surface, and most of these impacts correspond to relatively short epicentral distances $(\Delta<300 \mathrm{~km})$. Our study uses 6 artificial impacts for which dates, locations and arrival times can be found in Table 1 of Lognonné et al. (2003). For each ray path, the first $\mathrm{P}$ wave arrival is considered. The reading error attributed to the arrival time estimates is $1 \mathrm{~s}$. Second, to characterize what we could learn about Mars interior structure with only one station, we performed several inversions using a synthetic Martian seismic model, and impacts occurring at different epicentral distances. The Martian model is derived from the Dreibus-Wänke mineralogy profile (Dreibus and Wänke 1985) using the 'hot' endmember temperature profile of Plesa et al. (2016).

The inverse problem consists in a Markov chain Monte Carlo approach, which forms the basis for most of the planned modeling of the Mars Structure Service (MSS) (Panning et al. 2017). This technique allows us to investigate a large range of possible models and provides a quantitative measure of the models' uncertainty and non-uniqueness. The algorithm that we use is explained in Drilleau et al. (2013) and Panning et al. (2015, 2017). The reader is referred to these papers for further details on the practical implementation of the method. The parameterization is done with Bézier points (Bézier 1966, 1967), which are interpolated with $C^{1}$ Bézier curves. The advantages of such a parameterization are that it relies on a small number of parameters that do not need to be regularly spaced in depth, and it can be used to describe both gradients and sharp interfaces. The forward problem consists in a basic ray tracing algorithm (e.g. Shearer 2009). The priors on the parameters are uniformly distributed 
Fig. 34 Inversion results using travel times from artificial impacts on the Moon recorded at Apollo 12 station. Red and blue colors show high and low probability density function (PDF), respectively. The black line is the median profile of the $v_{p}$ distribution as a function of depth, and the black dashed lines represent the interval between $\pm 1 \sigma$ standard deviation. Previously published Moon internal structure models of Garcia et al. (2011), Khan et al. (2014) and Matsumoto et al. (2015) are shown for comparison

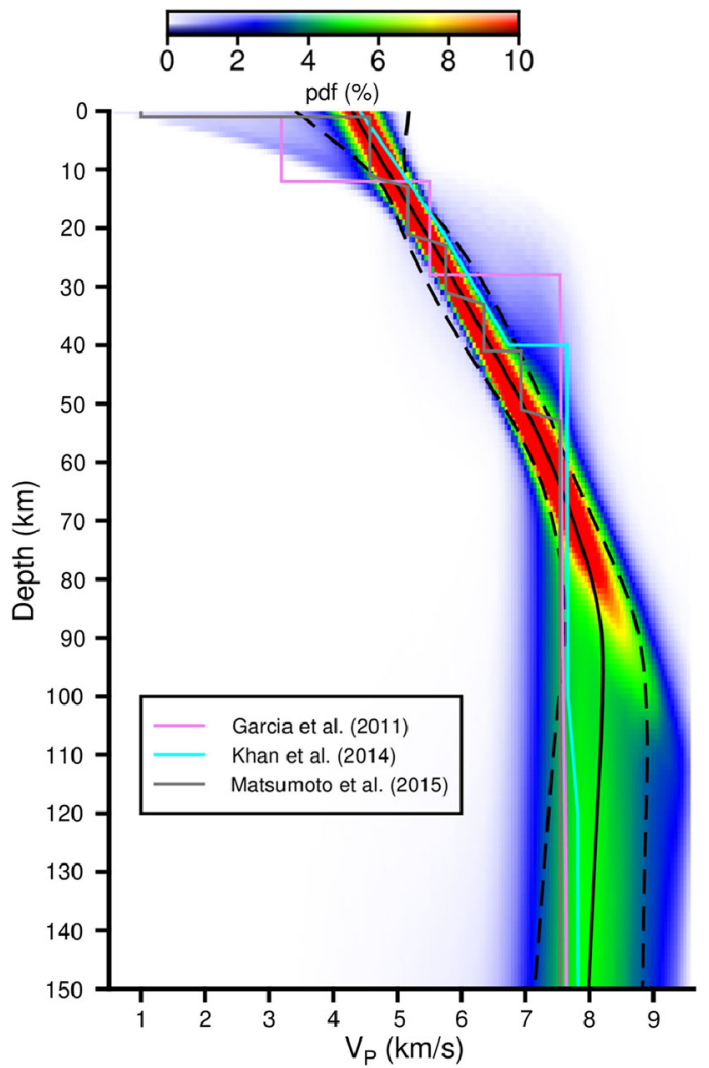

over wide domains. We chose to invoke as few prior constraints as possible to gauge which particular feature is most probable.

The results of the Apollo data inversion are shown in Fig. 34. The plot is a probability density function (PDF) of the accepted models. The $v_{p}$ profile is well defined down to $150 \mathrm{~km}$ depth but not deeper, due to the short epicentral distances where the artificial impacts occurred. The maximum of the PDF shows a $v_{p}$ gradient down to $80 \mathrm{~km}$ depth. Below this depth the profile has a constant value of $\sim 8.1 \mathrm{~km} / \mathrm{s}$. The change in slope could be interpreted as the base of the crust. However, this interpretation must be taken with care because here the depth of an interface is not strictly a model parameter but a useful feature that can be picked in any sampled model. Within the $80-100 \mathrm{~km}$ depth interval, we observe a trade-off between the depth of the slope change and the $v_{p}$ value. This trade-off means, unsurprisingly, that the data fit equally well when the crust-mantle boundary is deeper and $v_{p}$ is higher, or vice-versa. Note that the secondary arrivals, which are very sensitive to sharp interfaces, were picked with very large uncertainties on Apollo data. This was due to the intense scattering in the low-velocity, high-Q upper crust (Dainty et al. 1974) which led to a prolonged, incoherent signal after the initial P arrival. Without the use of such phases, we can only constrain a smooth averaged profile. For comparison, previously published Moon internal structure models of Garcia et al. (2011), Khan et al. (2014) and Matsumoto et al. (2015) are represented in Fig. 34. These three models are made with a layered parameterization. With the exception of the two crustal interfaces of Garcia et al. (2011)'s model and the crust-mantle boundary of Khan et al. (2014)'s model, the three profiles matches well with 


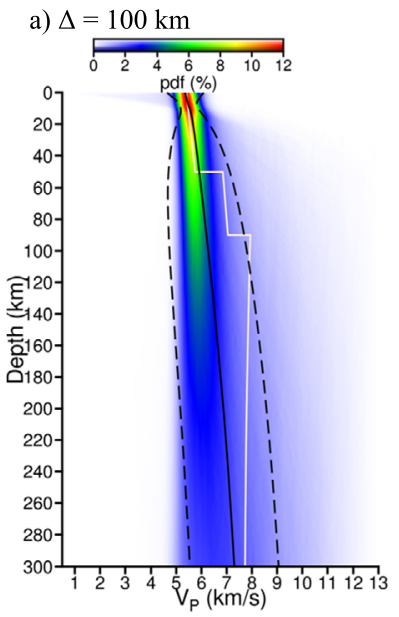

d) $\Delta=100$ and $500 \mathrm{~km}$

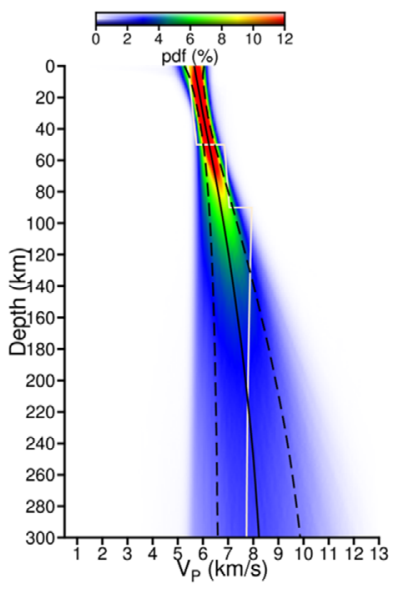

b) $\Delta=500 \mathrm{~km}$

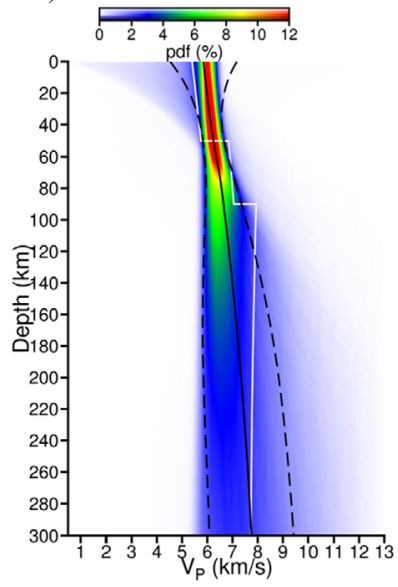

e) $\Delta=100,500$ and $2000 \mathrm{~km}$

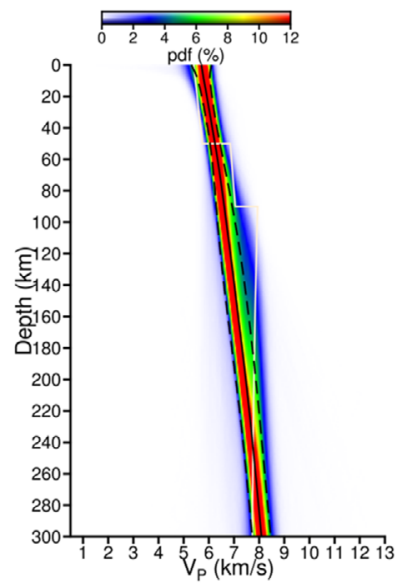

c) $\Delta=2000 \mathrm{~km}$

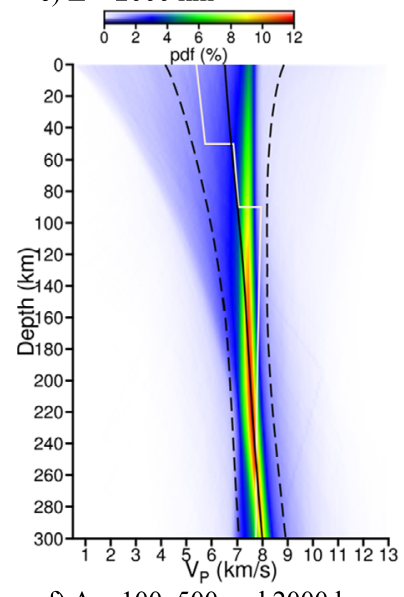

f) $\Delta=100,500$ and $2000 \mathrm{~km}$

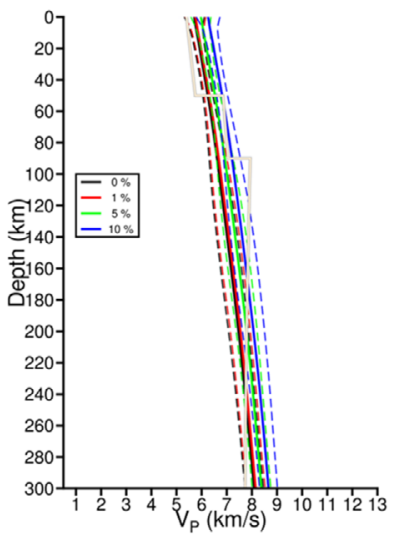

Fig. 35 Results of $v_{p}$ probabilistic inversions using travel times computed for a Martian synthetic model. (a), (b) and (c) show the results performed using only one travel time generated by a single impact, for an epicentral distance of $\Delta=100 \mathrm{~km}, \Delta=500 \mathrm{~km}$ and $\Delta=2000 \mathrm{~km}$, respectively. (d) and (e) show the distributions obtained using 2 impacts at $\Delta=100$ and $500 \mathrm{~km}$, and 3 impacts at $\Delta=100,500$ and $2000 \mathrm{~km}$, respectively. Red and blue colors show high and low probability density functions (PDF), respectively. The black line is the median profile of the $v_{p}$ distribution as a function of depth, and the black dashed lines represent the interval between $\pm 1 \sigma$ standard deviation. The white line is the synthetic model that was input. (f) shows the median and the $\pm 1 \sigma$ standard deviation of the $v_{p}$ distribution when the error on $\Delta$ is equal to $0,1,5$ and $10 \%$

our recovered $v_{p}$ distribution within the $1 \sigma$ uncertainty. Note that between 20 and $50 \mathrm{~km}$ depth, several models show a discontinuity, as shown by the extension of the lower probability blue region of the PDF to higher velocities in this depth range. They are not the most probable models, but they are also able to explain the data within their uncertainty bounds.

The results of the inversion of synthetic $\mathrm{P}$ waves travel times to retrieve Mars interior structure are presented in Fig. 35. Once the InSight lander is operational on Mars, the strategy will be to iteratively improve the interior model as more data becomes available. Con- 
sidering the case at the beginning of the mission, we first show a pessimistic scenario where we investigate what could be retrieved using a single impact event, located at $\Delta=100 \mathrm{~km}$, $500 \mathrm{~km}$ and $2000 \mathrm{~km}$ (Figs. 35a, 35b and 35c). The reading errors are considered to be $1 \mathrm{~s}$, $2.5 \mathrm{~s}$ and $5 \mathrm{~s}$, respectively. In the three cases, the PDFs are the highest and the $1 \sigma$ uncertainties are the lowest at the depths of the turning point of the ray paths. These depths are approximately $5 \mathrm{~km}, 55 \mathrm{~km}$ and $200 \mathrm{~km}$ for $\Delta=100 \mathrm{~km}, 500 \mathrm{~km}$ and $2000 \mathrm{~km}$, respectively. The mode of the distributions and the medians (black lines in Fig. 35) match the input model (white lines in Fig. 35) well at these depths. We also consider a more optimistic scenario likely later in the mission, where we record several impact events located at different epicentral distances. This produces a dataset sensitive to the structure at different depths. Figures $35 \mathrm{~d}$ and $35 \mathrm{e}$ show the inversion results for two impact events located at $\Delta=100 \mathrm{~km}$ and $500 \mathrm{~km}$, and three impacts events located at $\Delta=100 \mathrm{~km}, 500 \mathrm{~km}$ and $2000 \mathrm{~km}$, respectively. In Fig. 35d, we observe that the combination of the two events at $\Delta=100 \mathrm{~km}$ and $500 \mathrm{~km}$ gives a better estimation of the $v_{p}$ profile from the surface down to $35 \mathrm{~km}$ depth, compared to the inversion of the $\Delta=500 \mathrm{~km}$ event alone. With this combination, the model is retrieved down to $80 \mathrm{~km}$ depth. Below this depth, the PDF is broader due to the lack of sensitivity of the data. If a third impact with a larger epicentral distance is added (Fig. 35e), the PDF is tightly constrained down to $300 \mathrm{~km}$ depth. As for the Moon (Fig. 34), the median profile we obtained is smooth compared to the input model, because of the lack of secondary arrivals. However, the PDF is broadened between 80 and $140 \mathrm{~km}$ depth, which indicates a potential change in slope. The good agreement between synthetic and tested data shows here a clear potential to resolve a first order velocity structure of the Martian crust and mantle, using $\mathrm{P}$ wave arrival times of impacts at known locations.

We also investigated to what extent the error on the location would affect the inversion's result. As an example, Fig. $35 \mathrm{f}$ shows the median $v_{p}$ profile and the $1 \sigma$ uncertainties, considering an error of $0 \%,+1 \%,+5 \%$, and $+10 \%$ on the locations of the three impacts. To compensate for the larger epicentral distances, the $v_{p}$ values are higher than in the case where the true epicentral distance is used (black lines). Errors of $+1 \%,+5 \%$, and $+10 \%$ on the locations lead to a $v_{p}$ increase between $0.050-0.074 \mathrm{~km} / \mathrm{s}, 0.27-0.33 \mathrm{~km} / \mathrm{s}$, and $0.55-$ $0.60 \mathrm{~km} / \mathrm{s}$, respectively. Consequently, neglecting the complexities of the three-dimensional structure, we consider that the Level 1 requirement, which is to determine the seismic velocities in the upper $600 \mathrm{~km}$ of the mantle to within $\pm 0.5 \mathrm{~km} / \mathrm{s}$, is met when the error on the epicentral distance is less than $\sim 10 \%$. Low location errors such as these will easily be achievable with impacts that are successfully imaged from orbit.

Another benefit of superficial events such as impacts is that inversions such as this can be used to constrain the crustal thickness at the impact site. In seismic investigations of crustal thickness such as Chenet et al. (2006), the best-constrained location will be the crustal thickness below the seismic station, in this case at the InSight landing site. Because the seismic signals from craters also penetrate through the crust at the impact site, the data can also be used to constrain the crustal thickness there. This will yield additional constraints for lateral variation of the crustal thickness.

\subsection{Measuring Impact-Seismic Efficiency}

Impact-seismic coupling is one of the key aspects in understanding impacts as a seismic source. The seismic efficiency $k$, is not well constrained, with values in the literature ranging from $10^{-6}$ to $10^{-2}$ (see Sect. 3.3 for discussion). Given that no artificial impact is expected during the duration of the InSight mission, we will not be able to calibrate seismic efficiency directly in the way Apollo boosters were used (e.g. Latham et al. 1970a). On the other hand, 
we will be searching for craters associated with seismic events to obtain image data for each impact. This will give us a relationship between crater sizes and seismic energy. The relationship between crater size and impact energy is relatively well known (e.g. Holsapple 1993; Sect. 4.4), and thus we will be able to indirectly evaluate the seismic efficiency.

To precisely evaluate seismic efficiency, sufficient knowledge of the attenuation of Mars is needed. Attenuation is expressed by a quality factor Q. The Q value of Mars will be evaluated through spectral analyses of seismic signals as an activity of the Mars Structure Service (Panning et al. 2017). We will be referring to their model for the correction.

\subsection{Measuring Impactor Size Frequency Distribution}

If sufficient impacts can be detected seismically and imaged in high resolution to resolve their diameters, a measurement of the current impact rate can be made. The impact flux (number of craters of a given size per area, per time) will need to be corrected for the distance at which any given crater diameter is detectable to SEIS. Estimates of these detection limits are discussed in Sect. 6, but will need to be updated with the real performance of the seismometer on the ground at Mars. For example, noise levels at the time of writing can be estimated, but these will not be known with certainty until operation of the seismometer on the surface of Mars. Noise levels will most likely vary with time of day, being lower at night when thermal noise is lower (Murdoch et al. 2017). Another potential observational bias is reduced detections of clustered impacts (Sect. 4.5.1), which comprise half the known impact events at Mars currently (Daubar et al. 2013). These biases will need to be taken into account in the ultimate detection rate calculation. This measurement of the impact flux will be independent of previous measurements that were based on orbital images.

\subsection{Morphologic Study of New Craters}

Images of new craters detected seismically will be used to accurately determine the impact location in longitude and latitude, then converted to offset and azimuth with respect to the location of the SEIS instrument. Once the exact location of the new crater is identified, requests for stereo data will be sent to HiRISE on MRO and CaSSIS on TGO. If stereo images can be obtained, a digital topographic model (DTM) will be created over the area of interest. This can be accomplished using several photogrammetric applications including SOCET SET (Kirk et al. 2003) and Ames Stereo Pipeline (Shean et al. 2016). An estimation of the DTM uncertainties will be performed, similarly to error analysis done for terrestrial data (e.g., Lucas et al. 2015).

If the crater(s) are large enough to be resolved in the data, high resolution images and DTMs will permit several analyses. Images alone will yield a measurement of the crater diameter. Three-dimensional analysis of DTMs will provide the depth, diameter, and excavated crater volume. (Rim height is unlikely to be resolvable, if it is even significant, for these craters.) If DTMs are unavailable or cannot resolve the craters, shadow length measurements can be done to measure the crater depth with less precision (e.g. Daubar et al. 2014). Ejected material and blast zones can be characterized in visible images (spatial extension, directivity) (e.g. Daubar et al. 2016). However, directional blast zones indicating the direction of impact are rare (Daubar et al. 2018). The ejecta is unlikely to be resolved at this scale, thus volume measurements of ejecta will not be likely. If present as a cluster, the geospatial characteristics of the cluster can be studied to reveal impact direction and angle (Daubar et al. 2016, 2018). Characterization of the new craters' morphology and ejecta, together with seismic analyses, may eventually allow an evaluation of the impact velocity and 
direction, impact energy, the mass of impactor, and the porosity of the impacted sub-surface. Geological maps of the area bracketing the position of SEIS and the new crater will also be used to assess the geological context (type and age of the terrains, crustal thickness, regolith depth, etc.) of the impact area and the terrains where waves propagated. The exact location of the impact, the impact direction and energy, and estimation of the sub-surface porosity will help interpret the seismogram recorded by SEIS, including amplitude and arrival time, and constrain lithosphere and regolith models for wave propagation (Sect. 8.1).

\section{Conclusions}

Detecting and studying impacts with Insight will be a challenge, but the wealth of information they will provide about Mars make this a worthwhile pursuit. We will use impacts to achieve the mission goal of measuring the current impact rate at Mars, and also to illuminate the interior structure. A known source location, something that tectonic seismic sources will most likely not be able to accomplish, will enable calibration of the models used to interpret all seismic signals, from marsquakes as well as from impacts. Several impact-specific parameters will be constrained with real data, for example, the source time function (Sect. 3.1) and cutoff frequency (Sect. 3.2). The relationship between the cutoff frequency and impact momentum will be assessed using a known crater size that can be connected to impactor momentum. We will also be able to measure the seismic efficiency (Sect. 3.3), using scaling relationships associating the size of the crater to the impact energy. We will then be able to evaluate the accuracy of our preferred value for the Martian seismic efficiency, $5 \times 10^{-4}$.

We have predicted the frequency of impacts (Sect. 6.2) and the seismic response of Mars (Sect. 5.2) based on our observations of terrestrial (Sect. 2.1), lunar (Sect. 2.2), and experimental impacts (Sect. 3.4). However, the true Martian seismic properties such as seismic efficiency, seismic attenuation, and subsurface velocity structure will not be known until we reach Mars, detect an impact seismically, and calibrate our estimates with orbital images. Enough such detections will also achieve one of the scientific goals of the InSight mission, to measure the impact flux at Mars. This independent measurement of the current impact rate will be free of the biases in previous measurements made using orbital images alone, help us to better understand the chronology of Mars, and clarify the impact hazard to future exploration. Based on current estimates of the Martian impact rate, we predict this measurement will be possible within the timeframe of the prime mission (one Mars year) with the detection of $\sim$ a few to several tens of impacts. Similar measurements of the airburst frequency may also be possible to compare to the predictions we present here (Sect. 4.5.2). Detection of impact-induced acoustic waves may be possible as well (Sect. 4.5.3).

The modeling codes to be used in analysis of the seismic signals from impacts have been benchmarked, and we endorse them for use in future work (Sect. 5). We outlined the processes the InSight Impacts Science Theme Group will follow during mission operations to discriminate impacts from marsquakes (Sect. 6.1); follow up on impact seismic detections (Sect. 7.1); request event data and orbital images (Sect. 7.2); search those images for the impact site (Sects. 7.2.2 and 7.2.3); and finally analyze those data (Sect. 8). A plan for possible night-time meteor imaging was also presented (Sect. 7.3); this valuable, but not required, experiment would provide a direct measurement of the small end of the size distribution of the Martian impact flux.

Using data from InSight, these analyses will lead to better understanding of the shallow subsurface structure, physical and seismic properties of the interior, the seismic efficiency and other seismic-impact parameters, and the current impact flux at Mars. 
Acknowledgements We are grateful to Jay Melosh and an unnamed reviewer for thoughtful and helpful comments. We appreciate the hard work of the engineering and operations teams who are making the InSight mission possible. Elizabeth Barrett provided valuable input. Thank you to Matthew Siegler for helping to address a reviewer comment. A portion of this research was carried out at the Jet Propulsion Laboratory, California Institute of Technology, under a contract with the National Aeronautics and Space Administration. French co-authors thank the support of the French Space Agency CNES as well as ANR SIMARS. IPGP coauthors (IPGP contribution number 3988) also received support from the UnivEarth Labex at Sorbonne Paris Cité (ANR-10-LABX-0023 and ANR-11-IDEX-0005-02). N. Teanby and J. Wookey are funded by the UK Space Agency. Swiss co-authors recognize the support of the (1) Swiss National Science Foundation and French Agence Nationale de la Recherche (SNF-ANR project 157133 "Seismology on Mars") and (2) Swiss State Secretariat for Education, Research and Innovation (SEFRI project "MarsQuake Service-Preparatory Phase"). We gratefully acknowledge the developers of the iSALE hydrocode. A portion of this work was performed using HPC resources of CINES (Centre Informatique National de l'Enseignement Supérieur) under the allocation A0030407341 made by GENCI (Grand Equipement National de Calcul Intensif). KM research is fully supported by the Australian Government (project numbers DE180100584 and DP180100661). This is InSight Contribution Number 47.

\section{References}

K. Aki, P.G. Richards, Quantitative Seismology, 2nd edn. (University Science Books, Herndon, 2002), ISBN 0-935702-96-2, 704 pp.

D.L. Anderson, F.K. Duennebier, G.V. Latham, M.F. Toksöz, R.L. Kovach, T.C. Knight, G. Sutton, The Viking seismic experiment. Science 194(4271), 1318-1321 (1976). https://doi.org/10.1126/science.194. 4271.1318

N. Artemieva, E. Pierazzo, The Canyon Diablo impact event: projectile motion through the atmosphere. Meteorit. Planet. Sci. 44(1), 25-42 (2009)

B. Baldwin, Y. Sheaffer, Ablation and breakup of large meteoroids during atmospheric entry. J. Geophys. Res. 76(19), 4653-4668 (1971)

W.B. Banerdt et al., The InSight mission. Space Sci. Rev. (2018, this issue)

W.B. Banerdt, S. Smrekar, P. Lognonné, T. Spohn, S.W. Asmar, D. Banfield, L. Boschi, U. Christensen, V. Dehant, W. Folkner, D. Giardini, W. Goetze, M. Golombek, M. Grott, T. Hudson, C. Johnson, G. Kargl, N. Kobayashi, J. Maki, D. Mimoun, A. Mocquet, P. Morgan, M. Panning, W.T. Pike, J. Tromp, T. van Zoest, R. Weber, M.A. Wieczorek, R. Garcia, K. Hurst, InSight: a discovery mission to explore the interior of Mars, in Lunar and Planetary Science Conference, Lunar and Planetary Inst. Technical Report 44 (2013), p. 1915

W.B. Banerdt, S.E. Smrekar, T. Hoffman, S. Spath, P. Lognonné, T. Spohn, H. Stone, J. Willis, J. Feldman, R. De Paula, R. Turner, S. Asmar, D. Banfield, U. Christensen, J. Clinton, V. Dehant, W. Folkner, R. Garcia, D. Giardini, M. Golombek, M. Grott, T. Hudson, C. Johnson, G. Kargl, B. Knapmeyer-Endrun, J. Maki, D. Mimoun, A. Mocquet, P. Morgan, M. Panning, W.T. Pike, C. Russell, N. Teanby, J. Tromp, R. Weber, M. Wieczorek, K. Hurst, E. Barrett (The InSight Team), The InSight mission for 2018, in Lunar \& Planetary Science Conference 48 (2017), abstract 1896

M.E. Banks, I.J. Daubar, N.C. Schmerr, M.P. Golombek, Predicted seismic signatures of recent dated Martian impact events: implications for the InSight lander, in Lunar and Planetary Science Conference 46 (2015), abstract 2679

G.D. Bart, P.L. Spinolo, Formation of airblast features associated with young Martian craters. Abstr. Program - Geol. Soc. Am. 45(7), 200-10 (2013)

H.E. Bass, J.P. Chambers, Absorption of sound in the Martian atmosphere. J. Acoust. Soc. Am. 109, 2371 (2001). https://doi.org/10.1121/1.4744345

K.J. Becker et al., ISIS support for NASA mission instrument ground data processing systems, in Lunar Planet. Sci. XLIV, Houston, Texas (2013), abstract \#2829

J.F. Bell, M.J. Wolff, M.C. Malin, W.M. Calvin, B.A. Cantor, M.A. Caplinger, P.C. Thomas, Mars Reconnaissance Orbiter Mars Color Imager (MARCI): instrument description, calibration, and performance. J. Geophys. Res. 114(E8), E08S92 (2009). https://doi.org/10.1029/2008JE003315

A. Ben-Menahem, Source parameters of the Siberian explosion of June 30, 1908, from analysis and synthesis of seismic signals at four stations. Phys. Earth Planet. Inter. 11(1), 1-35 (1975). https://doi.org/ 10.1016/0031-9201(75)90072-2

P. Bézier, Définition numérique des courbes et surfaces I. Automatisme 11, 625-632 (1966)

P. Bézier, Définition numérique des courbes et surfaces II. Automatisme 12, 17-21 (1967) 
M. Böse, J. Clinton, S. Ceylan, F. Euchner, M. van Driel, A. Khan, D. Giardini, P. Lognonné, W.B. Banerdt, A probabilistic framework for single-station location of seismicity on Earth and Mars. Phys. Earth Planet. Inter. 262, 48-65 (2017). https://doi.org/10.1016/j.pepi.2016.11.003

M. Böse, D. Giardini, S. Stähler, S. Ceylan, J. Clinton, M. van Driel, A. Khan, F. Euchner, P. Lognonné, B. Banerdt Magnitude Scales for Marsquakes (2018, in press)

P. Brown, D.O. ReVelle, E.A. Silber, W.N. Edwards, S. Arrowsmith, L.E. Jackson, G. Tancredi, D. Eaton, Analysis of a crater-forming meteorite impact in Peru. J. Geophys. Res. 113, E09007 (2008)

P.G. Brown, J.D. Assink, L. Astiz, R. Blaauw, M.B. Boslough, J. Borovička, N. Brachet, D. Brown, M. Campbell-Brown, L. Ceranna et al., A 500-kiloton airburst over Chelyabinsk and an enhanced hazard from small impactors. Nature 503, 238-241 (2013)

K.J. Burleigh, H.J. Melosh, L.L. Tornabene, B. Ivanov, A.S. McEwen, I.J. Daubar, Impact airblast triggers dust avalanches on Mars. Icarus 217, 194-201 (2012)

H. Chenet, P. Lognonné, M.A. Wieczorek, H. Mizutani, Lateral variations of lunar crustal thickness from the Apollo seismic data set. Earth Planet. Sci. Lett. 243, 1-14 (2006). https://doi.org/10.1016/j.epsl. 2005.12.017

P.R. Christensen, B.M. Jakosky, H.H. Kieffer, M.C. Malin, H.Y. McSween Jr., K. Nealson, G.L. Mehall, S.H. Silverman, S. Ferry, M. Caplinger, M. Ravine, The Thermal Emission Imaging System (THEMIS) for the Mars 2001 Odyssey Mission. Space Sci. Rev. 110, 85-130 (2004)

P.R. Christensen, E. Engle, S. Anwar, S. Dickenshied, D. Noss, N. Gorelick, M. Weiss-Malik, JMARS-a planetary GIS, in American Geophysical Union Fall Meeting, IN22A-06 (2009)

C.F. Chyba, P.J. Thomas, K.J. Zahnle, The 1908 tunguska explosion: atmospheric disruption of a stony asteroid. Nature 361(6407), 40-44 (1993)

É. Clévédé, P. Lognonné, 85.16 higher order perturbation theory: 3D synthetic seismogram package. Int. Geophys. 81, 1639 (2003). https://doi.org/10.1016/S0074-6142(03)80295-4

Clinton et al., Marsquake Service-building a Martian seismicity catalogue for InSight. Space Sci. Rev. (2018, this issue)

G.S. Collins, H.J. Melosh, R.A. Marcus, Earth impact effects program: a web-based computer program for calculating the regional environmental consequences of a meteoroid impact on Earth. Meteorit. Planet. Sci. 40(6), 817-840 (2005)

A.M. Dainty, M.N. Toksz, K.R. Anderson, P.J. Pines, Y. Nakamura, G. Latham, Seismic scattering and shallow structure of the Moon in Oceanus Procellarum. Moon 9, 11-29 (1974)

I.J. Daubar, A.S. McEwen, S. Byrne, M.R. Kennedy, B. Ivanov, The current Martian cratering rate. Icarus 225, 506-516 (2013). https://doi.org/10.1016/j.icarus.2013.04.009

I.J. Daubar, C. Atwood-Stone, S. Byrne, A.S. McEwen, P.S. Russell, The morphology of small fresh craters on Mars and the Moon. J. Geophys. Res., Planets 119, 2620-2639 (2014). https://doi.org/10.1002/ 2014JE004671

I.J. Daubar, M.P. Golombek, A.S. McEwen, S. Byrne, M.A. Kreslavsky, N.C. Schmerr, M.E. Banks, Measurement of the current Martian cratering size frequency distribution, predictions for and expected improvements from InSight, in Lunar and Planetary Science Conference Abstracts (2015), abstract 2468

I.J. Daubar, C.M. Dundas, S. Byrne, P.E. Geissler, G.D. Bart, A.S. McEwen, M. Chojnacki, Changes in blast zone Albedo patterns around new Martian impact craters. Icarus 267, 86-105 (2016). https://doi.org/ 10.1016/j.icarus.2015.11.032

I.J. Daubar, M.E. Banks, N.C. Schmerr, M.P. Golombek, Recently Formed Crater Clusters on Mars (2018, in press)

D.M. Davis, Meteoroid impacts as seismic sources on. Mars 105, 469-478 (1993)

P. Delage, F. Karakostas, A. Dhemaied, M. Belmokhtar, P. Lognonné, M. Golombek, E. De Laure, K. Hurst, J.-C. Dupla, S. Kedar, Y.J. Cui, B. Banerdt, An investigation of the mechanical properties of some Martian regolith simulants with respect to the surface properties at the InSight mission landing site. Space Sci. Rev. 211, 191-213 (2017). https://doi.org/10.1007/s11214-017-0339-7

A. Domokos, J.F. Bell III., P. Brown, M.T. Lemmon, R. Suggs, J. Vaubaillon, W. Cook, Measurement of the meteoroid flux at Mars. Icarus 191, 141-150 (2007). https://doi.org/10.1016/j.icarus.2007.04.017

G. Dreibus, H. Wänke, Mars: a volatile rich planet. Meteoritics 20, 367-382 (1985)

M. Drilleau, E. Beucler, A. Mocquet, O. Verhoeven, G. Moebs, G. Burgos, J.P. Montagner, P. Vacher, A Bayesian approach to infer radial models of temperature and anisotropy in the transition zone from surface wave dispersion curves. Geophys. J. Int. 195, 1165-1183 (2013)

W.N. Edwards, D.W. Eaton, P.G. Brown, Seismic observations of meteors: coupling theory and observations. Rev. Geophys. 46, 2007RG000253 (2008)

C.S. Edwards, K.J. Nowicki, P.R. Christensen, J. Hill, N. Gorelick, K. Murray, Mosaicking of global planetary image datasets: 1. Techniques and data processing for Thermal Emission Imaging System (THEMIS) multi-spectral data. J. Geophys. Res. 116, E10008 (2011). https://doi.org/10.1029/2010JE003755 
W. Friederich, J. Dalkolmo, Complete synthetic seismograms for a spherically symmetric Earth by a numerical computation of the Green's function in the frequency domain. Geophys. J. Int. 122, 537-550 (1995). https://doi.org/10.1111/j.1365-246X.1995.tb07012.x

J. Gagnepain-Beyneix, P. Lognonné, H. Chenet, D. Lombardi, T. Spohn, A seismic model of the lunar mantle and constraints on temperature and mineralogy. Phys. Earth Planet. Inter. 159(3-4), 140-166 (2006). https://doi.org/10.1016/j.pepi.2006.05.009

R.F. Garcia, J. Gagnepain-Beyneix, S. Chevrot, P. Lognonné, Very preliminary reference Moon model. Phys. Earth Planet. Inter. 188, 96-113 (2011). https://doi.org/10.1016/j.pepi.2011.06.015

R.F. Garcia, Q. Brissaud, L. Rolland, R. Martin, D. Komatitsch, A. Spiga, P. Lognonné, W.B. Banerdt, Finite-difference modeling of acoustic and gravity wave propagation in Mars atmosphere: application to infrasounds emitted by meteor impacts. Space Sci. Rev. 211(1-4), 547-570 (2017). https://doi.org/10.1007/s11214-016-0324-6

J.R. Geller, T. Ohminato, Computation of synthetic seismograms and their partial derivatives for heterogeneous media with arbitrary natural boundary conditions using the direct solution method. Geophys. J. Int. 116(2), 421-446 (1994)

R.J. Geller, N. Takeuchi, A new method for computing highly accurate DSM synthetic seismograms. Geophys. J. Int. 123, 449-470 (1995)

F. Gilbert, A.M. Dziewonski, An application of normal mode theory to the retrieval of structural parameters and source mechanism from seismic spectra. Philos. Trans. R. Soc. A 278, 1280 (1975). https://doi.org/10.1098/rsta.1975.0025

M.P. Golombek, A revision of Mars seismicity from surface faulting, in Lunar Planet. Sci. Conf. XXXIII (2002), abstract 1244

M.P. Golombek, W.B. Banerdt, K.L. Tanaka, D.M. Tralli, A prediction of Mars seismicity from surface faulting. Science 258, 979-981 (1992)

M.P. Golombek et al., Assessment of Mars Exploration Rover landing site predictions. Nature 436, 44-48 (2005). https://doi.org/10.1038/nature03600

M.P. Golombek, A.F.C. Haldemann, R.A. Simpson, R.L. Fergason, N.E. Putzig, R.E. Arvidson, J.F. Bell III., M.T. Mellon, Martian surface properties from joint analysis of orbital, Earth-based, and surface observations, in The Martian Surface: Composition, Mineralogy and Physical Properties, ed. by J.F. Bell III. (Cambridge University Press, Cambridge, 2008), pp. 468-497. Chap. 21

M. Golombek, D. Kipp, N. Warner, I.J. Daubar, R. Fergason, R. Kirk, R. Beyer, A. Huertas, S. Piqueux, N.E. Putzig, B.A. Campbell, G.A. Morgan, C. Charalambous, W.T. Pike, K. Gwinner, F. Calef, D. Kass, M. Mischna, J. Ashley, C. Bloom, N. Wigton, T. Hare, C. Schwartz, H. Gengl, L. Redmond, M. Trautman, J. Sweeney, C. Grima, I.B. Smith, E. Sklyanskiy, M. Lisano, J. Benardini, S. Smrekar, P. Lognonné, W.B. Banerdt, Selection of the InSight landing site. Space Sci. Rev. 211, 5-95 (2017). https://doi.org/10.1007/s11214-016-0321-9

M.P. Golombek et al., Geology and physical properties investigations by the InSight lander. Space Sci. Rev. 214(5), 1-52 (2018). https://doi.org/10.1007/s11214-018-0512-7

T. Gudkova, P. Lognonné, J. Gagnepain-Beyneix, Large impacts detected by the Apollo seismometers: impactor mass and source cutoff frequency estimations. Icarus 211, 1049-1065 (2011)

T.V. Gudkova, P. Lognonné, K. Miljković, J. Gagnepain-Beyneix, Impact cutoff frequency-momentum scaling law inverted from Apollo seismic data. Earth Planet. Sci. Lett. 427, 57-65 (2015). https://doi.org/ 10.1016/j.eps 1.2015 .06 .037

N. Güldemeister, K. Wünnemann, Quantitative analysis of impact-induced seismic signals by numerical modeling. Icarus 296, 15-27 (2017)

K. Gwinner et al., The High Resolution Stereo Camera (HRSC) of Mars Express and its approach to science analysis and mapping for Mars and its satellites. Planet. Space Sci. (2016). https://doi.org/10.1016/j.pss.2016.02.014

W.K. Hartmann, Martian cratering. Icarus 5(1-6), 565-576 (1966). https://doi.org/10.1016/00191035(66)90071-6

W.K. Hartmann, Relative crater production rates on planets. Icarus 31(2), 260-276 (1977)

W.K. Hartmann, Martian cratering 8: isochron refinement and the chronology of Mars. Icarus 174, 294-320 (2005)

W.K. Hartmann, I.J. Daubar, Martian cratering 11. Utilizing decameter scale crater populations to study Martian history. Meteorit. Planet. Sci. 52, 493-510 (2017). https://doi.org/10.1111/maps.12807

W.K. Hartmann, I.J. Daubar, O.P. Popova, E. Joseph, Martian cratering 12. Utilizing primary crater clusters to study crater populations and meteoroid properties. Meteorit. Planet. Sci. (2017, in press). https://doi.org/ 10.1111/maps.13042

N.A. Haskell, Analytic approximation for the elastic radiation from a contained underground explosion. J. Geophys. Res. 72, 2583-2587 (1967). https://doi.org/10.1029/JZ072i010p02583 
M.A.H. Hedlin, B.W. Stump, D.C. Pearson, X. Yang, Identification of mining blasts at mid- to far-regional distances using low frequency seismic signals, in Monitoring the Comprehensive Nuclear-Test-Ban Treaty: Seismic Event Discrimination and Identification. Pageoph Topical Volumes, ed. by W.R. Walter, H.E. Hartse (Birkhäuser, Basel, 2002)

D.C. Helmberger, D.M. Hadley, Seismic source functions and attenuation from local and teleseismic observations of the NTS events Jorum and Handley. Bull. Seismol. Soc. Am. 71(1), 51-67 (1981)

R.B. Herrmann, Computer programs in seismology: an evolving tool for instruction and research. Seismol. Res. Lett. 84, 1081-1088 (2013). https://doi.org/10.1785/0220110096

J.G. Hills, M.P. Goda, The fragmentation of small asteroids in the atmosphere. Astron. J. 105, 1114-1144 (1993)

K.A. Holsapple, The scaling of impact processes in planetary sciences. Annu. Rev. Earth Planet. Sci. 21, 333-373 (1993). https://doi.org/10.1146/annurev.ea.21.050193.002001

K. Holsapple, K.R. Housen, A crater and its ejecta: an interpretation of deep impact. Icarus 187, 345-356 (2007)

K.A. Holsapple, R.M. Schmidt, Point source solutions and coupling parameters in cratering mechanics. J. Geophys. Res. 92, 6350-6376 (1987). https://doi.org/10.1029/JB092iB07p06350

B. Ivanov, Mars/Moon cratering rate ratio estimates. Space Sci. Rev. 96, 87-104 (2001). https://doi.org/ 10.1023/A:1011941121102

B.A. Ivanov, N.A. Artemieva, Numerical modeling of the formation of large impact craters, in Catastrophic events and mass extinctions: Impacts and beyond, ed. by C. Koeberl, K.G. MacLeod. Geological Society of America Special Paper, vol. 356 (2002), pp. 619-630

B. Ivanov, D. Deniem, G. Neukum, Implementation of dynamic strength models into 2d hydrocodes: applications for atmospheric breakup and impact cratering. Int. J. Impact Eng. 20(1), 411-430 (1997)

B.A. Ivanov, H.J. Melosh, A.S. McEwen, New small impact craters in high resolution HiRISE images-III, in Lunar and Planetary Science Conference Abstracts (2010), abstract 2020

R. Jaumann, G. Neukum, T. Behnke, T.C. Duxbury, E. Eichentopf, H. Hoffmann, A. Hoffmeister, U. Köhler, K-D. Matz, T.B. McCord, V. Mertens, J. Obserst, R. Pischel, D. Reiss, E. Ress, T. Roatsch, P. Saiger, F. Scholten, G. Schwartz, K. Stephan, M. Wählisch (the HRSC Co-Investigation Team), The HighResolution Stereo Camera (HRSC) experiment on the Mars Express: instrument aspects and experiment conduct from interplanetary cruise through the nominal mission. Planet. Space Sci. 55, 928-952 (2007)

Y. JeongAhn, R. Malhotra, The current impact flux on Mars and its seasonal variation. Icarus 262, 140-153 (2015)

K. Kawai, N. Takeuchi, R.J. Geller, Geoph. J. Int. 164, 411 (2006)

T. Kawamura, P. Lognonné, Y. Nishikawa, S. Tanaka, Evaluation of deep moonquake source parameters: implication for fault characteristics and thermal state. J. Geophys. Res., Planets 122, 1487-1504 (2017). https://doi.org/10.1002/2016JE005147

S. Kedar, J. Andrade, W. Bruce Banerdt, P. Delage, M.P. Golombek, M. Grott, T. Hudson et al., Analysis of regolith properties using seismic signals generated by InSight's HP3 penetrator. Space Sci. Rev. 211(14), 315-337 (2017). https://doi.org/10.1007/s11214-017-0391-3

A. Khan, K. Mosegaard, An inquiry into the lunar interior: a nonlinear inversion of the Apollo lunar seismic data. J. Geophys. Res. 107(E6), 5036 (2002). https://doi.org/10.1029/2001JE001658

A. Khan, J.A.D. Connolly, A. Pommier, J. Noir, Geophysical evidence for melt in the deep lunar interior and implications for lunar evolution. J. Geophys. Res., Planets 119(10), 2197-2221 (2014). https://doi.org/10.1002/2014JE004661

A. Khan, M. van Driel, M. Böse, D. Giardini, S. Ceylan, J. Yan, J. Clinton, F. Euchner, P. Lognonné, N. Murdoch, D. Mimoun, M. Panning, M. Knapmeyer, W.B. Banerdt, Single-station and single-event marsquake location and inversion for structure using synthetic Martian waveforms. Phys. Earth Planet. Inter. 258, 28-42 (2016). https://doi.org/10.1016/j.pepi.2016.05.017

R.L. Kirk, E. Howington-Kraus, B. Redding, D. Galuszka, T.M. Hare, B.A. Archinal, L.A. Soderblom, J.M. Barrett, High-resolution topomapping of candidate MER landing sites with Mars Orbiter Camera narrow-angle images. J. Geophys. Res. 108(E12), 29 (2003). https://doi.org/10.1029/2003JE00213

M. Knapmeyer, J. Oberst, E. Hauber, M. Wählisch, C. Deuchler, R. Wagner, Working models for spatial distribution and level of Mars' seismicity. J. Geophys. Res. 111, E11006 (2006). https://doi.org/10.1029/2006JE002708

B. Knapmeyer-Endrun, M.P. Golombek, M. Ohrnberger, Rayleigh wave ellipticity modeling and inversion for shallow structure at the proposed InSight landing site in Elysium Planitia, Mars. Space Sci. Rev. 211, 339-382 (2017). https://doi.org/10.1007/s11214-016-0300-1

D. Komatitsch, J.P. Vilotte, The spectral element method: an efficient tool to simulate the seismic response of 2D and 3D geological structures. Bull. Seismol. Soc. Am. 88(2), 368-392 (1998)

L.D. Landau, E.M. Lifshitz, Electrodynamic of Solids (Science, Moscow, 1982) 
G. Latham, M. Ewing, J. Dorman, F. Press, N. Toksoz, G. Sutton, R. Meissner, F. Duennebi, Y. Nakamura, R. Kovach, M. Yates, Seismic data from man-made impacts on the Moon. Science 170, 620-626 (1970a)

G.V. Latham, W.G. McDonald, H.J. Moore, Missile impacts as sources of seismic energy on the Moon. Science 168, 242-245 (1970b)

M. Le Feuvre, M.A. Wieczorek, Nonuniform cratering of the Moon and a revised crater chronology of inner Solar System. Icarus 214, 1-20 (2011)

A. Le Pichon, K. Antier, Y. Cansi, B. Hernandez, E. Minaya, B. Burgoa, D. Drob, L.G. Evers, J. Vaubaillon, Evidence for a meteoritic origin of the September 15, 2007, Carancas crater. Meteorit. Planet. Sci. 43, 1797-1809 (2008)

P. Lognonné et al., SEIS: The Seismic Experiment for Internal Structure of InSight. Space Sci. Rev. (2018, this issue)

P. Lognonné, E. Clévédé, 10 normal modes of the Earth and planets. Int. Geophys. 81, 125 (2002). https:// doi.org/10.1016/S0074-6142(02)80213-3

P. Lognonné, C. Johnson, Planetary seismology, in Treatise on Geophysics, vol. 10 (2007), pp. 69-122. https:// doi.org/10.1016/B978-044452748-6.00154-1

P. Lognonné, C.L. Johnson, Planetary seismology, in Treatise on Geophysics, ed. by G. Schubert 2 nd edn. (Elsevier, Oxford, 2015), pp. 65-120. https://doi.org/10.1016/B978-0-444-53802-4.00167-6. ISBN 9780-444-53803-1

P. Lognonné, T. Kawamura, Impact seismology on terrestrial and giant planets, in Extraterrestrial Seismology, ed. by V. Tong, R. García (Cambridge University Press, Cambridge, 2015), pp. 250-263. https://doi.org/10.1017/CBO9781107300668.021

P. Lognonné, B. Mosser, Planetary seismology. Surv. Geophys. 14(3), 239-302 (1993). https://doi.org/ 10.1007/BF00690946

P. Lognonné, B. Mosser, F.A. Dahlen, Excitation of the Jovian seismic waves by the Shoemaker-Levy 9 cometary impact. Icarus 110, 186-195 (1994). https://doi.org/10.1006/icar.1994.1115

P. Lognonné, J. Gagnepain-Beyneix, H. Chenet, A new seismic model of the Moon: implications for structure, thermal evolution and formation of the Moon. Earth Planet. Sci. Lett. 211, 27-44 (2003)

P. Lognonné, M. Le Feuvre, C.L. Johnson, R.C. Weber, Moon meteoritic seismic hum: steady state prediction. J. Geophys. Res. 114, E12003 (2009)

P. Lognonné, F. Karakostas, L. Rolland, Y. Nishikawa, Modeling of atmospheric-coupled Rayleigh waves on planets with atmosphere: from Earth observation to Mars and Venus perspectives. J. Acoust. Soc. Am. 140(2), 1447-1468 (2016)

R.D. Lorenz, M. Panning, Empirical recurrence rates for ground motion signals on planetary surfaces. Icarus 303, 273-279 (2017). https://doi.org/10.1016/j.icarus.2017.10.008

A. Lucas, C. Narteau, S. Rodriguez, O. Rozier, Y. Callot, A. Garcia, S. Courrech Du Pont, Sediment flux from the morphodynamics of elongating linear dunes. Geology (2015). https://doi.org/10.1130/G37101.1

Maki et al., The Mars Insight Lander Cameras. Space Sci. Rev. (2018, this issue)

J.N. Maki, J.F. Bell, K.E. Herkenhoff, S.W. Squyres, A. Kiely, M. Klimesh, M. Schwochert, T. Litwin, R. Willson, A. Johnson, M. Maimone, E. Baumgartner, A. Collins, M. Wadsworth, S.T. Elliot, A. Dingizian, D. Brown, E.C. Hagerott, L. Scherr, R. Deen, D. Alexander, J. Lorre, The Mars exploration rover engineering cameras. J. Geophys. Res. 108(E12), 8071 (2003). https://doi.org/10.1029/2003JE002077

J. Maki, D. Thiessen, A. Pourangi, P. Kobzeff, T. Litwin, L. Scherr, S. Elliott, A. Dingizian, M. Maimone, The Mars science laboratory engineering cameras. Space Sci. Rev. 170, 77-93 (2012). https://doi.org/10.1007/s11214-012-9882-4

M.C. Malin, K.S. Edgett, L.V. Posiolova, S.M. McColley, E.Z.N. Dobrea, Present-day impact cratering rate and contemporary gully activity on Mars. Science 314, 1573-1577 (2006)

M.C. Malin et al., Context camera investigation on board the Mars reconnaissance orbiter. J. Geophys. Res. 112, E05S04 (2007). https://doi.org/10.1029/2006JE002808

M.C. Malin, K.S. Edgett, B.A. Cantor, M.A. Caplinger, G.E. Danielson, E.H. Jensen, K.D. Supulver, An overview of the 1985-2006 Mars Orbiter Camera science investigation. Mars J. 5, 1-60 (2010). https://doi.org/10.1555/mars.2010.0001

K. Matsumoto, R. Yamada, F. Kikuchi, S. Kamata, Y. Ishihara, T. Iwata, H. Hanada, S. Sasaki, Internal structure of the Moon inferred from Apollo seismic data and selenodetic data from GRAIL and LLR. Geophys. Res. Lett. 42, 7351-7358 (2015). https://doi.org/10.1002/2015GL065335

S. May, Meteor impact detection on Mars with change detection framework, IEEE IGARSS (2018, submitted)

A.S. McEwen et al., Mars Reconnaissance Orbiter's High Resolution Imaging Science Experiment (HiRISE). J. Geophys. Res. 112, E05S02 (2007). https://doi.org/10.1029/2005JE002605

A.S. McEwen, E.M. Eliason, V.C. Gulick, Y. Spinoza, R.A. Beyer (HiRISE Team), HiRISE: The People's Camera. AGU Fall Meeting Abstracts (2010), abstract \#ED23A-0712 
A. McGarr, G.V. Latham, D.E. Gault, Meteoroid impacts as sources of seismicity on the Moon. J. Geophys. Res. 74, 5981-5994 (1969)

H.J. Melosh, Impact Cratering: A Geological Process, Oxford Monographs on Geology and Geophysics (Oxford University Press, New York, 1989)

K. Miljković, G.S. Collins, S. Mannick, P.A. Bland, Morphology and population of binary asteroid impact craters. Earth Planet. Sci. Lett. 363, 121-132 (2013)

K. Miljkovic, E.K. Sansom, I.J. Daubar, F. Karakostas, P. Lognonné, Fate of meteoroid impacts on Mars detectable by the InSight mission, in 47th Lunar Planet. Sci. Conference. LPI Contribution, vol. No (2016), p. 1768

D. Mimoun, N. Murdoch, P. Lognonné, K. Hurst, W.T. Pike, J. Hurley, T. Nébut, W.B. Banerdt, The noise model of the SEIS seismometer of the InSight mission to Mars. Space Sci. Rev. 211, 383-428 (2017). https://doi.org/10.1007/s11214-017-0409-x

Morgan et al., A pre-landing assessment of regolith properties at the InSight landing site. Space Sci. Rev. 214(6), 1-47 (2018). https://doi.org/10.1007/s11214-018-0537-y

S.L. Murchie, R.E. Arvidson, P. Bedini, K. Beisser, J.P. Bibring, J. Bishop, J. Boldt et al., Compact Reconnaissance Imaging Spectrometer for Mars (CRISM) on Mars Reconnaissance Orbiter (MRO). J. Geophys. Res., Planets 112(5), 1-57 (2007). https://doi.org/10.1029/2006JE002682

N. Murdoch, D. Mimoun, R.F. Garcia, W. Rapin, T. Kawamura, P. Lognonné, D. Banfield, W.B. Banerdt, Evaluating the wind-induced mechanical noise on the InSight seismometers. Space Sci. Rev. 211(1-4), 429-455 (2017). https://doi.org/10.1007/s11214-016-0311-y

Y. Nakamura, New identification of deep moonquakes in the Apollo lunar seismic data. Phys. Earth Planet. Inter. 139(3-4), 197-205 (2003). https://doi.org/10.1016/j.pepi.2003.07.017

Y. Nakamura, Timing problem with the lunar module impact data as recorded by the LSPE and corrected near-surface structure at the Apollo 17 landing site. J. Geophys. Res., Planets 116(12), 3-5 (2011). https://doi.org/10.1029/2011JE003972

Y. Nakamura, D.L. Anderson, Martian wind activity detected by a seismometer at Viking lander 2 site. Geophys. Res. Lett. 6, 499-502 (1979). https://doi.org/10.1029/GL006i006p00499

Y. Nakamura, D.R. Lammlein, G.V. Latham et al., New seismic data on the state of the deep lunar interior. Science 181, 49-51 (1973). https://doi.org/10.1126/science.181.4094.49

Y. Nakamura, G.V. Latham, H.J. Dorman, F.K. Duennebier, Seismic structure of the Moon: a summary of current status, in Proc. Lunar Sci. Conf., vol. 7 (1976), pp. 3113-3121

L. Neslusan, J. Svoren, V. Porubcan, A computer program for calculation of a theoretical meteor-stream radiant. Astron. Astrophys. 331, 411-413 (1998)

G. Neukum, B. Ivanov, Crater size distributions and impact probabilities on Earth from lunar, terrestrialplanet, and asteroid cratering data, in Hazards due to Comets and Asteroids, ed. by T. Gehrels, M.S. Matthews, A. Schumann (University of Arizona Press, Tucson, AZ, 1994), pp. 359-416

G. Neukum, R. Jaumann (the HRSC Co-Investigator Team HRSC), The high resolution stereo camera of Mars Express. ESA special publications SP-1240 (2004)

G. Neukum, D.U. Wise, Mars: a standard crater curve and possible new time scale. Science 194(4272), 13811387 (1976)

T. Nissen-Meyer, M. van Driel, S.C. Stähler, K. Hosseini, S. Hempel, L. Auer, A. Colombi, A. Fournier, AxiSEM: broadband 3-D seismic wavefields in axisymmetric media. Solid Earth 5, 425-445 (2014). https://doi.org/10.5194/se-5-425-2014

J. Oberst, Y. Nakamura, A new estimate of the meteoroid impact flux on the Moon, in Proceedings of the Lunar Science Conference XX (1989), pp. 802-803

J. Oberst, A. Christou, R. Suggs, D. Moser, I.J. Daubar, A.S. McEwen, M. Burchell, T. Kawamura, H. Hiesinger, K. Wünnemann, R. Wagner, M.S. Robinson, The present-day flux of large meteoroids on the lunar surface — a synthesis of models and observational techniques. Planet. Space Sci. 74, 179-193 (2012)

M.P. Panning, E. Beucler, M. Drilleau, A. Mocquet, P. Lognonné, W.B. Banerdt, Verifying single-station seismic approaches using Earth-based data: preparation for data return from the InSight mission to Mars. Icarus 248, 230-242 (2015). https://doi.org/10.1016/j.icarus.2014.10.035

M. Panning, P. Lognonné, W.B. Banerdt, R. Garcia, M. Golombek, S. Kedar, B. Knapmeyer-Endrun, A. Mocquet, N.A. Teanby, J. Tromp, R. Weber, E. Beucler, J.-F. Blanchette-Guertin, E. Bozdag, M. Drilleau, T. Gudkova, S. Hempel, A. Khan, V. Lekic, N. Murdoch, A.-C. Plesa, A. Rivoldini, N. Schmerr, Y. Ruan, O. Verhoeven, C. Gao, U. Christensen, J. Clinton, V. Dehant, D. Giardini, D. Mioun, W.T. Pike, S. Smrekar, M. Wieckzorek, M. Knapmeyer, J. Wookey, Planned products of the Mars structure service for the InSight mission to Mars. Space Sci. Rev. 221, 611-650 (2017)

Q.R. Passey, H. Melosh, Effects of atmospheric breakup on crater field formation. Icarus 42(2), 211-233 (1980) 
H.J. Patton, W.R. Walter, Regional moment—magnitude relations for earthquakes and explosions. Geophys. Res. Lett. 20, 277-280 (1993)

J. Peterson, Observations and modeling of seismic background noise. U.S. Geol. Surv. Tech. Rept. 93-322, $1-95$ (1993)

N.A. Petersson et al. Lawrence Livermore National Laboratory Technical Report LLNL-TR-422928 (2010)

A.C. Plesa, M. Grott, N. Tosi, D. Breuer, T. Spohn, M. Wieczorek, How large are present-day heat flux variations across the surface of Mars? J. Geophys. Res. (2016). https://doi.org/10.1002/2016JE005126

A.C. Plesa, M. Knapmeyer, M.P. Golombek, D. Breuer, M. Grott, T. Kawamura, P. Lognonné, N. Tosi, R.C. Weber, Present-day Mars' seismicity predicted from 3-D thermal evolution models of interior dynamics. Geophys. Res. Lett. 45(6), 2580-2589 (2018). https://doi.org/10.1002/2017GL076124

J.B. Plescia, M.S.S. Robinson, R. Wagner, R. Baldridge, Ranger and Apollo S-IVB spacecraft impact craters. Planet. Space Sci. 124 (May). Elsevier, 15-35 (2016). https://doi.org/10.1016/j.pss.2016.01.002

P.W. Pomeroy, Long period seismic waves from large, near-surface nuclear explosions. Bull. Seismol. Soc. Am. 53, 109-149 (1963)

O.P. Popova, I.V. Nemtchinov, W.K. Hartmann, Bolides in the present and past Martian atmosphere and effects on cratering processes. Meteorit. Planet. Sci. 38(6), 905-925 (2003). https://doi.org/10.1111/ j.1945-5100.2003.tb00287.x

O. Popova, J. Borovička, W.K. Hartmann, P. Spurnỳ, E. Gnos, I. Nemtchinov, J.M. Trigo-Rodríguez, Very low strengths of interplanetary meteoroids and small asteroids. Meteorit. Planet. Sci. 46(10), 1525-1550 (2011)

W.L. Quaide, V.R. Oberbeck, Thickness determinations of the lunar surface layer from lunar impact craters. J. Geophys. Res. 73, 5247-5270 (1968)

P.J. Register, D.L. Mathias, L.F. Wheeler, Asteroid fragmentation approaches for modeling atmospheric energy deposition. Icarus 284, 157-166 (2017)

J.E. Richardson, S. Kedar, An experimental investigation of the seismic signal produced by hypervelocity impacts, in Lunar and Planetary Science Conference 44 (2013), abstract 2863

J.E. Richardson, H.J. Melosh, R.J. Greenberg, D.P. O'Brien, The global effects of impact-induced seismic activity on fractured asteroid surface morphology. Icarus 179(2), 325-349 (2005). https://doi.org/ 10.1016/j.icarus.2005.07.005

A. Rivoldini, T. Van Hoolst, O. Verhoeven, A. Mocquet, V. Dehant, Geodesy constraint on the interior structure and composition of Mars. Icarus 213(2), 451-472 (2011). https://doi.org/10.1016/ j.icarus.2011.03.024

S.W. Ruff, P.R. Christensen, Bright and dark regions on Mars: particle size and mineralogical characteristics based on thermal emission spectrometer data. J. Geophys. Res., Planets 107, 1-22 (2002). https://doi.org/10.1029/2001JE001580

N.C. Schmerr, M.E. Banks, I.J. Daubar, The seismic signatures of impact events on Mars: implications for the InSight lander, in LPSC 47 (2016), abstract 1320

R.M. Schmidt, K.R. Housen, Some recent advances in the scaling of impact and explosion cratering. Int. J. Impact Eng. 5, 543-560 (1987). https://doi.org/10.1016/0734-743X(87)90069-8

F. Selsis, M.T. Lemmon, J. Vaubaillon, J.F. Bell III., Extraterrestrial meteors: a Martian meteor and its parent comet. Nature 435, 581 (2005). https://doi.org/10.1038/435581a

L.E. Senft, S.T. Stewart, Modeling impact cratering in layered surfaces. J. Geophys. Res. 112, E11002 (2007). https://doi.org/10.1029/2007JE002894

D.E. Shean, O. Alexandrov, Z.M. Moratto, B.E. Smith, I.R. Joughin, C. Porter, P. Morin, An automated, open-source pipeline for mass production of digital elevation models (DEMs) from very-high-resolution commercial stereo satellite imagery. ISPRS J. Photogramm. Remote Sens. 116, 101 (2016)

P. Shearer, Introduction to Seismology, 2nd edn. (Cambridge University Press, Cambridge, 2009). 412 pp.

N.I. Shishkin, Seismic efficiency of a contact explosion and a high-velocity impact. J. Appl. Mech. Tech. Phys. 48, 145-152 (2007). https://doi.org/10.1007/s10808-007-0019-6

Smrekar et al., Pre-mission InSights on the interior of Mars. Space Sci. Rev. (2018). https://doi.org/10.1007/ s11214-018-0563-9

F. Sohl, T. Spohn, The interior structure of Mars: implications from SNC meteorites. J. Geophys. Res. 102, 1613-1636 (1997). https://doi.org/10.1029/96JE03419

T. Spohn et al. HP3 Instrument Paper. Space Sci. Rev. (2018, this issue)

J. Stevanović, N.A. Teanby, J. Wookey, N. Selby, I.J. Daubar, J. Vaubaillon, R. Garcia, Bolide airbursts as a seismic source for the 2018 Mars InSight mission. Space Sci. Rev. 211, 525-545 (2017)

J. Sweeney, N.H. Warner, M.P. Golombek, R. Kirk, R.L. Fergason, A. Pivarunas, Crater Degradation and Surface Erosion Rates at the InSight Landing Site, Western Elysium Planitia, Mars (Expanded Abstract). 47th Lunar and Planetary Science, Abstract \#1576, Lunar and Planetary (Institute, Houston, 2016)

G. Tancredi, J. Ishitsuka, P.H. Schultz, R.S. Harris, P. Brown, D.O. ReVelle, K. Antier, A. Le Pichon, D. Rosales, E. Vidal, M.E. Varela, L. Sanchez, S. Benavente, J. Bojorquez, D. Cabezas, A. Dalmau, A 
meteorite crater on Earth formed on September 15, 2007: the Carancas hypervelocity impact. Meteorit. Planet. Sci. 44, 1967-1984 (2009)

Teanby, Predicted detection rates of regional-scale meteorite impacts on Mars with the InSight short-period seismometer. Icarus 256, 49-62 (2015)

N.A. Teanby, J. Wookey, Seismic detection of meteorite impacts on Mars. Phys. Earth Planet. Inter. 186, 70-80 (2011)

N. Thomas, G. Cremonese, R. Ziethe, M. Gerber, M. Brändli, G. Bruno, J.J. Wray, The Colour and Stereo Surface Imaging System (CaSSIS) for the ExoMars Trace Gas Orbiter. Space Sci. Rev. 212(3-4), $1897-$ 1944 (2017). https://doi.org/10.1007/s11214-017-0421-1

M.N. Toksöz, F. Press, K. Anderson, A. Dainty, G. Latham, M. Ewing, F. Duennebier, Velocity structure and properties of the lunar crust. Moon 4(3-4), 490-504 (1972). https://doi.org/10.1007/BF00562013

Vaubaillon, A confidence index for forecasting of meteor showers. Planet. Space Sci. 143, 78-82 (2017)

J. Vaubaillon, F. Colas, L. Jorda, A new method to predict meteor showers. I. Description of the model. Astron. Astrophys. 439(2), 751-760 (2005)

R.V. Wagner, D.M. Nelson, J.B. Plescia, M.S. Robinson, E.J. Speyerer, E. Mazarico, Coordinates of anthropogenic features on the Moon. Icarus 283, 92-103 (2017). https://doi.org/10.1016/j.icarus.2016.05.011

J.D. Walker, Loading sources for seismological investigations of asteroids and comets. Int. J. Impact Eng. 29, 757-769 (2003)

N.H. Warner, M.P. Golombek, J. Sweeney, R. Fergason, R. Kirk, C. Schwartz, Near surface stratigraphy and regolith production in southwestern Elysium Planitia, Mars: implications for HesperianAmazonian terrains and the InSight lander mission. Space Sci. Rev. 211, 147-190 (2017). https://doi.org/10.1007/s11214-017-0352-x

W.A. Watters, L.M. Geiger, M. Fendrock, R. Gibson, Morphometry of small recent impact craters on Mars: size and terrain dependence, short-term modification. J. Geophys. Res., Planets 120, 226-254 (2015). https://doi.org/10.1002/2014JE004630

G.C. Werth, R.F. Herbst, Comparison of amplitudes of seismic waves from nuclear explosions in four mediums. J. Geophys. Res. 68, 1463-1475 (1963). https://doi.org/10.1029/JZ068i005p01463

L.F. Wheeler, P.J. Register, D.L. Mathias, A fragment-cloud model for asteroid breakup and atmospheric energy deposition. Icarus 295, 149-169 (2017)

F.J.W. Whipple, The great Siberian meteor and the waves, seismic and aerial, which it produced. Q. J. R. Meteorol. Soc. 56, 287-304 (1930)

E.A. Whitaker, in Artificial Lunar Impact Craters: Four New Identifications. NASA Special Publication, vol. 315 (1972), pp. 29-39

J.-P. Williams, Acoustic environment of the Martian surface. J. Geophys. Res., Planets 106(E3), 5033-5041 (2001)

J.-P. Williams, A.V. Pathare, O. Aharonson, The production of small primary craters on Mars and the Moon. Icarus 235, 23-36 (2014)

J.H. Woodhouse, The calculation of eigenfrequencies and eigenfunctions of the free oscillations of the Earth and the Sun, in Seismological Algorithms, Computational Methods and Computer Programs, ed. by D.J. Doornbos (Academic Press, London, 1988), pp. 321-370

K. Wunnemann, D. Nowka, G.S. Collins, D. Elbeshausen, M. Bierhaus, Scaling of impact crater formation on planetary surfaces-insights from numerical modeling, in Proc. 11th Hypervelocity Impact Symposium, no. 120 (2011)

M. Yasui, E. Matsumoto, M. Arakawa, Experimental study on impact-induced seismic wave propagation through granular materials. Icarus 260, 320-331 (2015) 